\author{
Monograph \\ urn:1sid:zoobank.org:pub:3FFC527D-E9CA-4D9F-BF59-076FA60171AC
}

\title{
Revision of the land snail genus Landouria Godwin-Austen, 1918 (Gastropoda, Camaenidae) from Java
}

\author{
Ayu Savitri NURINSIYAH ${ }^{1}$, Marco T. NEIBER ${ }^{2}$ \& Bernhard HAUSDORF ${ }^{3, *}$ \\ ${ }^{1.2,3}$ Zoological Museum, Center of Natural History, University of Hamburg, \\ Martin-Luther-King-Platz 3, 20146 Hamburg, Germany. \\ ${ }^{1}$ Current address: Museum Zoologicum Bogoriense, Research Center for Biology, \\ Indonesian Institute of Science, Cibinong, West Java, Indonesia. \\ *Corresponding author: hausdorf@zoologie.uni-hamburg.de \\ ${ }^{1}$ Email: ayu_nurinsiyah@yahoo.com; ayus002@lipi.go.id \\ 2Email: mneiber@hotmail.de \\ ${ }^{1}$ urn:lsid:zoobank.org:author:5AFBFEFB-B243-49CC-AACC-248B2F007BC8 \\ ${ }^{2}$ urn:1sid:zoobank.org:author:8F6006F5-D880-4841-AC32-B97E18D7BB5C \\ ${ }^{3}$ urn:Isid:zoobank.org:author:FEDE92C0-108D-4315-8F86-9F2CFF1CB906
}

\begin{abstract}
A revision of the land snail genus Landouria Godwin-Austin, 1918 (Camaenidae) from Java reveals that this group represents the most diverse land snail radiation on that island. Only six species of Landouria were recognized from Java in the last revision of the genus based on shell characters. Our investigation, which also considers the genitalia as well as DNA sequences, shows that the diversity in Java is much higher. Based on newly collected specimens as well as museum material, twenty-eight species of Landouria from Java are described and figured. To stabilize the nomenclature, neotypes are designated for L. winteriana (Pfeiffer, 1842) and L. rotatoria (Pfeiffer, 1842). Sixteen species are described as new to science, i.e., L. naggsi sp. nov., L. parahyangensis sp. nov., L. nusakambangensis sp. nov., L. petrukensis sp. nov., L. tholiformis sp. nov., L. madurensis sp. nov., L. abdidalem sp. nov., L. sewuensis sp. nov., L. tonywhitteni sp. nov., L. sukoliloensis sp. nov., L. nodifera sp. nov., L. pacitanensis sp. nov., L. zonifera sp. nov., L. pakidulan sp. nov., L. dharmai sp. nov. and L. menorehensis sp. nov. Landouria conoidea (Leschke, 1914) comb. nov., L. intumescens (Martens, 1867) comb. nov., L. moussoniana (Martens, 1867) comb. nov., L. schepmani (Möllendorff, 1897) comb. nov. and L. leucochila (Gude, 1905) comb. nov. are considered valid species of the genus Landouria for the first time. Plectotropis kraepelini Leschke, 1914 syn. nov. is considered a probable synonym of $L$. winteriana (Pfeiffer, 1842), P. trichotrochium Möllendorff, 1897 syn. nov. is a synonym of L. epiplatia (Möllendorff, 1897) and the preoccupied name Helix squamulosa Martens, 1867 syn. nov. is a synonym of L. madurensis sp. nov. We estimate that there are actually more than fifty species of Landouria in Java because many shell samples could not be classified and because no material is available from several regions of the island. A molecular phylogeny reveals that the species from Java do not form a monophyletic group, but that at least one species from Timor is nested within Javanese clades. This means that the Oriental Landouria crossed Wallace's line, the supposed border between the Oriental and Australo-Papuan regions, at least twice and supports the conclusion that Wallace's line does not represent a more severe barrier for terrestrial organisms than other straits through the archipelago. Within the Javanese clades, species from western and eastern Java are mixed, indicating frequent dispersals also within Java.
\end{abstract}


Keywords. Camaenidae, Indonesia, Java, Landouria, phylogeny.

Nurinsiyah A.S., Neiber M.T. \& Hausdorf B. 2019. Revision of the land snail genus Landouria Godwin-Austen, 1918 (Gastropoda: Camaenidae) from Java. European Journal of Taxonomy 526: 1-73.

https://doi.org/10.5852/ejt.2019.526

\section{Introduction}

The Aegistini Kuroda \& Habe, 1949 (Bradybaeninae Pilsbry, 1934 (1898), Camaenidae Pilsbry, 1895, in the sense of Bouchet et al. 2017, corresponding to Aegista Albers, 1850 in the sense of Hirano et al. 2014) are a highly diverse group of land snails, which is mainly distributed in East and Southeast Asia (Schileyko 2004; Hirano et al. 2014). In the Indo-Australian Archipelago, they are represented by species with a depressed, often angular or keeled, usually brownish-corneous shell with a wide umbilicus. Some of these species were placed in the East Asian group Plectotropis Martens, 1860 by Martens (1867). However, Wiegmann (1893) could not find a dart apparatus in two juvenile specimens of two species from Sumatra and Java, whereas a dart apparatus is present in the East Asian representatives of Plectotropis (Pilsbry 1895 in 1893-1895; Schileyko 2004). Rensch (1932) confirmed the lack of the dart apparatus in adult specimens from the Sunda Islands and suggested the transfer of species from the Indo-Australian Archipelago to Landouria Godwin-Austen, 1918. Landouria was established for species without a dart apparatus from the Himalayas, eastern India, Myanmar and Sri Lanka by GodwinAusten (1918). Schileyko \& Kuznetsov (1998) recognized that Landouria belongs to the Aegistinae Kuroda \& Habe, 1949 despite the lack of the dart apparatus. They described additional species from the Himalayas and questioned the placement of the species from the Indo-Australian Archipelago in Landouria. However, Schileyko (2004) continued to include them with a question mark in Landouria. Páll-Gergely et al. (2013) described an additional species from southern China and questioned the placement of L. radleyi (Jousseaume, 1894) from Sri Lanka in Landouria. The molecular phylogeny of Hirano et al. (2014) showed that Landouria belongs to the Aegistini and is the sister taxon of a group of high-spired species from the Ryukyu Islands and Taiwan, which were formerly erroneously included in the Chinese Pseudobuliminus Gredler, 1887 and which also lack a dart apparatus. Thus, the loss of the dart apparatus is probably a synapomorphy of this group and Landouria. However, Hirano et al. (2014) also showed that the dart apparatus was lost independently at least three times in the Aegistini. Hirano et al. (2014) suggested tentatively to include all species of the Aegistini in a single genus Aegista. This genus would include approximately 200 species distributed from Korea and Japan to India, Nepal and Indonesia, and would display a high disparity with regard to shell and genital morphology. We prefer to split this complex into smaller taxa to facilitate discussions of biogeography, character evolution and other aspects. Thus, we continue the use of the name Landouria for the clade of South Asian species with a depressed shell and without a dart apparatus. This opinion is shared by Köhler et al. (in press), who revised the species from Timor-Leste.

The earliest records of Landouria in Java date back to Pfeiffer (1842). Sixteen nominal species presumably belonging to Landouria had been described from Java until 1950. However, van Benthem Jutting (1950) considered only six of them valid in her revision based on shell characters. One additional species from Java has recently been described by Dharma (2015).

The study of recently collected material proved that many samples could not be unambiguously assigned to the species delimited by van Benthem Jutting (1950) based on shell characters. The anatomy of most of these species has remained unknown. Thus, we started a revision of the genus Landouria from Java by applying an integrative approach combining shell morphology, characters of the genitalia and DNA sequences. Our work revealed the most diverse land snail radiation on Java. We describe or redescribe 28 species here, but we consider it possible that there are about twice as many species of Landouria on Java. 


\section{Material and methods}

\section{Material, morphology and geographic data}

This study is based on material from surveys by the first author in Java and surrounding islands in 2013-2015 as well as museum collections. Most living specimens were killed and preserved in alcohol without extracting the soft body from the shell. We usually made a small hole in the umbilical side at the beginning of the last whorl to sever the columellar muscle, so that at least the anterior part of the body with the taxonomically important distal genitalia could be extracted while keeping shell damage to a minimum.

The material is kept in the following institutions:

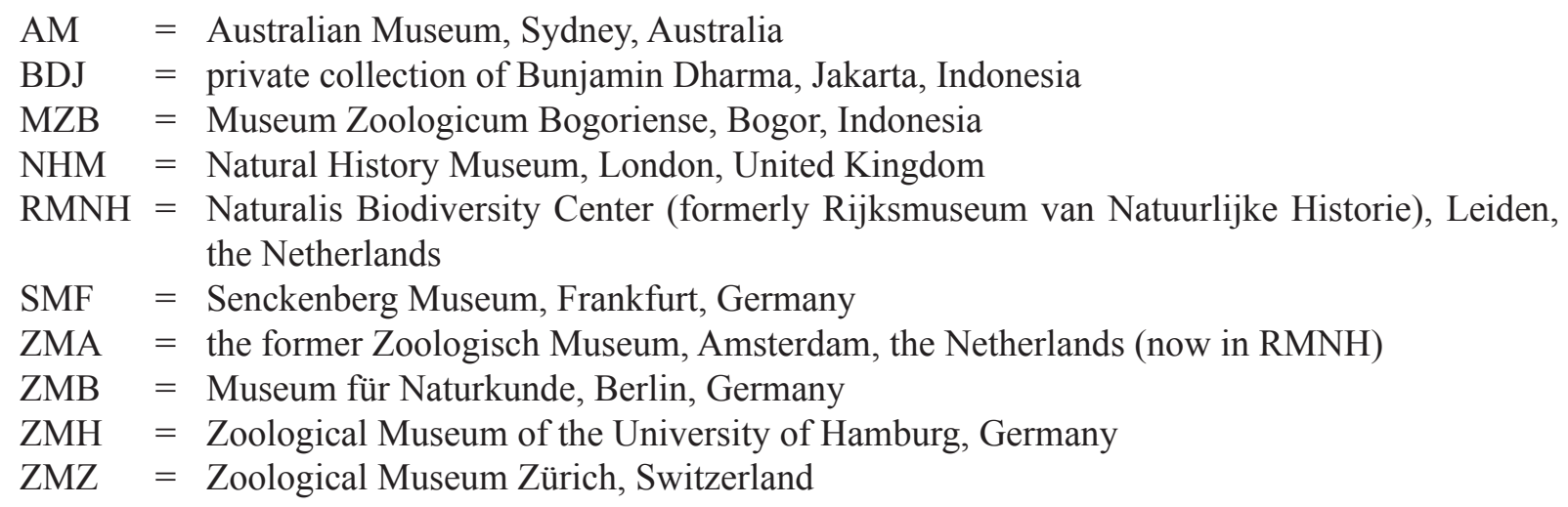

We measured the diameter (D) and height $(\mathrm{H})$ of the shell, the diameter of the third whorl (D3W), the diameter of the inner edge of the umbilicus at the largest diameter of the shell (U), and the diameter (da) and height (ha) of the aperture with a digital calliper or an ocular micrometer (accurate to $0.1 \mathrm{~mm}$ ) and counted the number of whorls with an accuracy of 0.25 whorls following the method described by Kerney \& Cameron (1979). In the descriptions of the genitals, the terms proximal and distal refer to the position in relation to the gonad. Stacked images of shells were taken with the Passport Imaging System (Dun, Palmyra, VA, USA) with a Canon EOS 6D camera (Canon Inc., Tokyo, Japan) and processed with Helicon Focus (HeliconSoft, Kharkiv, Ukraine) and Adobe Photoshop (Adobe Systems Inc., San José, CA, USA) software.

In the lists of material examined, localities are ordered in provinces and listed from west to east. We specify for each locality whether we examined the genitalia of one or several specimens from that locality (det. anat. = anatomically determined). For newly designated name-bearing types, we specify the collector (leg. = collected by).

The International Code of Zoological Nomenclature (International Commission on Zoological Nomenclature 1999) is abbreviated as ICZN.

\section{DNA extraction, amplification and sequencing}

Total genomic DNA was extracted from the foot muscle tissue of recently collected specimens stored in 70\% ethanol (for voucher numbers see Table 1) using a slightly modified version of the protocol of Sokolov (2000) as described in Scheel \& Hausdorf (2012).

Fragments of the mitochondrial 16S rDNA were amplified by polymerase chain reaction (PCR) using the primer pair 16Scs1 (Chiba 1999) plus 16S_MN3R (Neiber et al. 2017). If the PCR with this primer combination was not successful, newly designed internal forward and reverse primers were used in combination with one of the above listed primers: 16S_BF1 (5'-CCA TCR AAA AGT ATY WAG TAC-3') 
Table 1 (continued on next page). Museum catalogue numbers, DNA voucher numbers and GenBank accession numbers for the specimens and DNA sequences used in the molecular phylogenetic analyses. All localities in Java unless otherwise specified.

\begin{tabular}{|c|c|c|c|c|}
\hline Taxon & Locality & $\begin{array}{c}\text { Museum } \\
\text { number }\end{array}$ & DNA voucher & $\begin{array}{c}\text { GenBank accession } \\
\text { number (16S rDNA) }\end{array}$ \\
\hline $\begin{array}{l}\text { L. abdidalem } \\
\text { sp. nov. }\end{array}$ & $\begin{array}{l}\text { Kulonprogo, Sibolong, near } \\
\text { Kiskendo Cave (holotype) }\end{array}$ & MZB 21271 & ZMH Extr 3928 & MK608364 \\
\hline L. ciliocincta & Malang, Kondangmerak & MZB 19211 & AYLAN16 & MK608365 \\
\hline L. epiplatia & Bogor, Gunung Gede & ZMH 79946 & AYLAN45 & MK608366 \\
\hline L. intumescens & $\begin{array}{c}\text { Tuban, Montong, Guwoterus, } \\
\text { sumber Krawak }\end{array}$ & MZB 19207 & AYLAN33 & MK608367 \\
\hline $\begin{array}{l}\text { L. madurensis } \\
\text { sp. nov. }\end{array}$ & $\begin{array}{l}\text { Madura Island, Sampang } \\
\text { (holotype) }\end{array}$ & MZB 21432 & AYLAN17 & MK608368 \\
\hline $\begin{array}{l}\text { L. menorehensis } \\
\text { sp. nov. }\end{array}$ & $\begin{array}{l}\text { Kulonprogo, Sibolong } \\
\text { (holotype) }\end{array}$ & MZB 19201 & AYLAN12 & MK608369 \\
\hline L. montana & $\begin{array}{l}\text { Timor-Leste, Tirsfu near } \\
\text { Kampung Qeurema }\end{array}$ & AM C477070 & - & MH521075 \\
\hline L. montana & $\begin{array}{l}\text { Timor-Leste, Mundo Perdido } \\
\text { Protected Area, Mt Laritame }\end{array}$ & AM C477047 & - & MH521080 ${ }^{1}$ \\
\hline $\begin{array}{l}\text { L. nodifera } \\
\text { sp. nov. }\end{array}$ & $\begin{array}{l}\text { Pati, Larangan Village } \\
\text { (paratype) }\end{array}$ & MZB 21266 & AYLAN8 & MK608370 \\
\hline L. omphalostoma & $\begin{array}{l}\text { China, Yunnan, Xiangyun } \\
\text { Xian, entrance of the } \\
\text { Qinghuadong }\end{array}$ & HNHM 98758 & PGB-a & MH521068 ${ }^{1}$ \\
\hline L. omphalostoma & $\begin{array}{l}\text { China, Yunnan, Xiangyun } \\
\text { Xian, entrance of the } \\
\text { Qinghuadong }\end{array}$ & HNHM 98758 & PGB-b & MH521069 ${ }^{1}$ \\
\hline $\begin{array}{l}\text { L. pacitanensis } \\
\text { sp. nov. }\end{array}$ & $\begin{array}{l}\text { Pacitan, Srau Beach } \\
\text { (holotype) }\end{array}$ & MZB 21191 & AYLAN35 & MK608371 \\
\hline L. rotatoria & Bogor, Blok kancil (neotype) & MZB 19205 & AYLAN39 & MK608372 \\
\hline $\begin{array}{l}\text { L. sewuensis } \\
\text { sp. nov. }\end{array}$ & $\begin{array}{l}\text { Gunung Kidul, Tepus, near } \\
\text { Poktunggal Beach (paratype) }\end{array}$ & MZB 21269 & AYLAN6a & MK608373 \\
\hline $\begin{array}{l}\text { L. sewuensis } \\
\text { sp. nov. }\end{array}$ & $\begin{array}{c}\text { Gunung Kidul, Tepus, Mbajo } \\
\text { Hill (holotype) }\end{array}$ & MZB 19255 & AYLAN42 & MK608374 \\
\hline $\begin{array}{l}\text { L. sewuensis } \\
\text { sp. nov. }\end{array}$ & $\begin{array}{c}\text { Gunung Kidul, Tepus, Mbajo } \\
\text { Hill (paratype) }\end{array}$ & MZB 19256 & AYLAN5 & MK608375 \\
\hline $\begin{array}{l}\text { L. sewuensis } \\
\text { sp. nov. }\end{array}$ & $\begin{array}{l}\text { Gunung Kidul, Tepus, near } \\
\text { Poktunggal Beach (paratype) }\end{array}$ & ZMH 79891 & - & MK613237 \\
\hline $\begin{array}{l}\text { L. sukoliloensis } \\
\text { sp. nov. }\end{array}$ & $\begin{array}{l}\text { Pati, Kayen, near Watu } \\
\text { Payung (holotype) }\end{array}$ & MZB 19219 & ZMH Extr 3931 & MK613236 \\
\hline $\begin{array}{l}\text { L. sukoliloensis } \\
\text { sp. nov. }\end{array}$ & $\begin{array}{l}\text { Pati, Sukolilo, Kedumulyo } \\
\text { (paratype) }\end{array}$ & MZB 19222 & AYLAN37 & MK608376 \\
\hline $\begin{array}{l}\text { L. tholiformis } \\
\text { sp. nov. }\end{array}$ & $\begin{array}{l}\text { Gunung Kidul, Tepus, near } \\
\text { Poktunggal Beach (holotype) }\end{array}$ & MZB 17999 & AYLAN14 & MK608377 \\
\hline L. timorensis & $\begin{array}{l}\text { Timor-Leste, Atauro Island, } \\
1.5 \mathrm{~km} \text { N of Beloi (paratype) }\end{array}$ & AM C468777a & - & MH521081' \\
\hline
\end{tabular}

${ }^{1}$ Köhler et al. (in press). 
Table 1 (continued).

\begin{tabular}{|c|c|c|c|c|}
\hline Taxon & Locality & $\begin{array}{c}\text { Museum } \\
\text { number }\end{array}$ & DNA voucher & $\begin{array}{c}\text { GenBank accession } \\
\text { number (16S rDNA) }\end{array}$ \\
\hline L. timorensis & $\begin{array}{l}\text { Timor-Leste, Atauro Island, } \\
\text { Villa, } 1.8 \mathrm{~km} \text { WSW of Tua } \\
\text { Coin Eco Village }\end{array}$ & AM C470449 & - & MH521079 ${ }^{1}$ \\
\hline $\begin{array}{l}\text { L. tonywhitteni } \\
\text { sp. nov. }\end{array}$ & $\begin{array}{l}\text { Grobogan, Taruman } \\
\text { (holotype) }\end{array}$ & MZB 19214 & AYLAN50 & MK608378 \\
\hline $\begin{array}{l}\text { L. tonywhitteni } \\
\text { sp. nov. }\end{array}$ & $\begin{array}{l}\text { Grobogan, Taruman } \\
\text { (paratype) }\end{array}$ & MZB 21268 & AYLAN28 & MK608379 \\
\hline L. winteriana & Bogor, Cisarua & MZB 19235 & AYLAN21 & MK608380 \\
\hline L. winteriana & Padalarang, Gunung Pawon & MZB 19236 & AYLAN24 & MK608381 \\
\hline $\begin{array}{l}\text { L. zonifera } \\
\text { sp. nov. }\end{array}$ & $\begin{array}{l}\text { Kulonprogo, Sibolong } \\
\text { (holotype) }\end{array}$ & MZB 17727 & AYLAN47 & MK608382 \\
\hline $\begin{array}{l}\text { L. zonifera } \\
\text { sp. nov. }\end{array}$ & Kulonprogo, Jonggrangan & ZMH 140031 & ZMH Extr 3929 & MK608383 \\
\hline$L . \mathrm{sp}$. & $\begin{array}{c}\text { Timor-Leste, Manatuto } \\
\text { District, } 4.7 \mathrm{~km} \text { E of Laclubar }\end{array}$ & AM C477078 & - & MH521073' \\
\hline $\begin{array}{l}\text { Mandarina } \\
\text { suenoae }\end{array}$ & $\begin{array}{l}\text { Japan, Chichijima } \\
\text { Archipelago, Anijima }\end{array}$ & - & - & AY829638² \\
\hline $\begin{array}{l}\text { Euhadra } \\
\text { peliomphala }\end{array}$ & Japan, Izu Peninsula, Nagaoka & - & - & AF $104053^{3}$ \\
\hline
\end{tabular}

${ }^{1}$ Köhler et al. (in press); ${ }^{2}$ Davison \& Chiba (2006); ${ }^{3}$ Hayashi \& Chiba (2000).

plus 16S_MN3R, 16Scs1 plus 16S_BR1 (5'-CAA AGC CTA RAC ACY GYG CA-3') or 16Scs1 plus 16S_BR2 (5'-CAA AGC CTG GCC ACT RYG CA-3').

Amplifications were performed in $25 \mu \mathrm{L}$ volumes containing $18.3 \mu \mathrm{L} \mathrm{ddH}_{2} \mathrm{O}, 2.5 \mu \mathrm{L}$ Dream Taq ${ }^{\mathrm{TM}}$ Green Buffer (Thermo Fisher Scientific, Waltham, MA, USA), $1.0 \mu \mathrm{L}$ of a dNTP mix ( $5 \mathrm{mM}$ each), $1.0 \mu \mathrm{L}$ of each primer $(10 \mu \mathrm{M}), 0.2 \mu \mathrm{L}$ DreamTaq ${ }^{\mathrm{TM}}$ DNA polymerase (Thermo Fisher Scientific) and $1.0 \mu \mathrm{L}$ of the template DNA under the following reaction conditions: an initial denaturation step at $94{ }^{\circ} \mathrm{C}$ for $2 \mathrm{~min}, 35-45 \mathrm{PCR}$ cycles $\left(94{ }^{\circ} \mathrm{C}\right.$ for $30 \mathrm{~s}, 50{ }^{\circ} \mathrm{C}$ for $30 \mathrm{~s}, 72{ }^{\circ} \mathrm{C}$ for $\left.30 \mathrm{~s}\right)$ and a final extension step at $72^{\circ} \mathrm{C}$ for $5 \mathrm{~min}$. Both strands of the amplified products were sequenced at Macrogen Europe Laboratory (Amsterdam, the Netherlands).

\section{Phylogenetic analyses of DNA sequence data}

Forward and reverse sequences were assembled using ChromasPro 1.7.4 (Technelysium, Tewantin, QLD, Australia). Obtained sequences were deposited in GenBank (for accession numbers see Table 1). Additionally, we included sequences from species of Landouria from the study of Köhler et al. (in press) as well as sequences of Mandarina suenoae Minato, 1978 (Davison \& Chiba 2006) and Euhadra peliomphala (Pfeiffer, 1850) (Hayashi \& Chiba 2000) as outgroups (see Table 1). The 16S rDNA sequences were aligned with MAFFT 7 (Katoh et al. 2017), using the Q-INS-i strategy and otherwise default settings.

An appropriate evolutionary model was selected with PartitionFinder 2.1.1 (Lanfear et al. 2016) conducting an exhaustive search, with the Bayesian information criterion to select among models and partitions. The models were limited to those available in MrBayes 3.2.6 (Ronquist et al. 2012) as well as those in GARLI 2.1 (Zwickl 2006). The PartitionFinder analysis suggested the HKY $+\mathrm{I}+\mathrm{G}$ model. 
Bayesian inference (BI) and maximum likelihood (ML) analyses were used to reconstruct phylogenetic relationships. The BI analysis was performed using MrBayes 3.2.6. Metropolis-coupled Monte Carlo Markov chain $\left(\mathrm{MC}^{3}\right)$ searches were run with four chains in two separate runs, with 50000000 generations with default priors, trees sampled every 1000 generations under default heating using the evolutionary model as suggested by the PartitionFinder analyses. The first 50000 generations of each run were discarded as a burn-in.

The ML analysis was performed using GARLI ver. 2.1 (http://www.bio.utexas.edu/faculty/antisense/ garli/Garli.html), with the evolutionary model as suggested by PartitionFinder and otherwise default settings from the standard configuration file. Support values were calculated by bootstrapping with 1000 replications.

Bootstrap support (BS) values from the ML analysis, as well as posterior probabilities (PP) from the BI analysis, were mapped on the BI 50\% majority rule consensus tree with SumTrees ver. 3.3.1, which is part of the DendroPy ver. 3.8.0 package (Sukumaran \& Holder 2010). PP $\geq 0.95$ and $B S \geq 70$ were interpreted as positive support for nodes.

\section{Results}

\section{Molecular phylogeny}

The aligned 16S rDNA fragments had a length of 526 base pairs. The p-distances between 16S rDNA sequences of different species of Landouria from Java and Madura Island vary between 0.026 and 0.310 (Table 2) and reach up to 0.384 between the Indonesian species and L. omphalostoma Páll-Gergely \& Hunyadi, 2013 from China. The high p-distances between the morphologically delimited species from Java (Table 2) support the conclusion that these populations really represent distinct species, even though some differ only in a few characters of the genitalia.

In the ML and $\mathrm{BI}$ analyses of the $16 \mathrm{~S}$ rDNA sequences, $L$. omphalostoma from China represents the sister group of the Indonesian species of Landouria (Fig. 1). The Javanese species that are represented by several specimens form monophyletic groups in the $16 \mathrm{~S}$ rDNA tree (Fig. 1). However, the species of Landouria from Java do not form a monophyletic group. At least Landouria sp. nov. (= L. winteriana (not Pfeiffer, 1842) in Köhler et al. in press) from Timor is nested within Javanese clades. The relationships of L. timorensis ${ }^{*}$ Köhler et al., in press and L. montana* Köhler et al., in press from Timor and the Javanese clades are not resolved and it cannot be excluded that these two species from Timor form a clade. Nevertheless, Timor has been colonized at least twice. This means that the Oriental Landouria crossed Wallace's line, the supposed border between the Oriental and Australo-Papuan regions, at least twice. This supports the conclusion that Wallace's line does not represent a more severe barrier for terrestrial organisms than other straits through the archipelago (Hausdorf 2019). Within the Javanese clades, species from western and eastern Java are mixed, indicating frequent dispersals also within Java.

The three species that are characterized by the unusual node at the flagellum and/or a distinct penis coecum, L. tonywhitteni sp. nov., L. nodifera sp. nov. and L. pacitanensis sp. nov. form a clade. However, it remains unclear whether the node at the flagellum is a synapomorphy of $L$. tonywhitteni sp. nov. and L. nodifera sp. nov. (or was lost in L. pacitanensis sp. nov.) and whether the penis coecum is a synapomorphy of L. nodifera sp. nov. and L. pacitanensis sp. nov. (or was lost in L. tonywhitteni sp. nov.), because the relationships among these three species were not resolved.

\footnotetext{
* Note that the names Landouria timorensis and L. montana were not made available by the preliminary online version of Köhler et al. (in press) according to ICZN Art. 8.5.3, 9.9 and 21.8.3, and will only become available when the print version is published.
} 


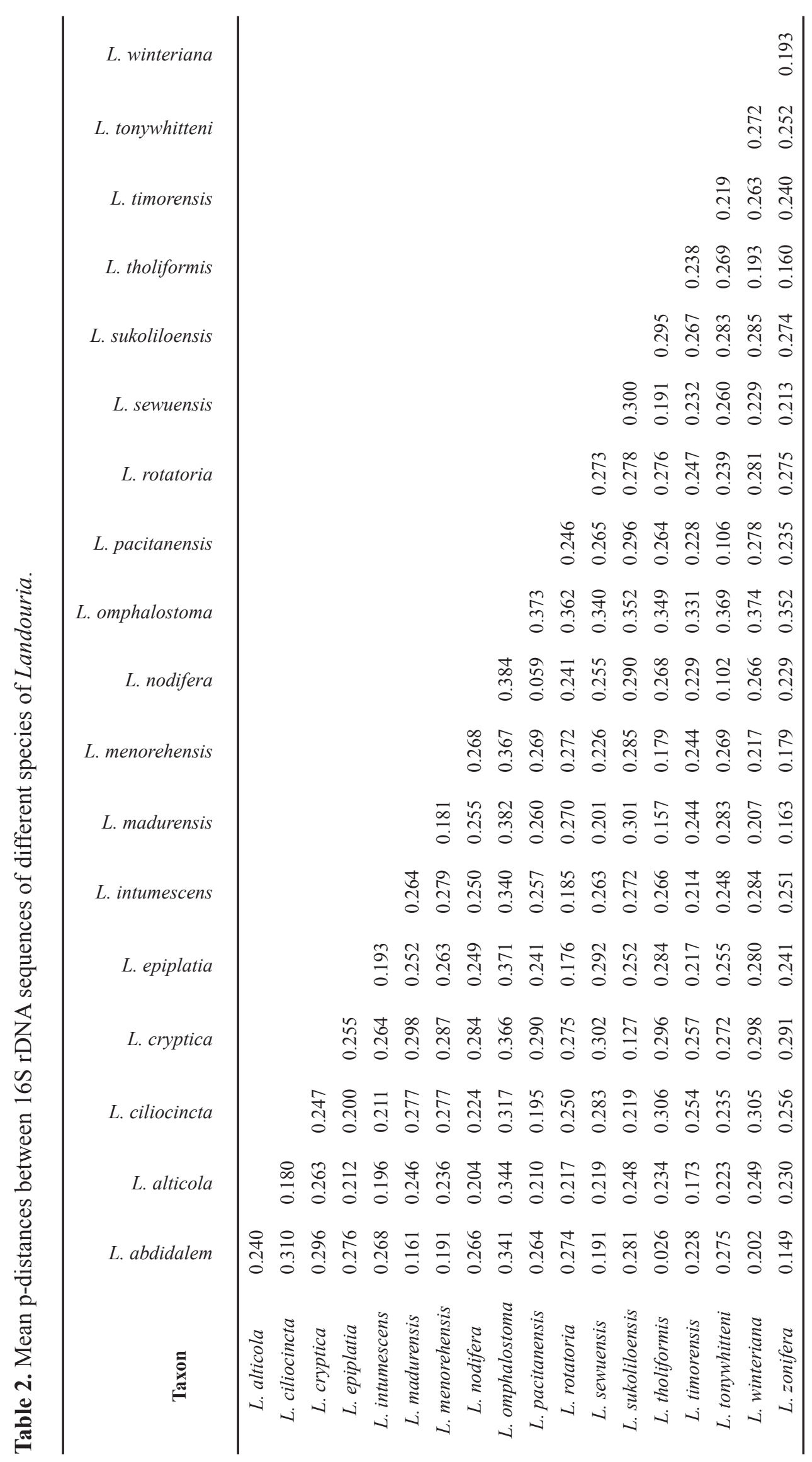




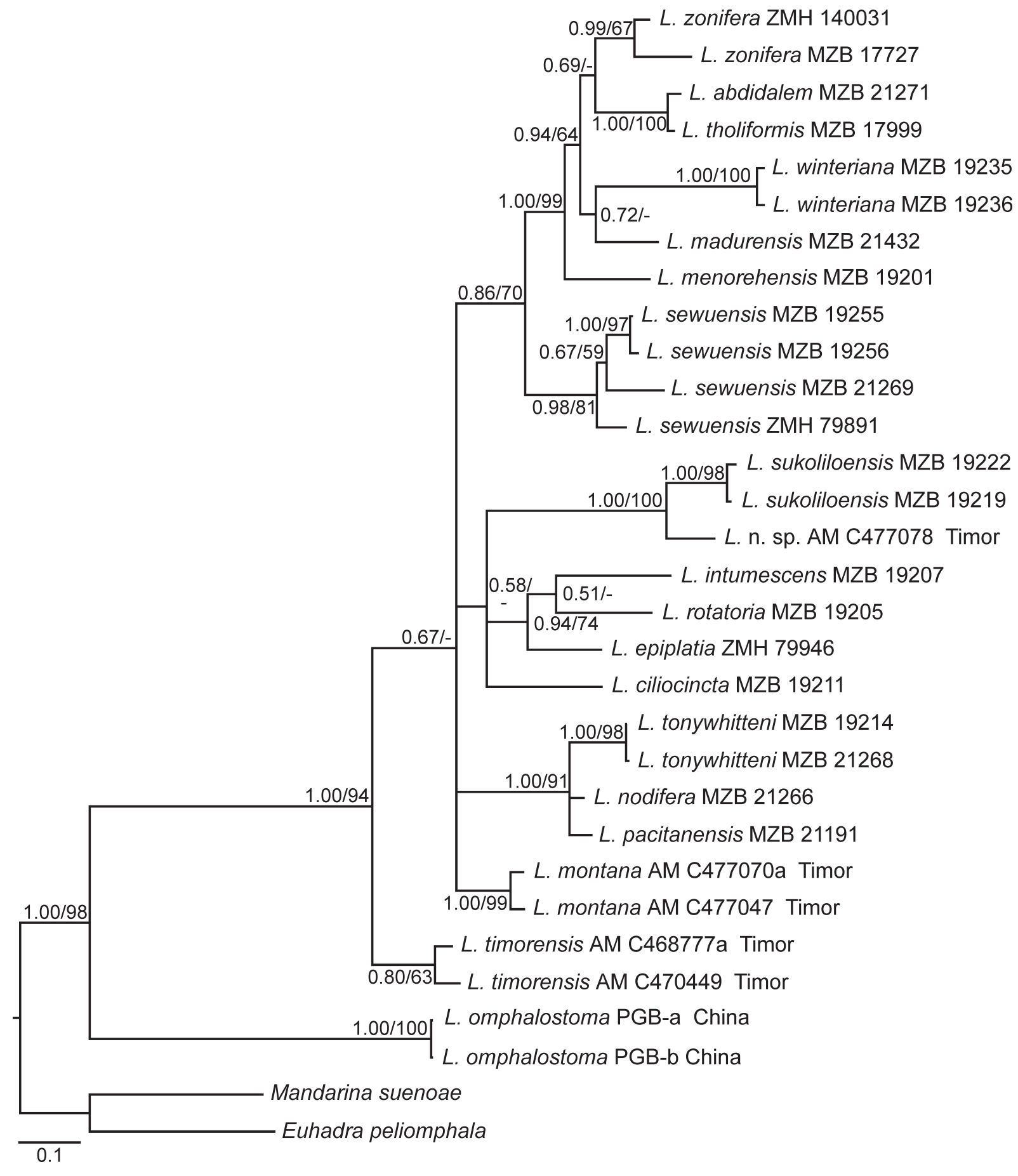

Fig. 1. Bayesian 50\% majority rule consensus tree based on mitochondrial $16 \mathrm{~S}$ rDNA sequences of species of Landouria Godwin-Austen, 1918 with Mandarina suenoae Minato, 1978 and Euhadra peliomphala (Pfeiffer, 1850) as outgroups. All species of Landouria in this tree are from Java except L. omphalostoma Páll-Gergely \& Hunyadi, 2013 from China and L. timorensis Köhler et al. in press, L. montana Köhler et al. in press and Landouria sp. nov. (= L. winteriana (non Pfeiffer, 1842) in Köhler et al. (in press)) from Timor. Values at the nodes represent Bayesian posterior probabilities (left) and maximum likelihood bootstrap values (right). Numbers after taxon names correspond to MZB and ZMH catalogue numbers, AM catalogue numbers or GenBank accession numbers (see Table 1). 
Landouria ciliocincta (Möllendorff, 1897) (Figs 83-84) and L. menorehensis sp. nov. (Fig. 88) share a whitish basic coloration of the shell, whereas the basic coloration of the shell is brownish-corneous in most species of Landouria. The molecular phylogeny indicates that the species with a whitish basic coloration of the shell do not form a clade and, thus, that the transition from a brownish-corneous to a whitish basic coloration of the shell occurred convergently in different clades of Landouria. Whereas the species with a brownish-corneous basic coloration of the shell usually live among debris on the forest floor, L. menorehensis sp. nov. lives on banana trees and on the leaves of snakefruit trees and the whitish basic coloration of the shell might be an adaptation to an arboreal life style.

\section{Systematics}

Family Camaenidae Pilsbry, 1895

Subfamily Bradybaeninae Pilsbry, 1934 (1898)

Tribe Aegistini Kuroda \& Habe, 1949

Genus Landouria Godwin-Austen, 1918

Landouria Godwin-Austen, 1918: 604 (type species by original designation: Helix huttonii Pfeiffer, 1842 (new name for Helix orbicula Hutton \& Benson, 1838, not H. orbicula Orbigny, 1835)).

Thaitropis Schileyko, 2004: 1632, fig. 2104 (type species by original designation: Helix goniochila Pfeiffer, 1862 (= Helix ptychostyla Martens, 1860; see Schileyko 2011)).

\section{Remarks}

Schileyko \& Kuznetsov (1998) pointed out that the features of Landouria correspond to those of Aegista with the exception of the lack of a dart apparatus. Furthermore, they supposed that the dart apparatus might have been lost several times within the Aegistini, once in the Indian lineage and once in the lineage of the species from the Indo-Australian Archipelago and the Philippines. They argued that this hypothesis is also supported by the different structure of the flagellum, which bears external tubercles in the continental lineage but is indented in the Archipelagic lineage. The molecular phylogeny of Hirano et al. (2014) showed that the dart apparatus was actually lost at least three times within the Aegistini and other studies have shown that the loss of the dart apparatus is also a common homoplasy in other groups of the Helicoidea (Neubert 1998, 2014; Wade et al. 2007; Neiber \& Hausdorf 2015; Neiber et al. 2017). However, the molecular phylogeny of Köhler et al. (in press) showed that the species of Landouria from the Sunda Islands form a clade with L. omphalostoma Páll-Gergely \& Hunyadi, 2013 from China. The structure of the flagellum is variable in the species from the Asian mainland (Schileyko \& Kuznetsov 1998; Páll-Gergely et al. 2013) as well as in those from the Indo-Australian Archipelago (see Köhler et al. in press and results below). Thus, there is no reason to assume an independent loss of the dart apparatus in the continental and Archipelagic lineages.

Hirano et al. (2014) showed that Landouria is the sister taxon of a group of high-spired species from the Ryukyu Islands and Taiwan, which were formerly erroneously included in the Chinese Pseudobuliminus Gredler, 1887, which also lack a dart apparatus. Thus, the loss of the dart apparatus is probably a synapomorphy of this group and Landouria. Landouria differs from this group in the depressed conical or globular shell, which usually has periostracal scales or hairs. However, these character states are plesiomorphies that Landouria shares, e.g., with Aegista. Currently, no morphological apomorphies of Landouria are known.

Schileyko (2004) separated two species from Thailand in a distinct genus, Thaitropis. He considered a sharp constriction between the epiphallus and penis as the main diagnostic character of Thaitropis. We also found a similar constriction in L. pacitanensis sp. nov. and in a less drastic form in L. intumescens 
(Martens, 1867) and L. smimensis (Mousson, 1848) from Java. There is a verge in the dilatation proximal of the constriction in L. pacitanensis sp. nov. Thus, the constriction is not situated between the epiphallus and penis, but within the penis. Schileyko did not find a verge, but there is also a small dilatation proximal of the constriction in the Thai species figured by Schileyko. Thus, we suppose that the constriction is also within the penis in the Thai species. As long as the phylogenetic relationships within Landouria are not better resolved and the genus is not further subdivided, we consider Thaitropis to be a synonym of Landouria. However, it is possible that the Southeast Asian species and the Himalayan species, to which the type species of Landouria, L. huttonii (Pfeiffer, 1842), belongs, form separate clades that might be classified at least as subgenera. In this case the name Thaitropis would be available for the Southeast Asian clade.

In the following, the species are ordered according to similarities to facilitate comparisons.

Landouria winteriana (Pfeiffer, 1842)

Figs 2-3, 15, 29, 52; Tables 3-4

Helix winteriana Pfeiffer, 1842: 41 (“Java").

?Plectotropis kraepelini Leschke, 1914: 211, figs 5-7 (“Tjibodas”). Syn. nov.

Helix winteriana - Philippi 1843 in 1842-1845: 23, pl. II.1, fig. 7. - Pfeiffer 1851 in 1849-1853: pl. 95, figs 1-2; 1852 in 1849-1853: 162. — Martens 1867: 264 (in part).

Plectotropis sumatrana var. moussoniana - Leschke 1914: 211. (not Martens, 1867)

Landouria winteriana - van Benthem Jutting 1950: 463 (in part).

Landouria rotatoria - van Benthem Jutting 1950: 461 (in part). — Smith \& Djajasasmita 1988: 394, pl. 2 fig. 10. (not Pfeiffer, 1842)

Landouria cf. rotatoria - Köhler et al. in press: 6, figs 3A, 5 (in part). (not Pfeiffer, 1842)

\section{Diagnosis}

Landouria winteriana is characterized by a brownish-corneous, depressed conical, keeled shell with a wide umbilicus, a penis with a large globular bulge at the proximal end and a flagellum consisting of a crenated distal part and a short tapering proximal part.

\section{Material examined}

Type material

INDONESIA - West Java 1 syntype of Plectotropis kraepelini; Cibodas; $6^{\circ} 45^{\prime} \mathrm{S}, 1^{\circ} 7^{\circ} 01^{\prime} \mathrm{E}$; ZMH 98416 - neotype of Helix winteriana (designated here) (det. anat.) (voucher for L. rotatoria in van Benthem Jutting 1950); Padalarang, Gunung Masigit; $6^{\circ} 50^{\prime}$ S, $107^{\circ} 26^{\prime}$ E; 500 m a.s.l.; Jan. 1938; W.Ch. Voute leg.; measurements: $\mathrm{D}=12.6 \mathrm{~mm}, \mathrm{H}=6.9 \mathrm{~mm}$; ZMA 376193a.

\section{Other material}

INDONESIA - West Java - 1 spec. (det. anat.); Bogor, Cisarua, Halimun Salak National Park, $1166 \mathrm{~m}$ a.s.1.; $6^{\circ} 39^{\prime} 03^{\prime \prime} \mathrm{S}, 106^{\circ} 27^{\prime} 15^{\prime \prime} \mathrm{E}$; MZB 19235 • $1 \mathrm{spec}$. (voucher for van Benthem Jutting 1950); Bogor, Gunung Salak; 1200 m a.s.l.;ZMA $407749 \bullet 4$ spec.; Bogor, Cigudeg, Sipahang Cave (in Gudawang cave complex), agroforestry; $6^{\circ} 27^{\prime} 00^{\prime \prime} \mathrm{S}, 106^{\circ} 31^{\prime} 00^{\prime \prime} \mathrm{E}$; $91 \mathrm{~m}$ a.s.1.; MZB $17602 \cdot 7 \mathrm{spec}$.; Bogor, Ciampea, limestone hill Gunung Cibodas, secondary forest; 6033'05" S, 106 41'09" E; 296 m a.s.l.; MZB 19238 • 98 spec.; same data as for preceding; MZB $19239 \bullet 11$ spec.; same data as for preceding; MZB $19240 \bullet$ 6 spec.; same data as for preceding; ZMH 133425 • 30 spec.; same data as for preceding; ZMH 133426 - 4 spec.; same data as for preceding; ZMH $133427 \cdot 3$ spec.; Bogor, Ciampea, limestone hill Gunung Cibodas, inside Si Panjang Cave; 6³3'09" S, 106 41'13" E; 357 m a.s.1.; MZB 19279 • 3 spec.; Bogor, Ciampea, limestone hill Gunung Cibodas, Si Ase (Si Gajah) cave mouth; 6³3'08" S, $106^{\circ} 41^{\prime} 14^{\prime \prime}$ E; 
309 m a.s.l.; MZB 19249 - 1 spec. (voucher for P. sumatrana var. moussoniana in Leschke 1914); Ciampea; 6 $6^{\circ} 33^{\prime} \mathrm{S}, 106^{\circ} 42^{\prime} \mathrm{E}$; ZMH 20426 - 3 spec. (det. anat.); Bogor, Pabaton 25; 6 $35^{\prime} 22^{\prime \prime} \mathrm{S}$, $106^{\circ} 47^{\prime} 33^{\prime \prime}$ E; ZMA 369719 • 2 spec. (vouchers for P. sumatrana var. moussoniana in Leschke 1914); Bogor; 636' S, $106^{\circ} 48^{\prime}$ E; ZMH 20425 - 1 spec.; Bogor; 6³6' S, 106 $48^{\prime}$ E; $230 \mathrm{~m}$ a.s.1.; ZMA $407751 \bullet>10$ spec. (vouchers for van Benthem Jutting 1950); Bogor; $6^{\circ} 36^{\prime} \mathrm{S}, 106^{\circ} 48^{\prime} \mathrm{E}$; $250 \mathrm{~m}$ a.s.l.; ZMA 407628 - 1 spec. (voucher for van Benthem Jutting 1950); Bogor, botanical garden; $6^{\circ} 36^{\prime} \mathrm{S}$, $106^{\circ} 48^{\prime}$ E; 250 m a.s.l.; ZMA 407750 2 spec.; Sukabumi, Jampangtengah, Ciareuy, bamboo plantation; $7^{\circ} 01^{\prime} 49^{\prime \prime}$ S, $106^{\circ} 48^{\prime} 26^{\prime \prime}$ E; 573 m a.s.1.; MZB $19248 \bullet 1$ spec. (voucher for van Benthem Jutting 1950); Lake of Cigombong, forest near lake; $6^{\circ} 45^{\prime} \mathrm{S}, 106^{\circ} 49^{\prime} \mathrm{E}$; ZMA $407800 \cdot 2$ spec.; Sukabumi, Benda, Bodogol Nature Reserve at Gunung Pangrango, 800 m a.s.l.; 646'40" S, 10651'26" E; ZMH 140032 • 2 spec. (vouchers for van Benthem Jutting 1950); Bogor, Gunung Pancar, 800 m a.s.l.; 6 ${ }^{\circ} 35^{\prime}$ S, $106^{\circ} 54^{\prime}$ E; ZMA 407790 - 1 spec. (det. anat.); Bogor, saddle between Gunung Pancar and Gunung Paniisan; $6^{\circ} 35^{\prime}$ S, $106^{\circ} 54^{\prime}$ E; 500 m a.s.l.; ZMA 370986 • 5 spec.; Sukabumi, Nyalindung, near Bibijilan waterfall, secondary forest; 7001'27" S, $106^{\circ} 54^{\prime 2} 21^{\prime \prime}$ E; 696 m a s.l.; MZB $19247 \cdot 7$ spec.; Sukabumi, Nyalindung, Buniayu cave complex, Cipicung Cave, secondary forest; $7^{\circ} 02^{\prime} 10^{\prime \prime} \mathrm{S}, 106^{\circ} 54^{\prime} 21^{\prime \prime} \mathrm{E} ; 755 \mathrm{~m}$ a.s.l.; MZB 19244 • 7 spec.; Sukabumi, Nyalindung, Buniayu, secondary forest; 702'12" S, 10654'26" E; 795 m a.s.1.; MZB 19246 $3 \mathrm{spec}$.; same data as for preceding; ZMH $133422 \bullet 1 \mathrm{spec}$.; Sukabumi, Nyalindung, Buniayu, Altingia (Rasamala) Forest; $7^{\circ} 02^{\prime} 11^{\prime \prime} \mathrm{S}, 1^{\circ} 6^{\circ} 54^{\prime} 35^{\prime \prime} \mathrm{E} ; 805 \mathrm{~m}$ a.s.1.; MZB $19245 \cdot 1$ spec. (det. anat.); Bogor, Megamendung; 6 $6^{\circ} 8^{\prime} 35^{\prime \prime}$ S, 106 55'23" E; MZB $3618 \cdot 2$ spec. (vouchers for van Benthem Jutting 1950); Bogor, S of Cisarua; $6^{\circ} 41^{\prime} \mathrm{S}, 106^{\circ} 56^{\prime} \mathrm{E}$; $800 \mathrm{~m}$ a.s.1.; ZMA $407712 \cdot 2$ spec. (vouchers for van Benthem Jutting 1950); same data as for preceding; ZMA $407719 \bullet 1$ spec. (voucher for $P$. sumatrana var. moussoniana in Leschke 1914); Cibodas; $6^{\circ} 45^{\prime} \mathrm{S}, 107^{\circ} 01^{\prime} \mathrm{E}$; ZMH $20424 \bullet 1 \mathrm{spec}$.; Padalarang, Rajamandala; $6^{\circ} 50^{\prime}$ S, $107^{\circ} 21^{\prime}$ E; ZMA $413757 \bullet 1$ spec. (voucher for van Benthem Jutting 1950); Jampang, limestone rock near Sukanegara; $7^{\circ} 01^{\prime}$ S, $107^{\circ} 22^{\prime}$ E; 1000 m a.s.l.; ZMA $407746 \bullet$ 4 spec. (vouchers for van Benthem Jutting 1950); Garut; $7^{\circ} 01^{\prime}$ S, $107^{\circ} 25^{\prime}$ E; RMNH $309721 \bullet 6$ spec. (det. anat.); Padalarang, Gunung Masigit; $6^{\circ} 50^{\prime}$ S, $107^{\circ} 26^{\prime}$ E; 500 m a.s.1.; ZMA $376193 \cdot$ many spec. (vouchers for van Benthem Jutting 1950); Padalarang, Gunung Pawon; 649' S, $107^{\circ} 26^{\prime}$ E; 700 m a.s.1.; ZMA 407631 - 27 spec.; Padalarang, Gunung Pawon, shrubs and palm; 649'29" S, 107 26'12" E;

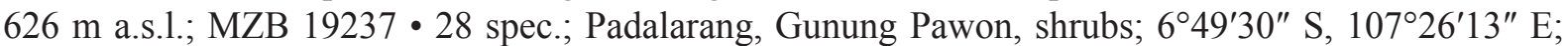
689 m a.s.l.; MZB 19243 • 15 spec.; same data as for preceding; ZMH 133422 • 1 spec.; Padalarang, Gunung Pawon, agroforestry; 649'31" S, 107²6'16" E; 697 m a.s.l.; MZB $19236 \bullet 11$ spec.; same data as for preceding; MZB 19254 • 4 spec.; Padalarang, bamboo; 6²9'36" S, 107²7'09" E; 784 m a.s.l.; MZB 19251 • 3 spec.; Padalarang, Gunung Hawu, agroforestry; 650'04" S, 107²7'31" E; 835 m a.s.1.; MZB 19291 • 36 spec.; Padalarang, Gunung Hawu, guava plantation; 6 $0^{\circ} 50^{\prime} 07^{\prime \prime} \mathrm{S}, 107^{\circ} 27^{\prime} 35^{\prime \prime} \mathrm{E}$; $787 \mathrm{~m}$ a.s.1.; MZB 19242 • 20 spec.; same data as for preceding; ZMH $133423 \bullet 15$ spec.; Padalarang, Gunung

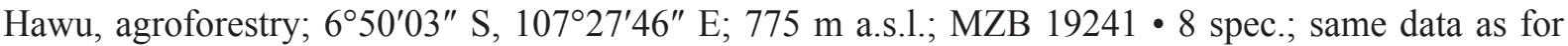
preceding; ZMH $133424 \cdot 4$ spec. (vouchers for van Benthem Jutting 1950); Cibuluh near Cisurupan, slope of Papandayan; $7^{\circ} 21^{\prime}$ S, $107^{\circ} 47^{\prime}$ E; RMNH $309739 \bullet 4$ spec.; Cibuluh near Cikajang, slope of Papandayan; $7^{\circ} 21^{\prime} \mathrm{S}, 107^{\circ} 48^{\prime} \mathrm{E}$; RMNH $309762 \cdot 7$ spec.; Tasikmalaya, Karangnunggal, surroundings of Kiara Jenggot Cave, agroforestry; $7^{\circ} 39^{\prime} 37^{\prime \prime}$ S, 108 $06^{\prime} 55^{\prime \prime}$ E; 234 m a.s.l.; MZB 19250 • 3 spec.; Tasikmalaya, Setiawaras, near Sambawa Cave, agroforestry; 7³5'02" S, 108 $10^{\circ} 58^{\prime \prime}$ E; $361 \mathrm{~m}$ a.s.l.; MZB 17789.

\section{Description}

Shell (Figs 2-3; Tables 3-4). Depressed conical, with 5-5.75 hardly convex whorls; protoconch almost smooth; teleoconch with fine, irregular wrinkles; apical side with indistinct incised spiral lines, umbilical side with more distinct spiral lines; scaly processes sparse, mainly at periphery; tuberculate around umbilicus; brownish-corneous; body whorl keeled; aperture rounded rhombic; upper insertion of peristome descending; peristome expanded, reflexed and slightly thickened; umbilicus slightly eccentric, wide, comprising $29-51 \%$ of shell diameter, hardly obscured by columellar edge. 


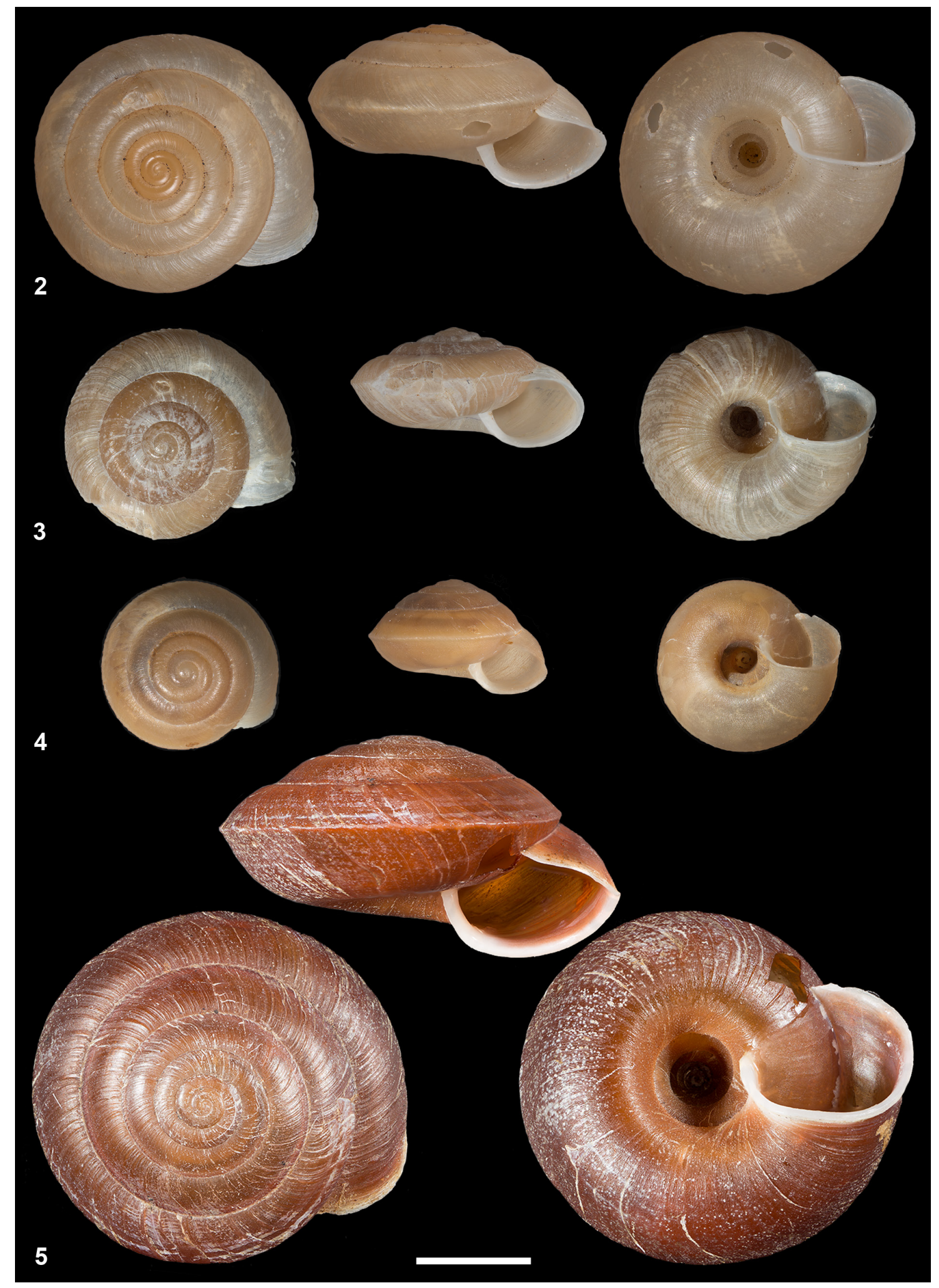

Figs 2-5. Shells of species of Landouria from Java. 2. L. winteriana (Pfeiffer, 1842), West Java, Padalarang, Gunung Masigit (neotype of Helix winteriana, ZMA 376193a). 3. L. winteriana, West Java, Cibodas (syntype of Plectotropis kraepelini Leschke, 1914, ZMH 98416). 4. L. conoidea (Leschke, 1914), West Java, Bogor (syntype, ZMH 98144). 5. L. rotatoria (Pfeiffer, 1842), West Java, Gunung Salak (neotype of Helix rotatoria, MZB 19205). Scale bar: $5 \mathrm{~mm}$. 
Genitalia (Figs 15, 29; Table 4). Atrium short; penis long, distal part cylindrical, proximal part globularly dilated; without penis coecum; membranaceous sheath around distal part of penis connected with proximal part of epiphallus by tissue; penial retractor runs from diaphragm to middle part of short cylindrical epiphallus; flagellum moderately long, distal part broader, apically with some indentions, apical part tapering towards its apical end; without node; vas deferens narrow and long; vagina long; oviduct short; peduncle of bursa copulatrix subdivided into a broader, rapidly tapering distal part and a narrower, longer proximal part, ending in an elongate oval bursa beside albumen gland. Right ommatophoral retractor runs between penis and vagina.

\section{Remarks}

Landouria winteriana and L. rotatoria are the oldest names for species of Landouria from the IndoAustralian Archipelago. Pfeiffer (1842) described both from Java without more exact locality data. Later, these names have been used for many different species from Java and other islands of the Indo-Australian Archipelago. Thus, it is important to clarify their identity. The original description of H. winteriana was based on material in Pfeiffer's own collection (Pfeiffer 1852 in 1849-1853). Pfeiffer's collection was acquired by H. Dohrn and his collection in the Stettin Museum was destroyed during the Second World War (Zilch 1967; Dance 1986). We could not locate type specimens of $H$. winteriana in other museums (e.g., the collection of von dem Busch in the Geosciences Collection of the University of Bremen, SMF, ZMH). Thus, a neotype has to be designated to clarify the taxonomic status and the type locality of $H$. winteriana.

We designate a specimen from Gunung Masigit near Padalarang in West Java (ZMA 376193a; Figs 2, 21), which agrees with the original description of Pfeiffer (1842) and the figures of Pfeiffer (1851 in 1849-1853: pl. 95, figs 1-2) in the depressed conical shell with an angular periphery and a wide umbilicus, as the neotype of Helix winteriana Pfeiffer, 1842. This specimen represents a species that is widespread in western Java, which was more intensively studied than other parts of Java in former times (and still today), so it is likely that the neotype actually belongs to the same species as the specimens described by Pfeiffer (1842).

Köhler et al. (in press) recently redescribed $L$. winteriana under the name $L$. cf. rotatoria (not Pfeiffer, 1842). The species from Timor named $L$. cf. winteriana by Köhler et al. (in press) is not closely related to $L$. winteriana. Although it is more closely related to species of Landouria from Java than to $L$. timorensis Köhler et al. in press or L. montana Köhler et al. in press from Timor (see Fig. 1; Köhler et al. in press: fig. 2), it is not identical with any of the species described here from Java, but probably represents a new species.

The only available syntype of P. kraepelini (Fig. 3) differs from usual L. winteriana in the smaller shell diameter at three whorls ( $4.0 \mathrm{~mm}$ vs $4.4-5.2 \mathrm{~mm}$ in other $L$. winteriana) and the slightly more pronounced keel. We suppose that $P$. kraepelini represents a small variety of $L$. winteriana. However, the specific identity of $P$. kraepelini and $L$. winteriana should be checked by an anatomical examination of specimens resembling the syntype of $P$. kraepelini from Cibodas, its type locality.

Landouria winteriana differs from L. sukoliloensis sp. nov. in the angular body whorl, the not convoluted distal part of the penis, the short epiphallus and the flagellum with a broader distal part with external indentions. This species occurs sympatrically with $L$. petrukensis sp. nov., from which it differs in the larger size and the angular, less sharply keeled periphery.

\section{Distribution}

Anatomically determined samples of $L$. winteriana are known from Bogor Regency and Padalarang in the mountain region in West Java (Fig. 52). The shells from Tasikmalaya Regency resemble the 
Table 3 (continued on next 3 pages). Shell measurements for species of Landouria Godwin-Austen, 1918 (in mm). Abbreviations: $\mathrm{D}=$ shell diameter; D3W = diameter of the third whorl; da = diameter of the aperture; $\mathrm{H}=$ shell height; ha = height of the aperture; $\max =$ maximum; $\min =$ minimum; $n=$ number of measured spec.; std = standard deviation; $\mathrm{U}=$ diameter of the umbilicus; $\mathrm{W}=$ number of whorls.

\begin{tabular}{|c|c|c|c|c|c|c|c|c|c|c|c|c|}
\hline & D & D3W & $\mathbf{H}$ & da & ha & $\mathbf{U}$ & $\mathbf{W}$ & $\mathbf{D} / \mathbf{H}$ & $\mathbf{U} / \mathbf{D}$ & D/da & H/ha & $\mathrm{da} / \mathrm{ha}$ \\
\hline \multicolumn{13}{|c|}{ Landouria winteriana; several localities; $n=20$} \\
\hline $\min$ & 9.7 & 4.4 & 5.5 & 3.7 & 4.0 & 3.4 & 5 & 1.55 & 0.29 & 2.26 & 1.24 & 0.94 \\
\hline $\max$ & 12.6 & 5.2 & 7.8 & 5.5 & 5.2 & 6.0 & 5.75 & 2.07 & 0.51 & 2.60 & 1.55 & 1.15 \\
\hline mean & 11.8 & 4.8 & 6.7 & 5.0 & 4.8 & 4.1 & 5.3 & 1.76 & 0.35 & 2.39 & 1.41 & 1.04 \\
\hline std & 0.7 & 0.2 & 0.6 & 0.4 & 0.3 & 0.7 & 0.2 & 0.11 & 0.06 & 0.08 & 0.08 & 0.05 \\
\hline \multicolumn{13}{|c|}{ Landouria conoidea ; Bogor; $n=3$} \\
\hline $\min$ & 7.6 & 3.6 & 5.2 & 3.4 & 3.2 & 2.5 & 4.75 & 1.48 & 0.30 & 2.16 & 1.55 & 1.04 \\
\hline $\max$ & 8.7 & 4.4 & 5.6 & 3.8 & 3.7 & 2.6 & 5 & 1.54 & 0.33 & 2.27 & 1.59 & 1.12 \\
\hline mean & 8.1 & 4.1 & 5.3 & 3.6 & 3.4 & 2.5 & 4.9 & 1.52 & 0.31 & 2.23 & 1.57 & 1.07 \\
\hline std & 0.5 & 0.4 & 0.3 & 0.2 & 0.2 & 0.1 & 0.1 & 0.03 & 0.01 & 0.06 & 0.02 & 0.04 \\
\hline \multicolumn{13}{|c|}{ Landouria rotatoria; Gunung Gede; Gunung Salak; $n=22$} \\
\hline $\min$ & 15.6 & 4.5 & 8.3 & 6.8 & 6.5 & 4.1 & 5.75 & 1.57 & 0.24 & 2.19 & 1.17 & 0.96 \\
\hline $\max$ & 19.1 & 5.8 & 10.6 & 8.5 & 8.0 & 5.8 & 6.5 & 2.09 & 0.32 & 2.38 & 1.47 & 1.12 \\
\hline mean & 17.4 & 5.1 & 9.5 & 7.6 & 7.2 & 5.0 & 6.1 & 1.85 & 0.29 & 2.28 & 1.31 & 1.06 \\
\hline std & 0.8 & 0.3 & 0.6 & 0.4 & 0.4 & 0.5 & 0.2 & 0.12 & 0.02 & 0.05 & 0.08 & 0.04 \\
\hline \multicolumn{13}{|c|}{ Landouria naggsi sp. nov.; Sukabumi; $n=4$} \\
\hline $\min$ & 11.6 & 4.2 & 8.5 & 5.0 & 4.9 & 3.3 & 6.25 & 1.21 & 0.28 & 2.07 & 1.55 & 0.92 \\
\hline $\max$ & 14.0 & 5.0 & 10.6 & 5.8 & 6.1 & 4.7 & 7 & 1.37 & 0.33 & 2.40 & 1.99 & 1.16 \\
\hline mean & 12.5 & 4.5 & 9.6 & 5.5 & 5.5 & 3.9 & 6.6 & 1.31 & 0.31 & 2.28 & 1.74 & 1.00 \\
\hline std & 1.1 & 0.4 & 0.9 & 0.4 & 0.5 & 0.6 & 0.3 & 0.07 & 0.02 & 0.14 & 0.19 & 0.11 \\
\hline \multicolumn{13}{|c|}{ Landouria epiplatia; Jampang; near Palabuhan Ratu Bay; $n=8$} \\
\hline $\min$ & 11.8 & 4.5 & 5.7 & 4.4 & 4.1 & 3.8 & 5.25 & 2.03 & 0.30 & 2.61 & 1.02 & 1.03 \\
\hline $\max$ & 17.0 & 5.7 & 6.9 & 6.2 & 6.0 & 7.3 & 6.25 & 2.94 & 0.43 & 2.74 & 1.41 & 1.19 \\
\hline mean & 15.3 & 4.9 & 6.0 & 5.7 & 5.2 & 5.7 & 5.7 & 2.52 & 0.37 & 2.66 & 1.18 & 1.11 \\
\hline std & 2.0 & 0.4 & 0.4 & 0.7 & 0.7 & 1.5 & 0.4 & 0.34 & 0.06 & 0.05 & 0.14 & 0.05 \\
\hline \multicolumn{13}{|c|}{ Landouria parahyangensis sp. nov.; Karangnunggal; $n=7$} \\
\hline $\min$ & 14.7 & 4.3 & 8.4 & 5.3 & 5.5 & 5.8 & 6.5 & 1.69 & 0.39 & 2.55 & 1.42 & 0.95 \\
\hline $\max$ & 16.9 & 5.0 & 9.3 & 6.3 & 6.2 & 7.4 & 7 & 1.94 & 0.45 & 2.82 & 1.60 & 1.10 \\
\hline mean & 15.7 & 4.5 & 8.7 & 5.8 & 5.8 & 6.5 & 6.8 & 1.80 & 0.42 & 2.71 & 1.50 & 1.00 \\
\hline std & 0.9 & 0.3 & 0.4 & 0.4 & 0.3 & 0.6 & 0.2 & 0.09 & 0.02 & 0.09 & 0.05 & 0.05 \\
\hline \multicolumn{13}{|c|}{ Landouria nusakambangensis sp. nov.; Nusa Kambangan Island; $n=8$} \\
\hline $\min$ & 11.9 & 4.4 & 6.6 & 4.8 & 4.6 & 3.9 & 5.25 & 1.65 & 0.32 & 2.36 & 1.29 & 0.95 \\
\hline $\max$ & 15.0 & 5.4 & 9.1 & 6.4 & 6.2 & 5.8 & 6 & 1.83 & 0.40 & 2.52 & 1.56 & 1.09 \\
\hline mean & 13.5 & 4.9 & 7.9 & 5.6 & 5.4 & 4.9 & 5.7 & 1.72 & 0.36 & 2.43 & 1.46 & 1.03 \\
\hline std & 1.3 & 0.3 & 0.8 & 0.6 & 0.6 & 0.8 & 0.3 & 0.07 & 0.03 & 0.05 & 0.09 & 0.05 \\
\hline
\end{tabular}


Table 3 (continued). Shell measurements for species of Landouria Godwin-Austen, 1918.

\begin{tabular}{|c|c|c|c|c|c|c|c|c|c|c|c|c|}
\hline & $\mathbf{D}$ & D3W & $\mathbf{H}$ & da & ha & $\mathbf{U}$ & $\mathbf{W}$ & $\mathbf{D} / \mathbf{H}$ & $\mathbf{U} / \mathbf{D}$ & $\mathrm{D} / \mathrm{da}$ & H/ha & da/ha \\
\hline \multicolumn{13}{|c|}{ Landouria petrukensis sp. nov.; Karangbolong; $n=13$} \\
\hline $\min$ & 8.1 & 3.7 & 5.2 & 3.6 & 3.4 & 2.2 & 4.75 & 1.49 & 0.24 & 2.05 & 1.35 & 1.02 \\
\hline $\max$ & 9.8 & 4.3 & 6.3 & 4.6 & 4.1 & 3.3 & 5.5 & 1.79 & 0.37 & 2.44 & 1.63 & 1.18 \\
\hline mean & 9.0 & 3.9 & 5.5 & 4.0 & 3.7 & 2.5 & 5.1 & 1.62 & 0.27 & 2.22 & 1.48 & 1.08 \\
\hline std & 0.6 & 0.2 & 0.4 & 0.3 & 0.3 & 0.3 & 0.2 & 0.08 & 0.03 & 0.09 & 0.09 & 0.05 \\
\hline \multicolumn{13}{|c|}{ Landouria tholiformis sp. nov.; Gunung Kidul; $n=7$} \\
\hline $\min$ & 13.0 & 4.2 & 7.4 & 5.1 & 4.9 & 4.5 & 6 & 1.77 & 0.32 & 2.40 & 1.37 & 1.00 \\
\hline $\max$ & 14.4 & 4.5 & 7.8 & 5.8 & 5.7 & 5.6 & 6.25 & 1.88 & 0.40 & 2.57 & 1.52 & 1.06 \\
\hline mean & 13.9 & 4.4 & 7.7 & 5.6 & 5.4 & 4.9 & 6.1 & 1.82 & 0.35 & 2.48 & 1.42 & 1.04 \\
\hline std & 0.5 & 0.1 & 0.2 & 0.2 & 0.3 & 0.4 & 0.1 & 0.04 & 0.03 & 0.05 & 0.05 & 0.02 \\
\hline \multicolumn{13}{|c|}{ Landouria abdidalem sp. nov.; Kulonprogo; $n=11$} \\
\hline $\min$ & 13.1 & 4.3 & 6.3 & 5.1 & 4.6 & 4.6 & 5.75 & 1.93 & 0.33 & 2.42 & 1.29 & 1.01 \\
\hline $\max$ & 16.4 & 4.8 & 7.8 & 6.1 & 5.8 & 7.0 & 6 & 2.14 & 0.48 & 2.79 & 1.43 & 1.13 \\
\hline mean & 14.0 & 4.5 & 6.9 & 5.5 & 5.1 & 5.4 & 5.9 & 2.03 & 0.38 & 2.56 & 1.35 & 1.07 \\
\hline std & 0.9 & 0.2 & 0.5 & 0.3 & 0.4 & 0.8 & 0.1 & 0.07 & 0.04 & 0.10 & 0.04 & 0.04 \\
\hline \multicolumn{13}{|c|}{ Landouria madurensis sp. nov.; Madura Island; $n=3$} \\
\hline $\min$ & 11.4 & 3.9 & 5.5 & 4.5 & 4.5 & 4.3 & 5.5 & 1.75 & 0.38 & 2.45 & 1.23 & 1.00 \\
\hline $\max$ & 12.0 & 4.5 & 6.5 & 4.7 & 4.6 & 4.5 & 5.75 & 2.11 & 0.40 & 2.57 & 1.46 & 1.04 \\
\hline mean & 11.6 & 4.1 & 5.9 & 4.6 & 4.5 & 4.4 & 5.7 & 1.98 & 0.39 & 2.52 & 1.31 & 1.02 \\
\hline std & 0.3 & 0.3 & 0.5 & 0.1 & 0.1 & 0.1 & 0.1 & 0.20 & 0.01 & 0.06 & 0.13 & 0.02 \\
\hline \multicolumn{13}{|c|}{ Landouria sewuensis sp. nov.; several hills in Gunung Kidul; $n=8$} \\
\hline $\min$ & 11.4 & 3.9 & 6.3 & 5.0 & 4.4 & 3.5 & 5.25 & 1.61 & 0.30 & 2.21 & 1.25 & 1.06 \\
\hline $\max$ & 16.3 & 5.3 & 8.7 & 7.2 & 6.3 & 6.0 & 6 & 2.25 & 0.40 & 2.57 & 1.50 & 1.23 \\
\hline mean & 13.7 & 4.6 & 7.2 & 5.9 & 5.2 & 4.9 & 5.7 & 1.90 & 0.36 & 2.35 & 1.39 & 1.12 \\
\hline std & 1.8 & 0.5 & 0.7 & 0.8 & 0.6 & 0.8 & 0.3 & 0.20 & 0.03 & 0.12 & 0.09 & 0.05 \\
\hline \multicolumn{13}{|c|}{ Landouria sukoliloensis sp. nov.; Pati; Grobogan; $n=20$} \\
\hline $\min$ & 10.4 & 3.6 & 6.0 & 4.7 & 3.9 & 2.5 & 5 & 1.60 & 0.22 & 2.04 & 1.26 & 1.04 \\
\hline $\max$ & 12.3 & 4.6 & 7.4 & 5.6 & 4.8 & 4.8 & 5.75 & 1.93 & 0.41 & 2.46 & 1.67 & 1.20 \\
\hline mean & 11.2 & 4.0 & 6.5 & 5.1 & 4.5 & 3.3 & 5.5 & 1.72 & 0.29 & 2.22 & 1.47 & 1.14 \\
\hline std & 0.5 & 0.2 & 0.4 & 0.3 & 0.3 & 0.8 & 0.2 & 0.09 & 0.07 & 0.09 & 0.09 & 0.05 \\
\hline \multicolumn{13}{|c|}{ Landouria tonywhitteni sp. nov.; Grobogan; $n=10$} \\
\hline $\min$ & 14.3 & 4.8 & 7.0 & 6.0 & 5.5 & 5.5 & 5.75 & 1.93 & 0.32 & 2.30 & 1.11 & 1.07 \\
\hline $\max$ & 17.6 & 5.4 & 8.5 & 7.4 & 6.7 & 6.7 & 6 & 2.34 & 0.41 & 2.61 & 1.46 & 1.20 \\
\hline mean & 16.1 & 4.9 & 7.5 & 6.6 & 6.0 & 5.9 & 5.8 & 2.14 & 0.37 & 2.43 & 1.27 & 1.11 \\
\hline std & 1.0 & 0.2 & 0.4 & 0.4 & 0.4 & 0.4 & 0.1 & 0.16 & 0.03 & 0.08 & 0.10 & 0.04 \\
\hline \multicolumn{13}{|c|}{ Landouria nodifera sp. nov.; Pati; $n=13$} \\
\hline $\min$ & 14.7 & 5.0 & 6.4 & 5.8 & 4.6 & 3.9 & 5 & 1.93 & 0.26 & 2.33 & 1.14 & 1.00 \\
\hline $\max$ & 16.1 & 5.5 & 8.1 & 6.9 & 6.3 & 5.6 & 5.75 & 2.29 & 0.38 & 2.54 & 1.39 & 1.36 \\
\hline mean & 15.2 & 5.3 & 7.2 & 6.3 & 5.8 & 4.8 & 5.5 & 2.12 & 0.32 & 2.42 & 1.24 & 1.09 \\
\hline std & 0.4 & 0.1 & 0.5 & 0.3 & 0.4 & 0.4 & 0.2 & 0.10 & 0.03 & 0.07 & 0.07 & 0.09 \\
\hline
\end{tabular}


Table 3 (continued). Shell measurements for species of Landouria Godwin-Austen, 1918.

\begin{tabular}{|c|c|c|c|c|c|c|c|c|c|c|c|c|}
\hline & D & D3W & $\mathbf{H}$ & da & ha & $\mathbf{U}$ & $\mathbf{W}$ & $\mathbf{D} / \mathbf{H}$ & $\mathbf{U} / \mathbf{D}$ & $\mathrm{D} / \mathrm{da}$ & H/ha & da/ha \\
\hline \multicolumn{13}{|c|}{ Landouria pacitanensis sp. nov.; Pacitan; $n=16$} \\
\hline $\min$ & 10.6 & 3.4 & 6.2 & 4.1 & 4.1 & 3.0 & 5.25 & 1.57 & 0.28 & 2.16 & 1.19 & 0.93 \\
\hline $\max$ & 14.7 & 4.8 & 8.2 & 6.2 & 5.8 & 4.8 & 6 & 2.18 & 0.34 & 2.65 & 1.56 & 1.17 \\
\hline mean & 13.1 & 4.4 & 7.1 & 5.4 & 5.2 & 4.0 & 5.7 & 1.84 & 0.31 & 2.39 & 1.36 & 1.04 \\
\hline std & 1.4 & 0.4 & 0.6 & 0.6 & 0.5 & 0.6 & 0.3 & 0.18 & 0.02 & 0.12 & 0.09 & 0.06 \\
\hline \multicolumn{13}{|c|}{ Landouria intumescens; Tuban; Bojonegoro; Surabaja; $n=20$} \\
\hline $\min$ & 11.6 & 4.2 & 6.1 & 5.5 & 4.7 & 2.8 & 5 & 1.50 & 0.22 & 2.10 & 1.22 & 0.98 \\
\hline $\max$ & 14.1 & 5.3 & 8.7 & 6.4 & 6.2 & 4.5 & 5.75 & 1.98 & 0.34 & 2.33 & 1.60 & 1.19 \\
\hline mean & 13.2 & 4.7 & 7.5 & 5.9 & 5.5 & 3.6 & 5.3 & 1.76 & 0.27 & 2.21 & 1.38 & 1.09 \\
\hline std & 0.7 & 0.3 & 0.6 & 0.2 & 0.4 & 0.4 & 0.2 & 0.13 & 0.03 & 0.06 & 0.10 & 0.05 \\
\hline \multicolumn{13}{|c|}{ Landouria moussoniana; Wonosari; $n=6$} \\
\hline $\min$ & 10.2 & 4.0 & 5.5 & 4.2 & 4.1 & 2.7 & 5 & 1.73 & 0.37 & 2.60 & 1.52 & 0.99 \\
\hline $\max$ & 12.0 & 4.4 & 6.7 & 4.6 & 4.6 & 4.6 & 6.25 & 1.81 & 0.38 & 2.74 & 1.57 & 1.05 \\
\hline mean & 11.1 & 4.2 & 6.3 & 4.5 & 4.4 & 3.7 & 5.7 & 1.77 & 0.38 & 2.67 & 1.54 & 1.02 \\
\hline std & 0.3 & 0.3 & 0.1 & 0.3 & 0.1 & 0.0 & 0.2 & 0.06 & 0.01 & 0.10 & 0.04 & 0.05 \\
\hline \multicolumn{13}{|c|}{ Landouria schepmani; Sukapura; Gunung Bungbulang; $n=2$} \\
\hline lectotype & 10.9 & 4.3 & 6.2 & 4.6 & 4.2 & 2.3 & 5.25 & 1.76 & 0.21 & 2.36 & 1.48 & 1.11 \\
\hline paralectotype & 10.5 & 4.3 & 6.1 & 4.7 & 4.1 & 2.2 & 5 & 1.72 & 0.21 & 2.23 & 1.49 & 1.15 \\
\hline \multicolumn{13}{|c|}{ Landouria monticola; Gunung Cikuray; Java; $n=5$} \\
\hline $\min$ & 8.5 & 3.7 & 5.3 & 3.7 & 3.0 & 1.6 & 4.5 & 1.49 & 0.18 & 2.12 & 1.49 & 1.05 \\
\hline $\max$ & 9.5 & 5.0 & 6.0 & 4.1 & 3.9 & 2.1 & 5.25 & 1.60 & 0.25 & 2.32 & 2.00 & 1.37 \\
\hline mean & 9.0 & 4.4 & 5.8 & 4.0 & 3.4 & 1.8 & 4.8 & 1.56 & 0.21 & 2.25 & 1.73 & 1.21 \\
\hline std & 0.4 & 0.5 & 0.3 & 0.2 & 0.4 & 0.2 & 0.4 & 0.04 & 0.03 & 0.08 & 0.23 & 0.14 \\
\hline \multicolumn{13}{|c|}{ Landouria smimensis; several localities; $n=14$} \\
\hline $\min$ & 6.3 & 3.6 & 4.1 & 3.1 & 3.1 & 1.3 & 4.5 & 1.28 & 0.18 & 1.78 & 1.21 & 0.92 \\
\hline $\max$ & 11.9 & 4.4 & 8.4 & 5.3 & 5.1 & 3.3 & 5.5 & 1.64 & 0.32 & 2.63 & 1.65 & 1.08 \\
\hline mean & 8.2 & 3.8 & 5.6 & 3.8 & 3.7 & 2.0 & 4.9 & 1.48 & 0.25 & 2.17 & 1.49 & 1.01 \\
\hline std & 1.8 & 0.2 & 1.2 & 0.7 & 0.7 & 0.7 & 0.4 & 0.09 & 0.04 & 0.19 & 0.13 & 0.05 \\
\hline \multicolumn{13}{|c|}{ Landouria leucochila } \\
\hline holotype & 16.3 & 5.8 & 8.5 & 6.9 & 4.8 & 4.9 & 5.25 & 1.92 & 0.30 & 2.36 & 1.79 & 1.45 \\
\hline \multicolumn{13}{|c|}{ Landouria zonifera sp. nov.; Kulonprogo; $n=2$} \\
\hline $\min$ & 12.5 & 3.9 & 6.9 & 4.8 & 5.4 & 5.0 & 5.5 & 1.73 & 0.40 & 2.32 & 1.28 & 0.89 \\
\hline $\max$ & 13.2 & 4.8 & 7.6 & 5.7 & 5.6 & 5.3 & 5.75 & 1.81 & 0.40 & 2.61 & 1.37 & 1.02 \\
\hline mean & 12.8 & 4.3 & 7.2 & 5.2 & 5.5 & 5.1 & 5.6 & 1.77 & 0.40 & 2.47 & 1.32 & 0.95 \\
\hline std & 0.5 & 0.6 & 0.5 & 0.6 & 0.1 & 0.2 & 0.2 & 0.06 & 0.00 & 0.20 & 0.06 & 0.09 \\
\hline \multicolumn{13}{|c|}{ Landouria ciliocincta; several localities; $n=9$} \\
\hline $\min$ & 12.4 & 3.8 & 6.0 & 5.7 & 5.6 & 3.1 & 5 & 1.86 & 0.22 & 2.08 & 1.07 & 1.03 \\
\hline $\max$ & 15.6 & 4.5 & 8.3 & 7.1 & 6.5 & 4.1 & 6 & 2.12 & 0.30 & 2.27 & 1.29 & 1.10 \\
\hline mean & 13.6 & 4.3 & 7.0 & 6.2 & 5.8 & 3.7 & 5.4 & 1.94 & 0.27 & 2.18 & 1.21 & 1.07 \\
\hline std & 1.0 & 0.2 & 0.7 & 0.4 & 0.3 & 0.3 & 0.3 & 0.09 & 0.03 & 0.06 & 0.07 & 0.03 \\
\hline
\end{tabular}


Table 3 (continued). Shell measurements for species of Landouria Godwin-Austen, 1918.

\begin{tabular}{|c|c|c|c|c|c|c|c|c|c|c|c|c|}
\hline & D & D3W & $\mathbf{H}$ & da & ha & $\mathbf{U}$ & W & $\mathrm{D} / \mathrm{H}$ & U/D & $\mathrm{D} / \mathrm{da}$ & H/ha & da/ha \\
\hline \multicolumn{13}{|c|}{ Landouria pakidulan sp. nov.; Pangandaran; Nusa Kambangan Island; $n=4$} \\
\hline $\min$ & 12.5 & 4.6 & 6.9 & 5.7 & 5.4 & 3.0 & 5.25 & 1.70 & 0.24 & 2.05 & 1.20 & 1.05 \\
\hline $\max$ & 14.0 & 4.7 & 8.1 & 6.8 & 6.1 & 4.1 & 5.5 & 1.86 & 0.30 & 2.18 & 1.43 & 1.17 \\
\hline mean & 13.4 & 4.6 & 7.4 & 6.3 & 5.7 & 3.6 & 5.4 & 1.81 & 0.27 & 2.13 & 1.30 & 1.10 \\
\hline std & 0.7 & 0.0 & 0.5 & 0.5 & 0.3 & 0.4 & 0.1 & 0.07 & 0.02 & 0.07 & 0.10 & 0.05 \\
\hline \multicolumn{13}{|c|}{ Landouria dharmai sp. nov.; Tulungagung, Popoh } \\
\hline holotype & 9.9 & 3.9 & 7.2 & 5.3 & 4.5 & 1.8 & 5.5 & 1.39 & 0.18 & 1.88 & 1.57 & 1.17 \\
\hline \multicolumn{13}{|c|}{ Landouria davini; Baturraden, Pancuran Tujuh } \\
\hline holotype & 12.4 & 5.7 & 7.1 & 5.0 & 4.8 & 3.6 & 5 & 1.73 & 0.29 & 2.48 & 1.48 & 1.04 \\
\hline \multicolumn{13}{|c|}{ Landouria menorehensis sp. nov.; Kulonprogo, Sibolong; $n=9$} \\
\hline $\min$ & 12.4 & 4.3 & 8.3 & 5.9 & 5.5 & 2.2 & 5.25 & 1.31 & 0.16 & 1.85 & 1.33 & 0.98 \\
\hline $\max$ & 14.5 & 5.2 & 10.4 & 7.5 & 7.3 & 3.0 & 5.75 & 1.51 & 0.24 & 2.08 & 1.61 & 1.19 \\
\hline mean & 13.4 & 4.6 & 9.3 & 6.8 & 6.3 & 2.5 & 5.5 & 1.44 & 0.19 & 1.96 & 1.48 & 1.09 \\
\hline std & 0.7 & 0.3 & 0.7 & 0.6 & 0.6 & 0.2 & 0.1 & 0.06 & 0.02 & 0.08 & 0.10 & 0.06 \\
\hline
\end{tabular}

anatomically determined samples from Bogor Regency and Padalarang. However, their specific identity has to be checked anatomically. In addition, we have anatomically determined a sample from Rakata Island (3 spec. (vouchers for Smith \& Djajasasmita 1988), Krakatau Islands, 60' $08^{\prime} 46^{\prime \prime} \mathrm{S}, 105^{\circ} 26^{\prime} 23^{\prime \prime} \mathrm{E}$, MZB 9354).

Landouria winteriana has also been recorded from Thailand to the Philippines and the Maluku Islands (Martens 1867; Rensch 1932; van Benthem Jutting 1941, 1950; Vermeulen \& Whitten 1998; Boonngam et al. 2008). Besides on Java, L. winteriana might occur on Sumatra, but records from other islands refer to other species.

Landouria conoidea (Leschke, 1914) comb. nov.

Figs 4, 6, 53; Tables 3-4

Plectotropis conoidea Leschke, 1914: 212, figs 8-9 (“Buitenzorg”).

Landouria rotatoria - van Benthem Jutting 1950: 461 (in part). (not Pfeiffer, 1842)

\section{Diagnosis}

Landouria conoidea is characterized by a small, conical, sharply keeled shell with tubercles all over the shell surface.

\section{Material examined}

Syntypes

INDONESIA • 8 spec.; West Java, Bogor; 6³6' S, 106²48' E; ZMH 98144.

\section{Other material}

INDONESIA • 10 spec.; West Java, Bogor; 6³6' S, 106²48' E; ZMH 98145. 


\section{Description}

SHELl (Figs 4, 6; Tables 3-4). Conical, with 4.75-5 slightly convex whorls; protoconch almost smooth; teleoconch with fine, irregular wrinkles; apical side without distinct incised spiral lines, umbilical side with indistinct spiral lines; with tubercles all over shell surface that carry scaly processes or hairs mainly at periphery; tuberculate around umbilicus; brownish-corneous; body whorl sharply keeled at beginning;

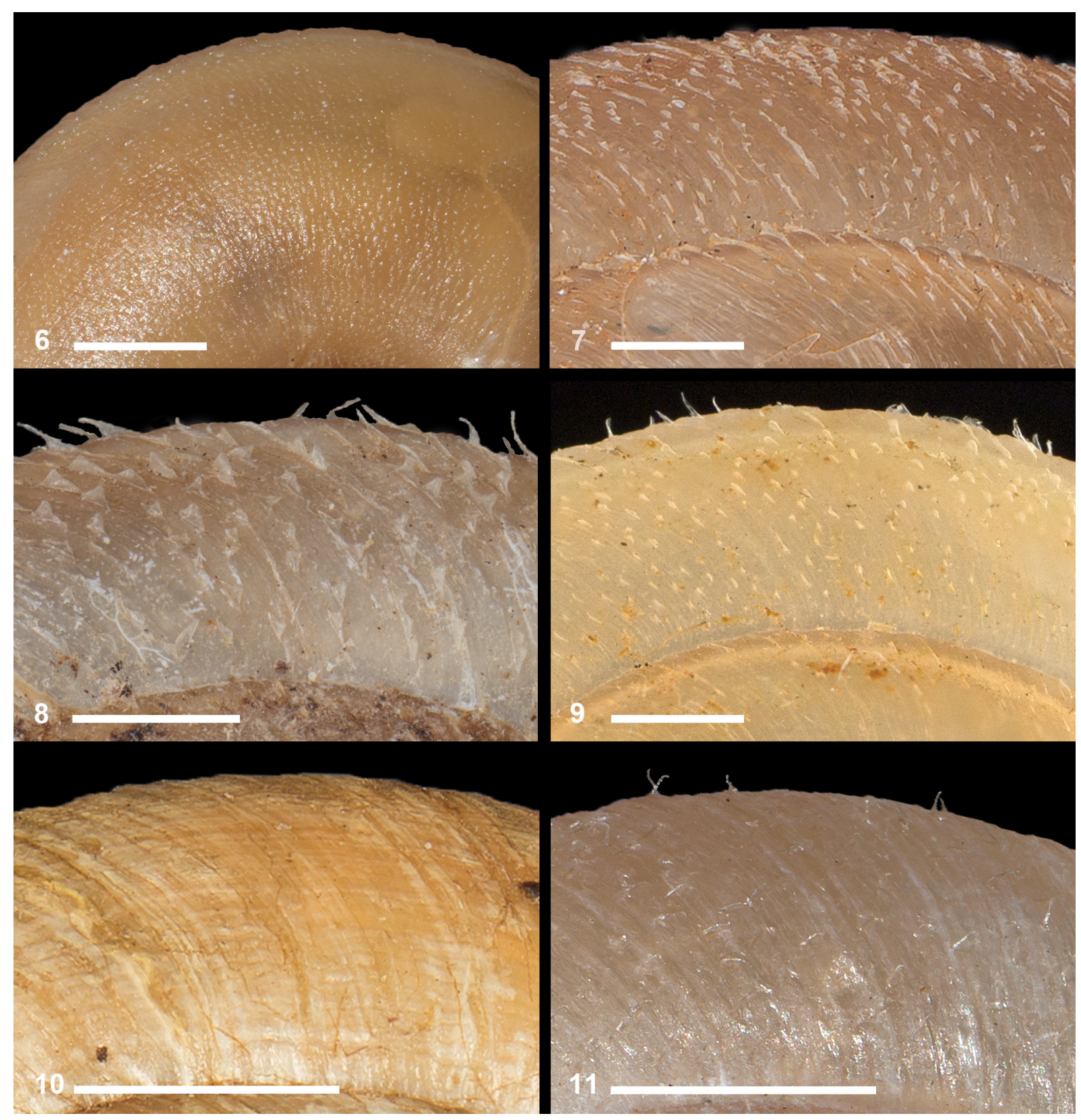

Figs 6-11. Shell sculpture of species of Landouria from Java (umbilical side of shell in 6; apical side in 7-11). 6. L. conoidea (Leschke, 1914), West Java, Bogor (syntype, ZMH 98144). 7. L. tholiformis sp. nov., Yogyakarta, Gunung Kidul, Tepus, near Poktunggal Beach (holotype, MZB 17999). 8. L. madurensis sp. nov., East Java, Madura Island, Sampang, Nepa Monkey lowland rainforest (paratype, MZB 19209). 9. L. pacitanensis sp. nov., East Java, Pacitan, Srau Beach (paratype, ZMH 148510). 10. L. monticola van Benthem Jutting, 1950, West Java, Gunung Cikuray (paratype, SMF 8880). 11. L. smimensis (Mousson, 1848), East Java, Tosari (ZMA 390881). Scale bars: $1 \mathrm{~mm}$. 
aperture almost circular; upper insertion of peristome slightly descending; peristome expanded, reflexed and slightly thickened; umbilicus hardly eccentric, wide, comprising $30-43 \%$ of shell diameter, hardly obscured by columellar edge.

Genitalia. Unknown.

\section{Remarks}

Landouria conoidea differs from $L$. winteriana, which occurs in the same region in West Java, in the smaller shell diameter (7.6-8.7 $\mathrm{mm}$ vs $9.7-12.6 \mathrm{~mm}$ in $L$. winteriana), the higher shell form $(\mathrm{D} / \mathrm{H}$ $1.48-1.54$ vs $1.55-2.07$ in $L$. winteriana), the more pronounced keel and the presence of tubercles all over the shell surface (Fig. 6).

With regard to the conical shell form, L. conoidea resembles L. naggsi sp. nov. (Fig. 25), from which it differs in the less elevated (D/H 1.48-1.54 vs 1.21-1.37 in L. naggsi sp. nov.), smaller shell (D 7.6$8.7 \mathrm{~mm}$ vs $11.6-14.0 \mathrm{~mm}$ in $L$. naggsi sp. nov.) with fewer whorls (4.75-5 vs $6.25-7$ in $L$. naggsi sp. nov.), the more pronounced keel and the shell sculpture consisting of tubercles vs longish scales in L. naggsi sp. nov.

\section{Distribution}

Landouria conoidea is so far known only from Bogor in West Java (Fig. 53).

Landouria rotatoria (Pfeiffer, 1842)

Figs 5, 12-14, 16, 30, 54; Tables 3-4

Helix rotatoria Pfeiffer 1842: 38 ("Java").

Plectotropis leucomphala Möllendorff, 1897: 66 (“Java”; more exactly (Zilch 1966): “W-Java: Gunung Gedeh (4000')").

Plectotropis leucomphala f. subfasciata Möllendorff, 1897: 67 (“Java"; more exactly (Zilch 1966): "W-Java: Gunung Gedeh (3000') ").

Plectotropis leucomphala f. castanea Möllendorff, 1897: 67 ("Java"; more exactly (Zilch 1966): "W-Java: Gunung Salak").

Helix rotatoria - von dem Busch in Philippi 1842 in 1842-1845: 10, pl. I.4, fig. 5. - Pfeiffer 1851 in 1849-1853: pl. 95, figs 3-5; 1852 in 1849-1853: 163.

Helix (Plectotropis) rotatoria - Boettger 1890: 144. — Martens 1892: 234. — Wiegmann 1893: 166, pl. 12, figs 11-14.

Landouria rotatoria - van Benthem Jutting 1950: 461 (in part). — Zilch 1966: 294, pl. 7, figs 3-5 (in part).

\section{Diagnosis}

Landouria rotatoria is characterized by a large, depressed conical, keeled shell, a penis with a small globular bulge at the proximal end and an externally smooth, longish lemon-shaped flagellum.

\section{Material examined}

\section{Type material}

INDONESIA - West Java - neotype of Helix rotatoria (designated here) (det. anat.); Bogor, Blok kancil, Gunung Salak, Halimun Salak National Park; 644'42" S, 106²' $42^{\prime \prime}$ E; 1166 m a.s.1.; 17 Jun. 2015; A.S. Nurinsiyah, I. Fauziah, T. Setiadi and Y. Prasetio leg.; measurements: D $=17.0 \mathrm{~mm}$, $\mathrm{H}=9.5 \mathrm{~mm}$; MZB $19205 \cdot$ holotype of Plectotropis leucomphala f. castanea; Gunung Salak; $6^{\circ} 44^{\prime}$ S, 
$106^{\circ} 42^{\prime}$ E; SMF 8916 - holotype of Plectotropis leucomphala f. subfasciata; Gunung Gede; $6^{\circ} 47^{\prime}$ S, $106^{\circ} 59^{\prime}$ E; ca 900 m a.s.1.; SMF 8914 • holotype of Plectotropis leucomphala; Gunung Gede; $6^{\circ} 47^{\prime}$ S, $106^{\circ} 59^{\prime}$ E; ca 1200 m a.s.1.; SMF 8912.

\section{Other material}

INDONESIA • 4 spec.; Java; SMF $24221 \cdot 3$ spec.; same data as for preceding; ZMB $47683 \cdot 3$ spec.; same data as for preceding; ZMB $47684 \bullet 1$ spec., same data as for preceding; ZMH $17719 \bullet$ 5 spec.; same data as for preceding; ZMH $17720 \bullet 4$ spec.; same data as for preceding; ZMH $17721 \bullet$ 3 spec.; same data as for preceding; ZMH 17722. - West Java 2 spec. (vouchers for van Benthem Jutting 1950); Gora S of Bogor; RMNH $309846 \bullet 7$ spec. (vouchers for Boettger 1890); Bogor, Gunung Salak; $6^{\circ} 44^{\prime}$ S, $106^{\circ} 42^{\prime}$ E; SMF $8917 \cdot 3$ spec. (vouchers for Boettger 1890); same data as for preceding; SMF $24200 \cdot 4$ spec. (vouchers for Boettger 1890); same data as for preceding; SMF $24204 \cdot 2$ spec. (vouchers for Boettger 1890); same data as for preceding; ZMA $407645 \cdot 4 \mathrm{spec}$. (vouchers for van Benthem Jutting 1950); Bogor, Situ Gunung, S slope of Mt Gede, forest; 650' S, 106 $55^{\prime} \mathrm{E} ; 1000 \mathrm{~m}$ a.s.1.; ZMA 407632 • 6 spec.; Gunung Gede; $6^{\circ} 47^{\prime}$ S, $106^{\circ} 59^{\prime}$ E; ZMA 407630 • 2 spec.; same data as for preceding; ZMA 407690 • 9 spec.; Gunung Gede; 647' S, 106 59' E; ca 900 m a.s.l.; SMF 8915 • 16 spec.; Gunung Gede; 6 ${ }^{\circ} 47^{\prime}$ S, $106^{\circ} 59^{\prime}$ E; ca 1200 m a.s.1.; SMF $89136 \bullet 3$ spec.; Gunung Gede; 647’ S, $106^{\circ} 59^{\prime} \mathrm{E}$; ca $1500 \mathrm{~m}$ a.s.1.; ZMB 46035.

\section{Description}

Shell (Figs 5, 12-14; Tables 3-4). Depressed conical, with 5.75-6.5 slightly convex whorls; protoconch almost smooth; teleoconch with fine, irregular wrinkles; apical side with indistinct incised spiral lines, umbilical side with more distinct spiral lines; scaly processes sparse, mainly at periphery; tuberculate around umbilicus; brown or brownish-corneous; body whorl keeled; aperture rounded rhombic; upper insertion of peristome descending; peristome expanded, reflexed and slightly thickened; umbilicus slightly eccentric, wide, comprising $24-32 \%$ of shell diameter, hardly obscured by columellar edge.

Genitalia (Figs 16, 30; Table 4). Atrium short; penis long, distal part cylindrical, with a small globular bulge at proximal end, without penis coecum; membranaceous sheath around distal part of penis connected with epiphallus by tissue; penial retractor runs from diaphragm to proximal part of cylindrical epiphallus; flagellum moderately long, very broad, without node, tapering at its proximal end; vas deferens narrow and long; vagina long; oviduct long, very broad, proximally tapering; peduncle of bursa copulatrix subdivided into a globular distal part, a proximally tapering middle part and a narrower and longer proximal part, ending in an elongate oval bursa beside albumen gland. Right ommatophoral retractor runs between penis and vagina.

\section{Remarks}

The name Helix rotatoria goes back to von dem Busch and is often cited with von dem Busch (in Philippi 1842 in 1842-1845: 10, pl. I.4, fig. 5) as author. However, von dem Busch cited Pfeiffer (1842), who made the name available first (see also remarks under L. winteriana). Pfeiffer (1842) described the species based on specimens from von dem Busch and specimens he got from Winter. The material from Winter was in Pfeiffer's own collection (Pfeiffer 1852 in 1849-1853), which was destroyed during the Second World War (see L. winteriana). A part of the collection of von dem Busch is kept in the

Figs 12-14 (opposite page). Shells of Landouria rotatoria (Pfeiffer, 1842) from Java. 12. West Java, Gunung Gede (holotype of Plectotropis leucomphala Möllendorff, 1897, SMF 8912). 13. West Java, Gunung Salak (holotype of P. leucomphala f. castanea Möllendorff, 1897, SMF 8916). 14. West Java, Gunung Gede (holotype of P. leucomphala f. subfasciata Möllendorff, 1897, SMF 8914). Scale bar: $5 \mathrm{~mm}$. 


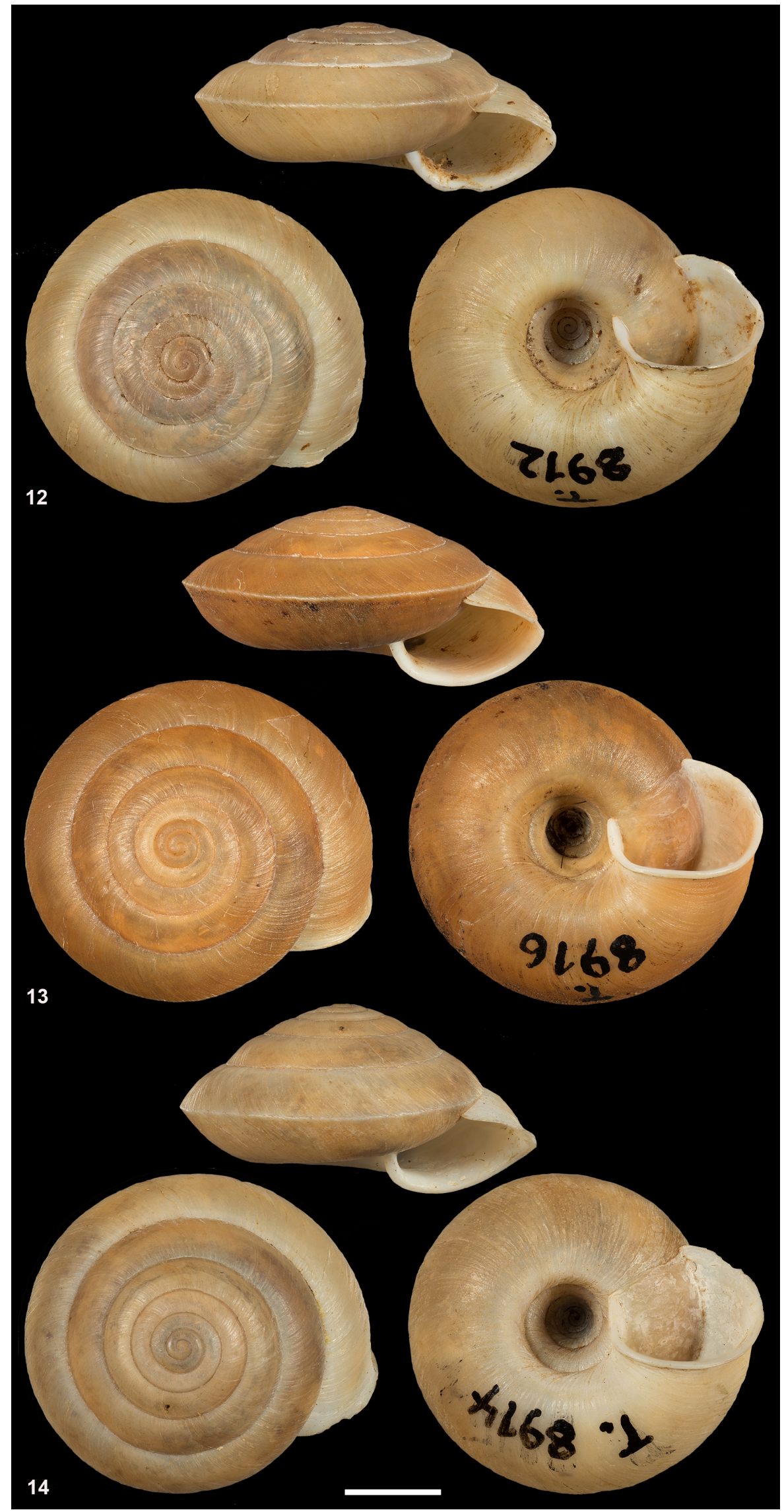



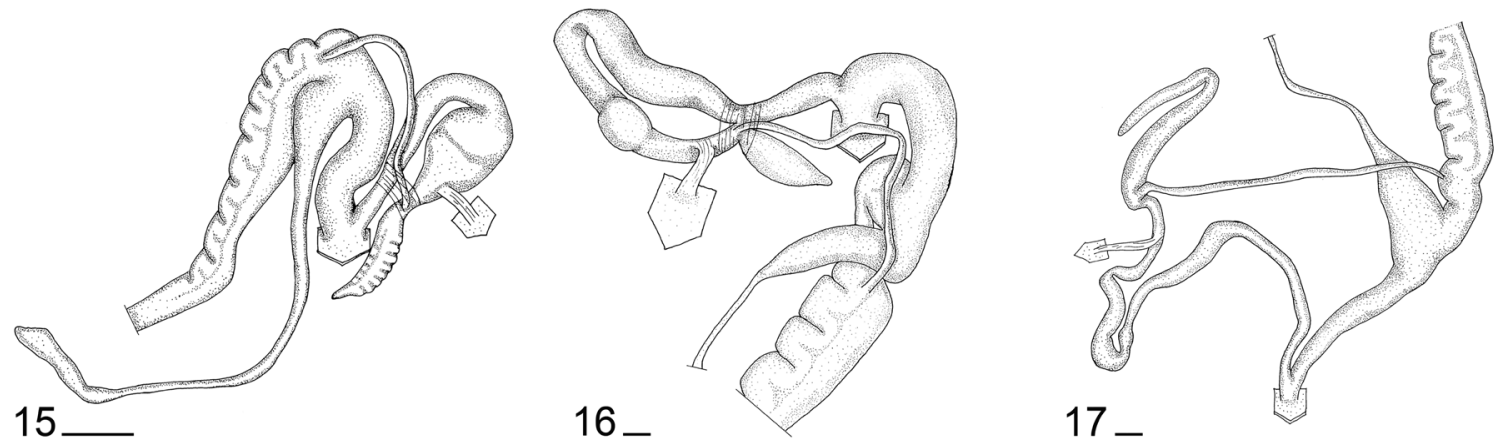

15

16
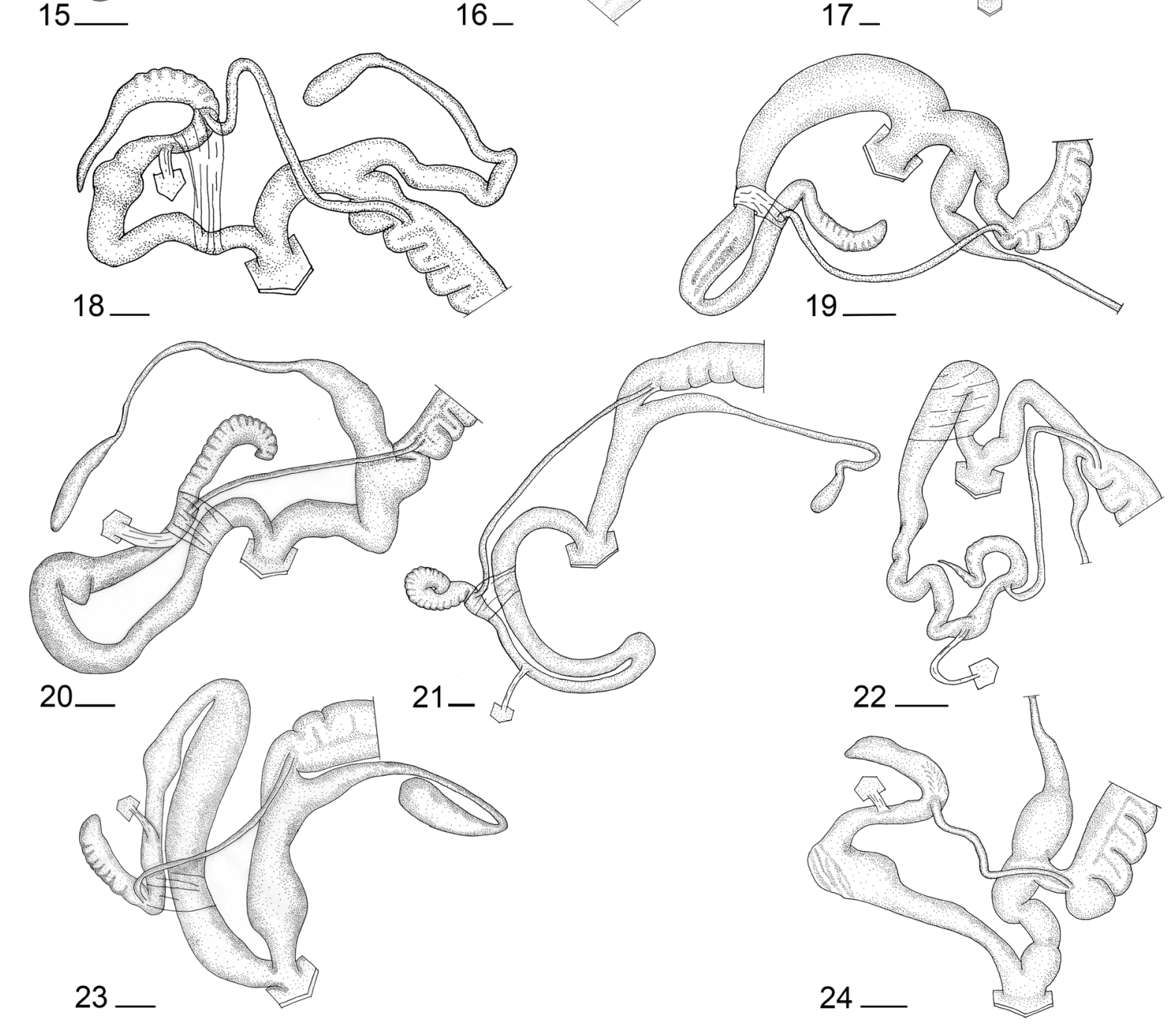

19

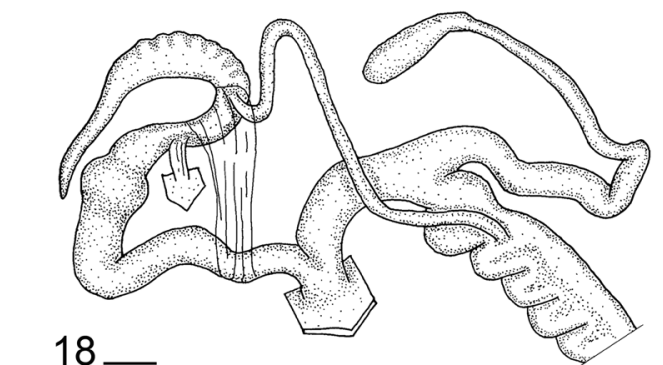

Figs 15-24. Genitalia of species of Landouria from Java. 15. L. winteriana (Pfeiffer, 1842), West Java, Padalarang, Gunung Masigit (neotype, ZMA 376193a). 16. L. rotatoria (Pfeiffer, 1842), West Java, Gunung Salak, 1166 m a.s.l. (neotype, MZB 19205). 17. L. epiplatia (Möllendorff, 1897)?, West Java, Bogor, Gunung Gede (ZMH 79946). 18. L. nusakambangensis sp. nov., Central Java, Nusa Kambangan Island, nature monument Gligir (holotype, ZMA 412298a). 19. L. petrukensis sp. nov., Central Java, Karangbolong, way to Petruk Cave (holotype, MZB 19203). 20. L. tholiformis sp. nov., Yogyakarta, Gunung Kidul, Tepus, near Poktunggal Beach (holotype, MZB 17999). 21. L. abdidalem sp. nov., Yogyakarta, Kulonprogo, Sabrang Kidul (holotype, MZB 17664). 22. L. madurensis sp. nov., East Java, Madura Island, Sampang, Nepa Monkey lowland rainforest (paratype, MZB 19209). 23. L. sewuensis sp. nov., Yogyakarta, Gunung Kidul, Tepus, Mbajo Hill (holotype, MZB 19255). 24. L. sukoliloensis sp. nov., Central Java, Kayen, near Watu Payung (holotype, MZB 19219). Scale bars: $1 \mathrm{~mm}$. 


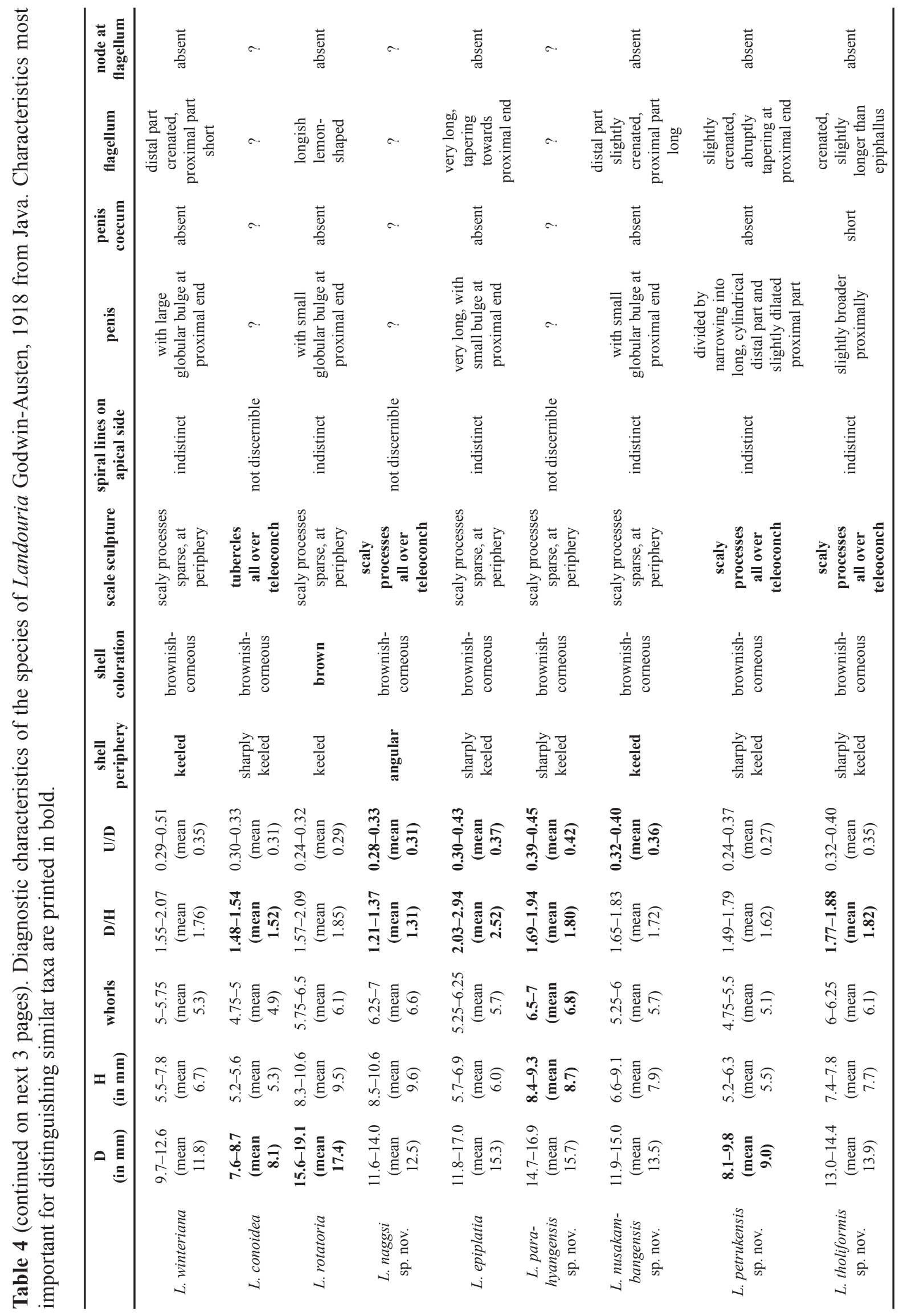




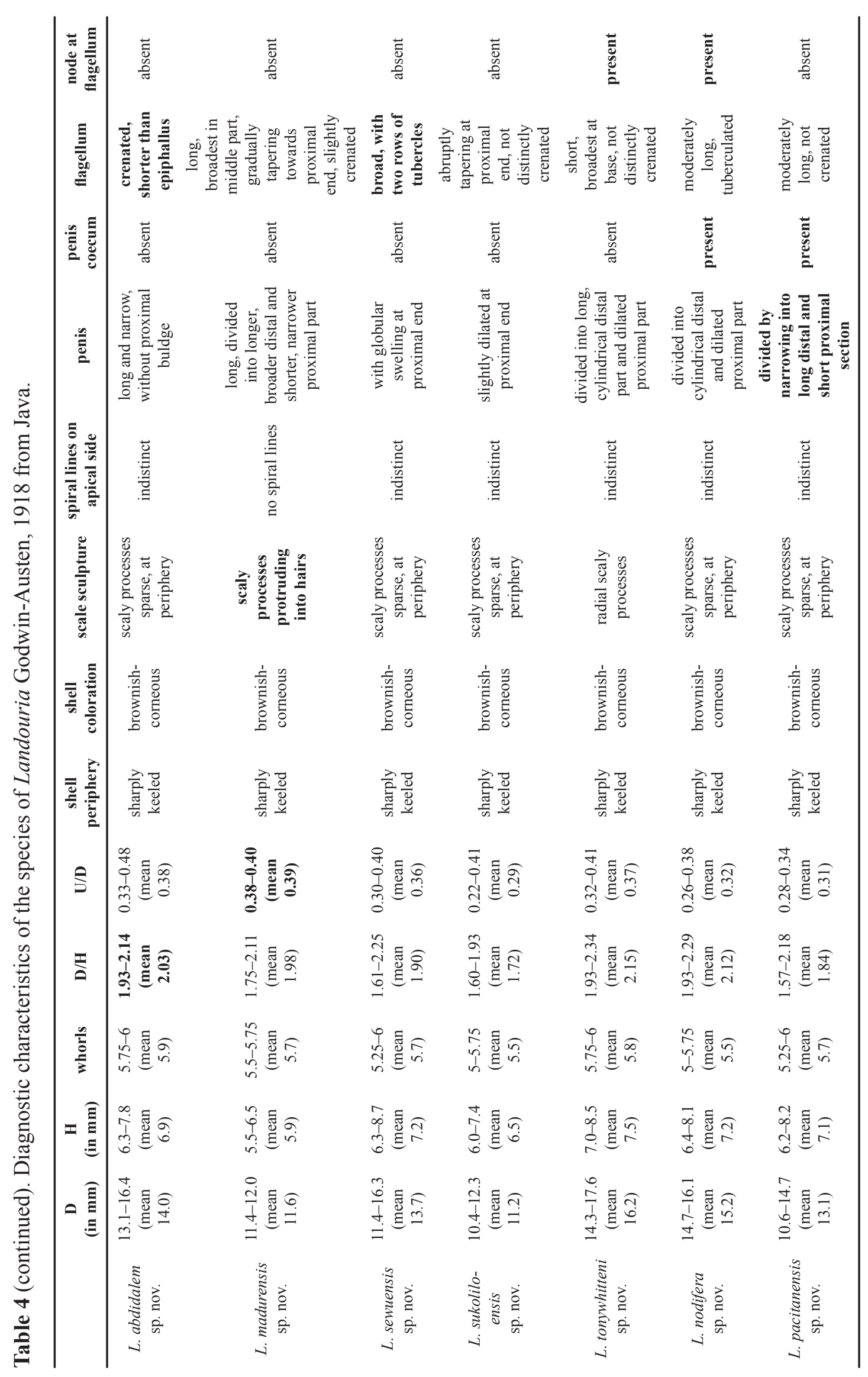




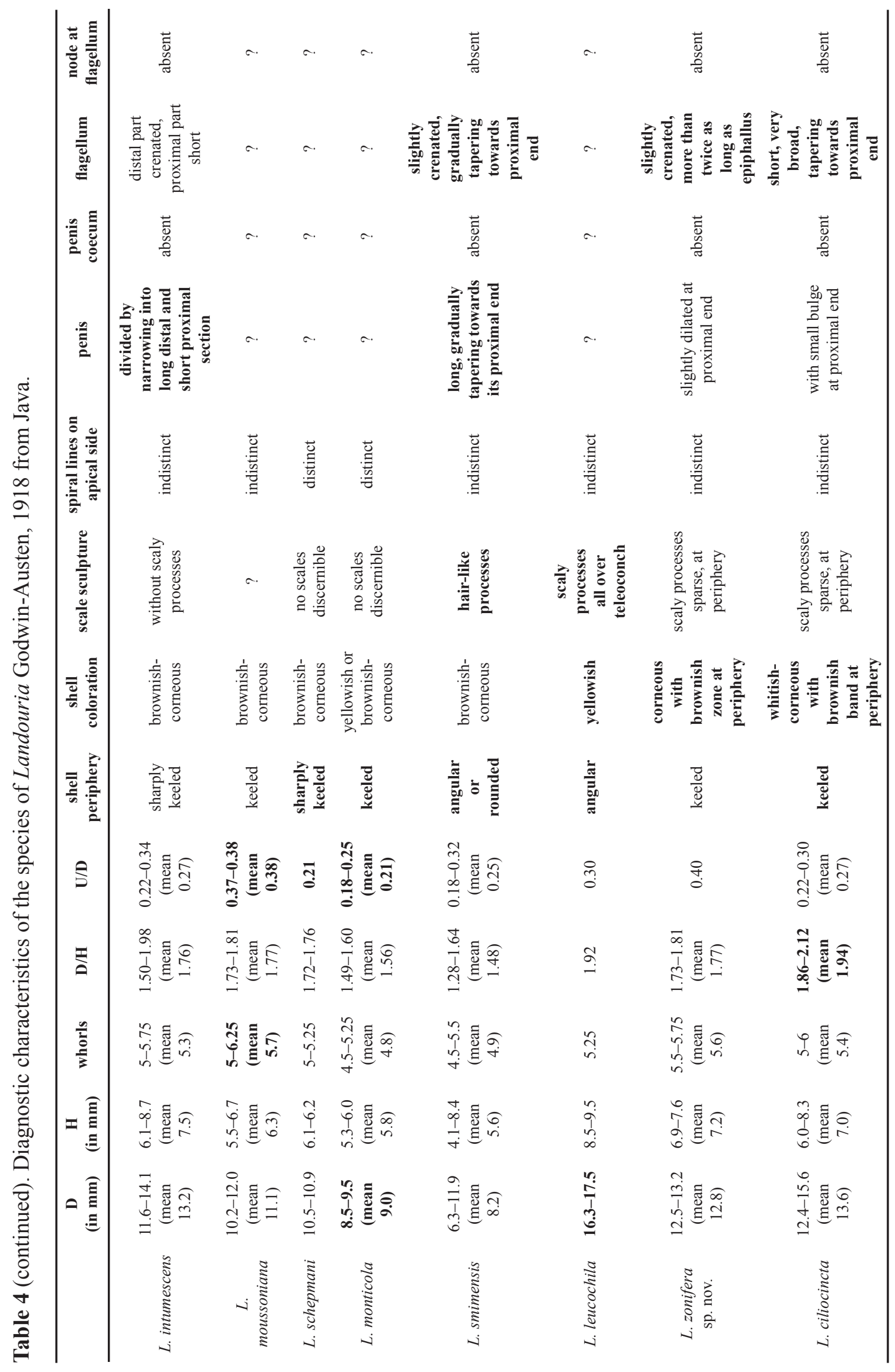




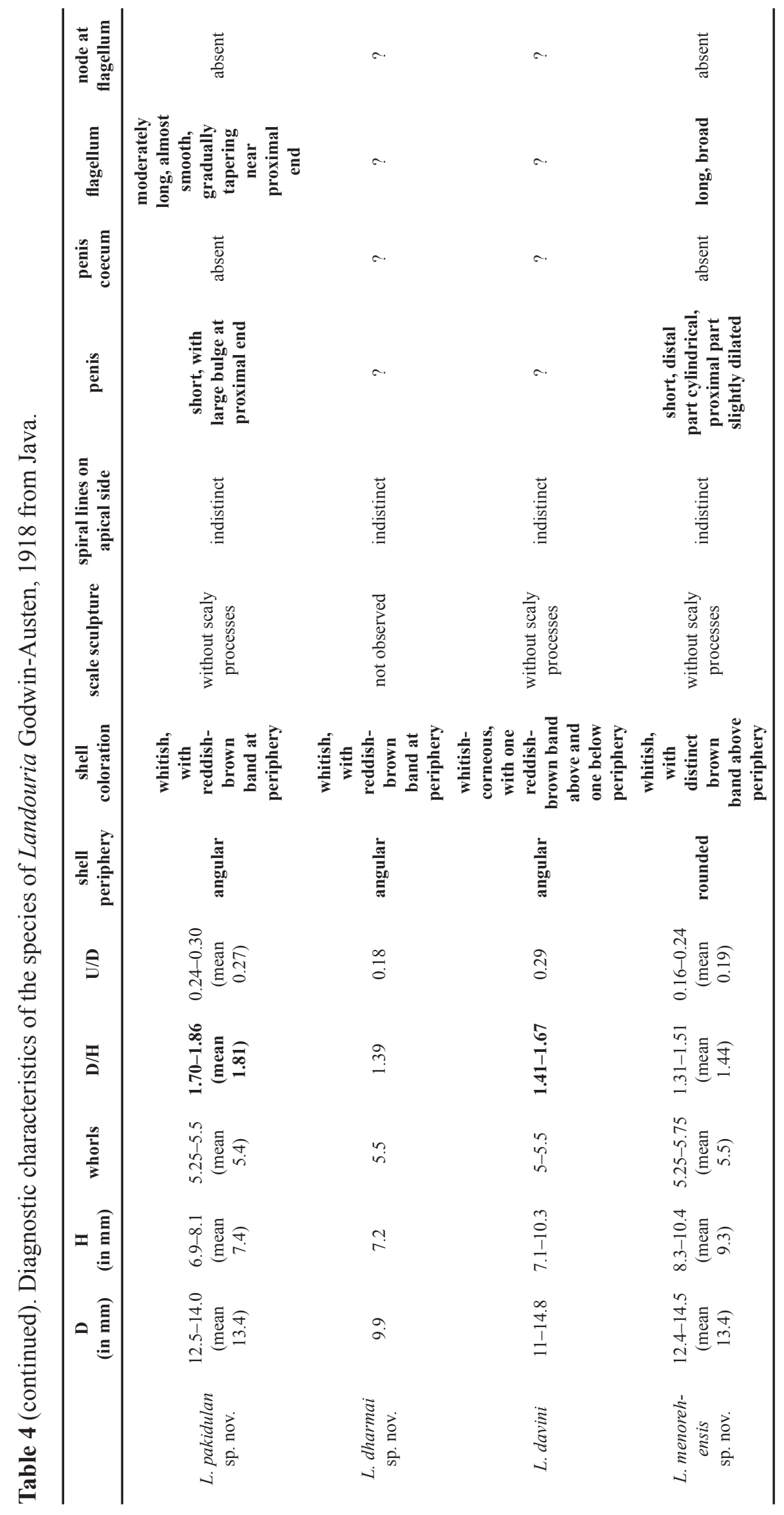


Geosciences Collection of the University of Bremen. However, we could not locate type specimens of $H$. rotatoria in this collection or in other museums (e.g., SMF, ZMH). Thus, a neotype must be designated to clarify the taxonomic status and the type locality of $H$. rotatoria.

According to the original description of Pfeiffer (1842), H. rotatoria is a brown, sharply keeled, large species with a diameter of $16 \mathrm{~mm}$. There are several sharply keeled species in Java and some reach a diameter of $16 \mathrm{~mm}$. The figures of Pfeiffer (1851 in 1849-1853: pl. 95, figs 3-5) show a specimen with a rather high spire (D/H ratio 1.82). The species that best fits the figure occurs on Gunung Salak and Gunung Gede south of Bogor in West Java (compare the shell of the holotype of P. leucomphala Moellendorff, 1897 (Fig. 12) with Pfeiffer 1851 in 1849-1853: pl. 95, figs 3-5). Thus, we designate an anatomically examined specimen from Gunung Salak in West Java (MZB 19205; Fig. 5) as the neotype of Helix rotatoria Pfeiffer, 1842.

The same species was later described by Möllendorff (1897) as Plectotropis leucomphala with two colour varieties, P. leucomphala f. subfasciata and P. leucomphala f. castanea. Plectotropis leucomphala was synonymized with $L$. rotatoria by van Benthem Jutting (1950).

Landouria rotatoria differs from $L$. winteriana (Figs 2-3), which also occurs on Gunung Salak, and from L. epiplatia (Figs 26-28, 31), which also occurs on Gunung Gede, in the larger, more intensively brownish coloured shell and an externally smooth, longish, lemon-shaped flagellum.

\title{
Distribution
}

Landouria rotatoria is endemic to Gunung Salak and Gunung Gede south of Bogor in West Java (Fig. 54). It has also been recorded from other Sunda Islands, as well as the Philippines (Martens 1867; Rensch 1932, 1934; van Benthem Jutting 1941, 1950; Vermeulen \& Whitten 1998), but these records refer to other species.

\author{
Landouria naggsi sp. nov. \\ urn:1sid:zoobank.org:act:4EF9E468-DDEF-460D-939C-7D9C86254CF4
}

Figs 25, 52; Tables 3-4

\section{Diagnosis}

Landouria naggsi sp. nov. is characterized by an angular, tightly coiled, high conical shell with scales all over the teleoconch.

\section{Etymology}

Landouria naggsi sp. nov. is named in honour of the British malacologist Fred Naggs, who brought the authors of this study together.

\section{Material examined}

\section{Holotype}

INDONESIA - holotype; West Java, Cibitung, near Cikaso Waterfall, teak agroforestry; $7^{\circ} 21^{\prime} 41^{\prime \prime} \mathrm{S}$, $106^{\circ} 37^{\prime} 10^{\prime \prime}$ E; 47 m a.s.1.; 5 Jul. 2015; A.S. Nurinsiyah and T. Setiadi leg.; D = $11.8 \mathrm{~mm}, \mathrm{H}=9.8 \mathrm{~mm}$; MZB 20424.

\section{Paratypes}

INDONESIA - West Java -1 spec.; Cibitung, near Cikaso Waterfall, riparian (high shrubs); $7^{\circ} 21^{\prime} 40^{\prime \prime} \mathrm{S}, 106^{\circ} 37^{\prime} 05^{\prime \prime} \mathrm{E}$; $61 \mathrm{~m}$ a.s.1.; MZB 20426 • 1 spec.; same data as for preceding; ZMH 


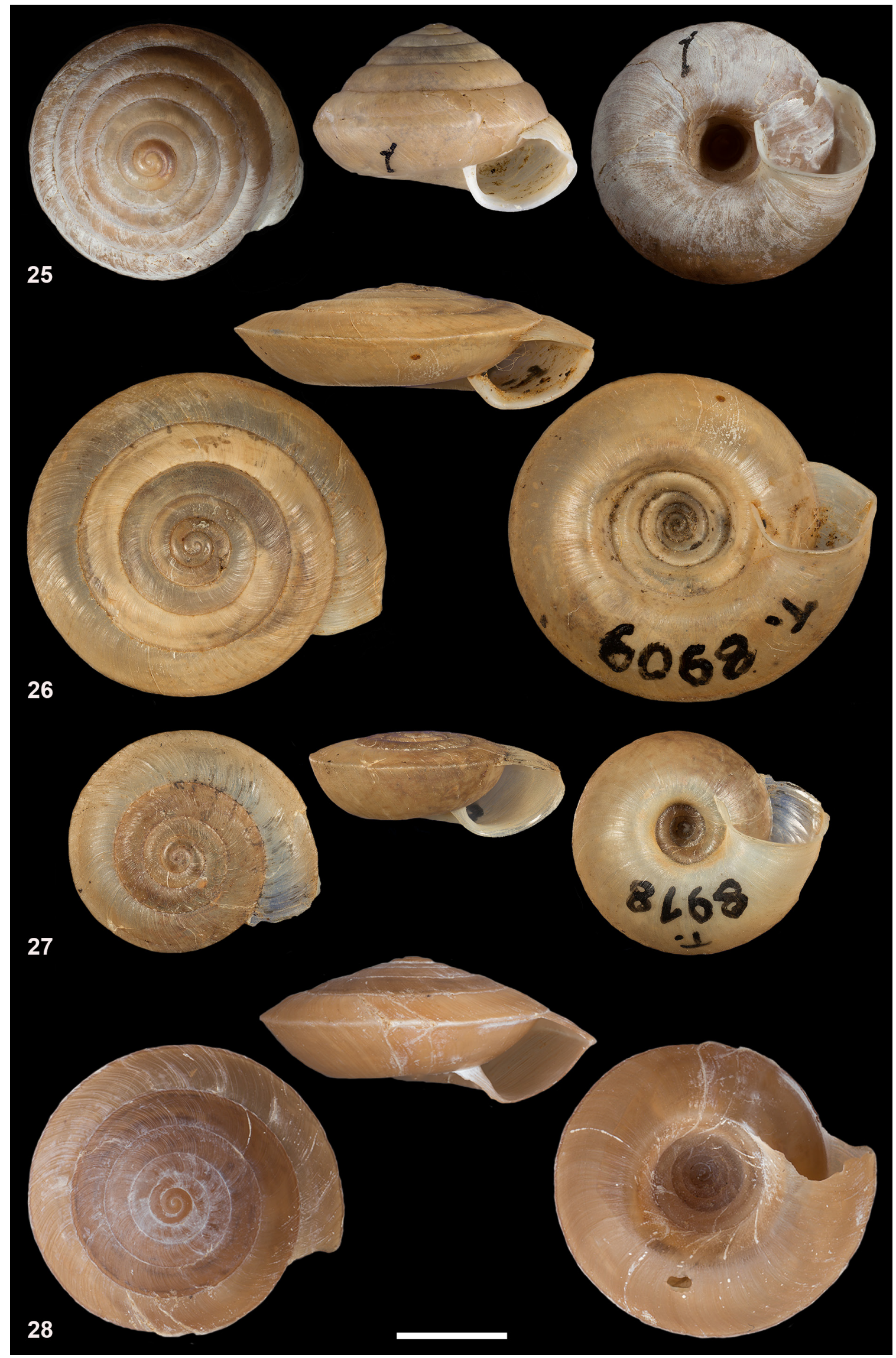


148184 • 3 spec.; same data as for holotype; MZB 20425 • 2 spec.; same data as for holotype; ZMH 148183.

\section{Description}

SHELl (Fig. 25; Tables 3-4). Conical, with 6.25-7 slightly convex whorls; protoconch almost smooth; teleoconch with irregular wrinkles; incised spiral lines not discernible; scales all over teleoconch; tuberculate around umbilicus; brownish-corneous; body whorl angular; aperture oval; upper insertion of peristome slightly descending; peristome expanded, reflexed and slightly thickened; umbilicus hardly eccentric, wide, comprising $28-33 \%$ of shell diameter, hardly obscured by columellar edge.

Genitalia. Unknown.

\section{Remarks}

Landouria naggsi sp. nov. resembles L. parahyangensis sp. nov. (Fig. 48), from which it differs in the smaller (D 11.6-14.0 mm vs 14.7-16.9 mm in L. parahyangensis sp. nov.), higher conical (D/H: 1.211.37 vs $1.69-1.94$ in $L$. parahyangensis sp. nov.), less sharply keeled shell with a narrower umbilicus (U/D 0.28-0.33 vs 0.39-0.45 in L. parahyangensis sp. nov.) and in having scales all over the teleoconch.

With regard to the conical shell form L. naggsi sp. nov. resembles L. conoidea (Leschke, 1914) (Fig. 4). For differences, see remarks under that species.

\section{Distribution}

Landouria naggsi sp. nov. is known only from the surroundings of Cikaso Waterfall in Sukabumi Regency (Fig. 52).

Landouria epiplatia (Möllendorff, 1897)

Figs 17, 26-28, 31, 55; Tables 3-4

Plectotropis epiplatia Möllendorff, 1897: 67 (“Java”; more exactly (Zilch 1966): “W-Java: Djampang $\left.\left(2000^{\prime}\right) "\right)$.

Plectotropis trichotrochium Möllendorff, 1897: 67 ("Java", more exactly (Zilch 1966): "W-Java: Djampang (2000'), s. Susabuni [sic]"). Syn. nov.

Landouria epiplatia - van Benthem Jutting 1950: 466, fig. 82. — Zilch 1966: 293, pl. 7, fig. 2.

Landouria rotatoria - van Benthem Jutting 1950: 461 (in part) . - Zilch 1966: 294, pl. 7, fig. 6 (in part). (not Pfeiffer, 1842)

\section{Diagnosis}

Landouria epiplatia is characterized by a brownish, sharply keeled, almost disc-like shell with an only slightly elevated spire and a very wide umbilicus.

Figs 25-28 (opposite page). Shells of species of Landouria from Java. 25. L. naggsi sp. nov., West Java, Cibitung, near Cikaso Waterfall (holotype, MZB 20424). 26. L. epiplatia (Möllendorff, 1897), West Java, Jampang (lectotype of Plectotropis epiplatia, SMF 8909). 27. L. epiplatia, West Java, Jampang (lectotype of Plectotropis trichotrochium Möllendorff, 1897, SMF 8918). 28. L. epiplatia?, West Java, Bogor, Gunung Gede (ZMH 79946). Scale bar: 5 mm. 


\section{Material examined}

Type material

INDONESIA - West Java - lectotype of Plectotropis epiplatia (designated by Zilch 1966); Jampang; $7^{\circ} 03^{\prime} \mathrm{S}, 106^{\circ} 48^{\prime} \mathrm{E}$; ca $600 \mathrm{~m}$ a.s.1.; SMF $8909 \cdot 1$ paralectotype of Plectotropis epiplatia; same data as for preceding; SMF 8910 lectotype of Plectotropis trichotrochium; same data as for preceding; SMF 8918.

\section{Other material}

INDONESIA • 1 spec.; Java; ZMB 47682. - West Java 4 spec.; 5 km from Palabuhan Ratu Bay; $7^{\circ} 00^{\prime} \mathrm{S}, 106^{\circ} 35^{\prime}$ E; SMF $96542 \bullet 2$ spec.; Jampang; $7^{\circ} 03^{\prime} \mathrm{S}, 106^{\circ} 48^{\prime}$ E; ca 600 m a.s.1.; SMF $8911 \bullet$

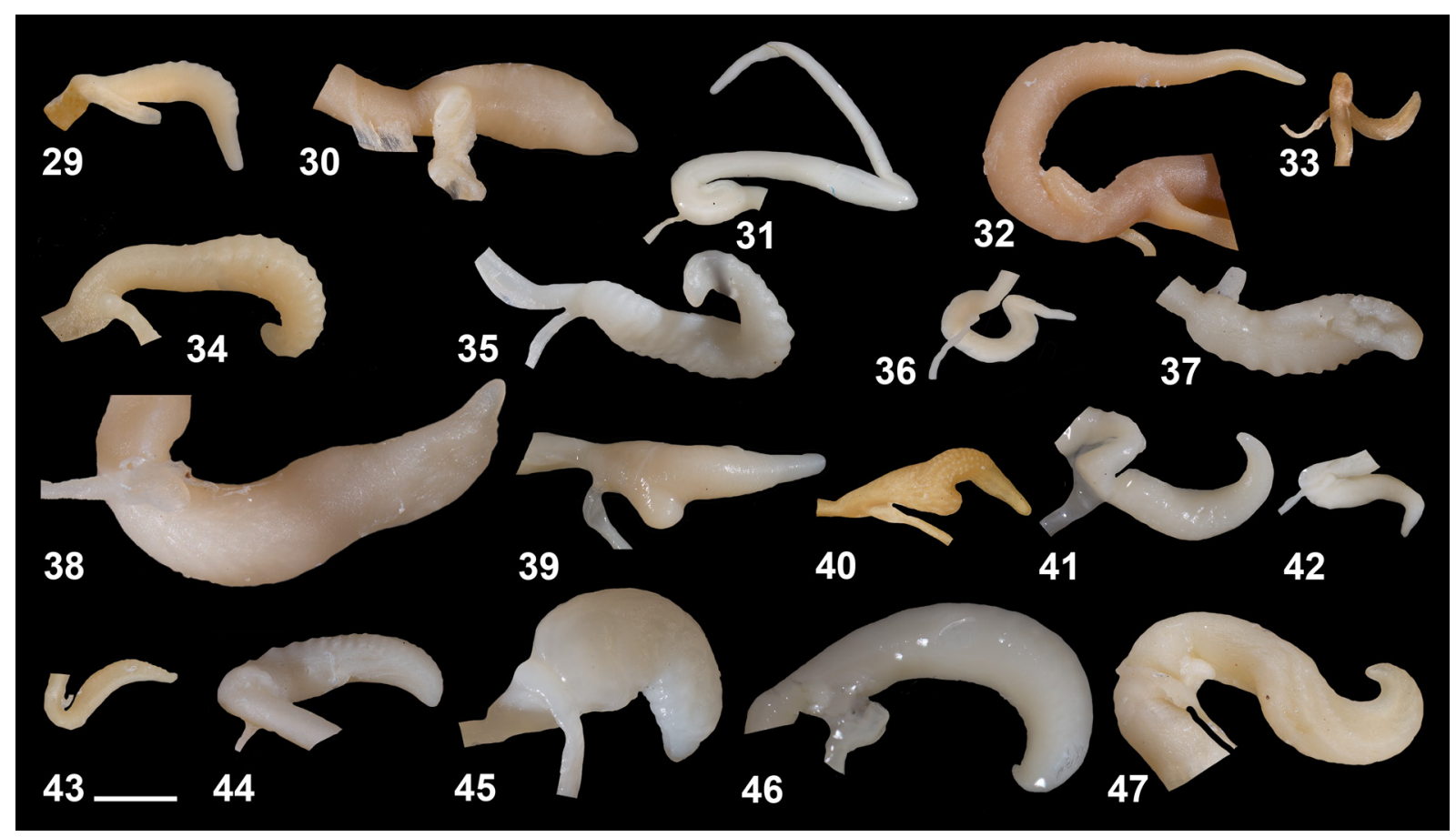

Figs 29-47. Flagella of species of Landouria from Java. 29. L. winteriana (Pfeiffer, 1842), West Java, Padalarang, Gunung Masigit (neotype, ZMA 376193a). 30. L. rotatoria (Pfeiffer, 1842), West Java, Gunung Salak, 1166 m a.s.1. (neotype, MZB 19205). 31. L. epiplatia (Möllendorff, 1897)?, West Java, Bogor, Gunung Gede (ZMH 79946). 32. L. nusakambangensis sp. nov., Central Java, Nusa Kambangan Island, nature monument Gligir (holotype, ZMA 412298a). 33. L. petrukensis sp. nov., Central Java, Karangbolong, way to Petruk Cave (holotype, MZB 19203). 34. L. tholiformis sp. nov., Yogyakarta, Gunung Kidul, Tepus, near Poktunggal Beach (holotype, MZB 17999). 35. L. abdidalem sp. nov., Yogyakarta, Kulonprogo, Sabrang Kidul (holotype, MZB 17664). 36. L. madurensis sp. nov., East Java, Madura Island, Sampang, Nepa Monkey lowland rainforest (paratype, MZB 19209). 37. L. sewuensis sp. nov., Yogyakarta, Gunung Kidul, Tepus, Mbajo Hill (holotype, MZB 19255). 38. L. sukoliloensis sp. nov., Central Java, Kayen, near Watu Payung (holotype, MZB 19219). 39. L. tonywhitteni sp. nov., Central Java, Taruman Village, near Sendang Sedayu (holotype, MZB 19214). 40. L. nodifera sp. nov., Central Java, Larangan Village, Lanang Spring (holotype, MZB 19227). 41. L. pacitanensis sp. nov., East Java, Pacitan, near Srau Beach (MZB 19231). 42. L. intumescens (Martens, 1867), East Java, Guwoterus (MZB 19207). 43. L. smimensis (Mousson, 1848), East Java, Tosari (ZMA 390881). 44. L. zonifera sp. nov., Yogyakarta, Kulonprogo, Sibolong (holotype, MZB 17727). 45. L. ciliocincta (Möllendorff, 1897), East Java, Malang, Kondangmerak (MZB 19211). 46. L. pakidulan sp. nov., Central Java, Nusa Kambangan Island, nature monument Gligir (holotype, ZMA 417011a). 47. L. menorehensis sp. nov., Yogyakarta, Kulonprogo, Sibolong (holotype, MZB 19201). Scale bar: $1 \mathrm{~mm}$. 
2 spec. (vouchers for van Benthem Jutting 1950); Sukabumi; 6 ${ }^{\circ} 55^{\prime}$ S, $106^{\circ} 55^{\prime}$ E; ZMA 407610 - 1 spec. (det. anat.; see remarks); Bogor, Gunung Gede; 646' S, 10659' E; ZMH 79946.

\section{Description}

SHELl (Figs 26-28; Tables 3-4). Almost disc-like, with 5.25-6.25 hardly convex whorls; protoconch almost smooth; teleoconch with irregular wrinkles; apical side with indistinct incised spiral lines, umbilical side with more distinct spiral lines; scaly processes sparse, mainly at periphery; tuberculate around umbilicus; brownish-corneous; body whorl sharply keeled; aperture rounded rhombic; upper insertion of peristome slightly descending; peristome expanded, slightly reflexed and slightly thickened; umbilicus almost concentric, very wide, comprising $30-43 \%$ of shell diameter, hardly obscured by columellar edge.

Genitalia (Figs 17, 31; Table 4). Atrium short; penis very long, with a small bulge at proximal end containing a short penis papilla; without penis coecum; membranaceous sheath around distal part of penis connected with epiphallus by tissue; penial retractor runs from diaphragm to proximal part of cylindrical epiphallus; flagellum very long, broadest in its middle part, without node, tapering towards its proximal end; vas deferens narrow and long; vagina long; oviduct short, broad; peduncle of bursa copulatrix subdivided into a broader, abruptly tapering distal part and a narrower, longer proximal part. Right ommatophoral retractor runs between penis and vagina.

\section{Remarks}

Landouria epiplatia was described from Jampang in West Java. However, there are several places called Jampang in West Java, two among them being more important ones: Jampang Kulon and Jampang Tengah in Sukabumi Regency. Jampang Kulon is at ca $300 \mathrm{~m}$ a.s.l., Jampang Tengah is at ca $500 \mathrm{~m}$ a.s.l. and closer to Sukabumi, the locality given by van Benthem Jutting (1950). Thus, we suppose that Jampang Tengah is the type locality.

Plectotropis trichotrochium Möllendorff, 1897 (Fig. 27) was synonymized with L. rotatoria by van Benthem Jutting (1950) and Zilch (1966). However, in contrast to L. rotatoria, the spire of the single specimen on which $P$. trichotrochium was based is hardly elevated. We suppose that the holotype of P. trichotrochium is a subadult specimen of $L$. epiplatia that formed a slightly expanded peristome at a premature stage. The specimen is furthermore characterized by stronger radial wrinkles and more distinct incised spiral lines than is usual in L. epiplatia. Nevertheless, its occurrence at the type locality of L. epiplatia also indicates that it is an aberrant specimen of that species. As first revisers (ICZN 1999: Art. 24), we determine precedence of the name Plectotropis epiplatia Möllendorff, 1897 over Plectotropis trichotrochium Möllendorff, 1897.

The description of the genitalia is based on a single subadult specimen from Gunung Gede (ZMH 79946; Figs 17, 31), which differs from typical L. epiplatia in the slightly tighter coiled, more elevated spire. The available material is insufficient to decide whether this specimen falls within the variability of L. epiplatia or represents a distinct species.

\section{Distribution}

Landouria epiplatia is known from the northern part of Sukabumi Regency in West Java and its range might extend to Gunung Gede (Fig. 55). 
Landouria parahyangensis $\mathrm{sp}$. nov.

urn:lsid:zoobank.org:act:41EEB2F2-7781-4D19-9F62-34316F374749

Figs 48, 54; Tables 3-4

\section{Diagnosis}

Landouria parahyangensis sp. nov. is characterized by a sharply keeled, tightly coiled conical shell with a very wide umbilicus.

\section{Etymology}

Landouria parahyangensis sp. nov. is named after its occurrence in the mountainous region Parahyangan. In the Sundanese language, the name 'Parahyangan' means 'the abode of hyangs (gods)', which were supposed to reside in the mountain tops.

\section{Material examined}

\section{Holotype}

INDONESIA • holotype; West Java, Karangnunggal, surroundings of Pasir Taraje Cave, agroforestry; 7³9'58" S, 108 07'01" E; 243 m a.s.l.; 4 Jul. 2014; A.S. Nurinsiyah and T. Setiadi leg.; D = $15.0 \mathrm{~mm}$, $\mathrm{H}=8.4 \mathrm{~mm} ;$ MZB 20429 .

\section{Paratypes}

INDONESIA - West Java 3 spec.; Karangnunggal, surroundings of Kiara Jenggot Cave, agroforestry; 7³9'37" S, 10806'55" E; 234 m a.s.1.; MZB 20431 • 10 spec.; Karangnunggal, near Sela Dower Cave, agroforestry and bamboo; $7^{\circ} 40^{\prime} 00^{\prime \prime} \mathrm{S}, 108^{\circ} 07^{\prime} 00^{\prime \prime} \mathrm{E} ; 237 \mathrm{~m}$ a.s.1.; MZB $20428 \cdot 5$ spec.; same data as for preceding; ZMH $148186 \bullet 3$ spec.; same data as for holotype; MZB $20430 \bullet 3 \mathrm{spec}$.; same data as for holotype; ZMH $148187 \cdot 1 \mathrm{spec}$.; Karangnunggal, surroundings of Liang Cai, agroforestry and shrubs; $7^{\circ} 40^{\prime} 01^{\prime \prime}$ S, $108^{\circ} 07^{\prime} 03^{\prime \prime}$ E; 237 m a.s.1.; ZMH $148185 \bullet 1$ spec.; Tasikmalaya, Karangnunggal, mouth of Bojong Cave, agroforestry; 740'12" S, 108 07'12" E; 240 m a.s.1.; MZB 20427.

\section{Description}

SHELl (Fig. 48; Tables 3-4). Conical, with 6.5-7 hardly convex whorls; protoconch almost smooth; teleoconch with fine, irregular wrinkles; without distinct incised spiral lines; scaly processes sparse, only at periphery; tuberculate around umbilicus; brownish-corneous; body whorl sharply keeled; aperture rounded rhombic; upper insertion of peristome slightly descending; peristome expanded, reflexed and slightly thickened; umbilicus hardly eccentric, wide, comprising $39-45 \%$ of shell diameter, hardly obscured by columellar edge.

Genitalia. Unknown.

\section{Remarks}

Landouria parahyangensis sp. nov. resembles L. epiplatia (Figs 26-28) with regard to the wide umbilicus. It differs from L. epiplatia in the less sharply keeled, higher, conical shell (H 8.4-9.3 mm vs $5.7-6.9 \mathrm{~mm}$ in L. epiplatia; D/H 1.69-1.94 vs 2.03-2.94 in L. epiplatia) with more whorls (6.5-7

Figs 48-51 (opposite page). Shells of species of Landouria from Java. 48. L. parahyangensis sp. nov., West Java, Karangnunggal, surroundings of Pasir Taraje Cave (holotype, MZB 20429). 49. L. nusakambangensis sp. nov., Central Java, Nusa Kambangan Island, nature monument Gligir (holotype, ZMA 412298a). 50. L. petrukensis sp. nov., Central Java, Karangbolong, way to Petruk Cave (holotype, MZB 19203). 51. L. tholiformis sp. nov., Yogyakarta, Gunung Kidul, Tepus, near Poktunggal Beach (holotype, MZB 17999). Scale bar: 5 mm. 
NURINSIYAH A.S. et al., Revision of Landouria from Java

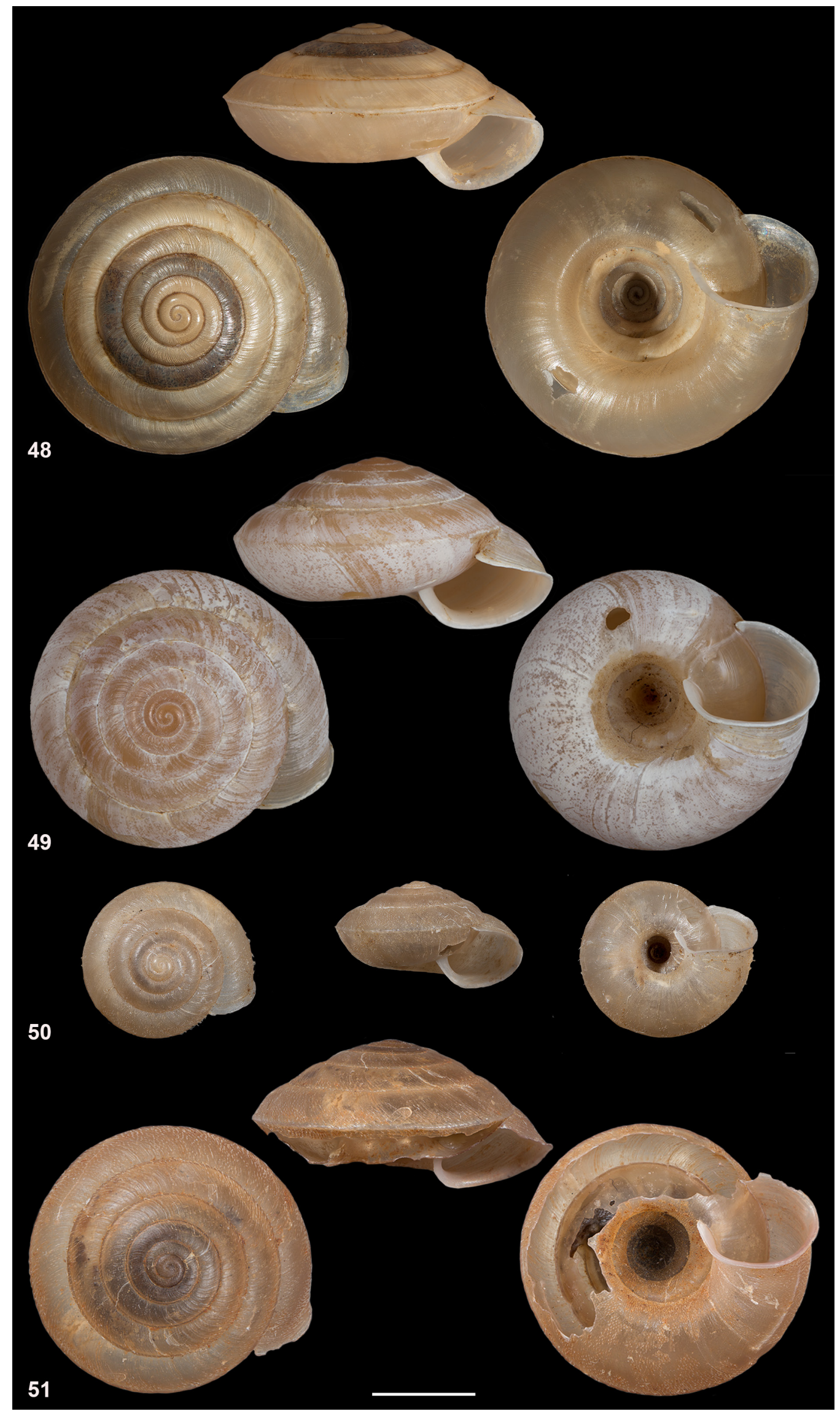


vs 5.25-6.25 in L. epiplatia). Landouria parahyangensis sp. nov. also resembles L. naggsi sp. nov. (Fig. 25). For differences, see remarks under that species.

\section{Distribution}

Landouria parahyangensis sp. nov. is endemic to the Karangnunggal karst area in West Java (Fig. 54).

Landouria nusakambangensis sp. nov. urn:1sid:zoobank.org:act:3F1A3273-1ECA-4D55-B350-AC9FE593D6AD

Figs 18, 32, 49, 52; Tables 3-4

Landouria rotatoria - van Benthem Jutting 1941: 310 (in part). (not Pfeiffer, 1842)

\section{Diagnosis}

Landouria nusakambangensis sp. nov. is characterized by a brownish-corneous, keeled shell, a penis with a small globular bulge at the proximal end and a flagellum consisting of a crenated distal part and a long tapering proximal part.

\section{Etymology}

Landouria nusakambangensis sp. nov. is named after its occurrence on Nusa Kambangan Island.

\section{Material examined}

\section{Holotype}

INDONESIA • holotype (det. anat.); Central Java, Nusa Kambangan Island, Nature Monument Gligir; 7²4' S, 10853' E; 15-16 Feb. 1927; P.F. Franck leg.; D = 14.9 mm, H = 9.1 mm; ZMA 412298a.

\section{Paratypes}

INDONESIA -7 spec. (vouchers for van Benthem Jutting 1941); same data as for holotype; ZMA $412298 \cdot 16$ spec. (vouchers for van Benthem Jutting 1941); same data as for holotype; ZMA 410726 - 6 spec. (vouchers for van Benthem Jutting 1941); Nusa Kambangan Island; 744' S, 108 $53^{\prime}$ E; ZMA 407622.

\section{Description}

SHELl (Fig. 49; Tables 3-4). Depressed conical, with 5.25-6 hardly convex whorls; protoconch almost smooth; teleoconch with fine, irregular wrinkles; apical side with indistinct incised spiral lines, umbilical side with more distinct spiral lines and a granular sculpture; scaly processes sparse, mainly at periphery; tuberculate around umbilicus; brownish-corneous; body whorl keeled; aperture rounded rhombic; upper insertion of peristome descending; peristome expanded, reflexed and thickened; umbilicus slightly eccentric, wide, comprising $32-40 \%$ of shell diameter, hardly obscured by columellar edge.

Figs 52-55 (opposite page). Distribution of species of Landouria in Java (10' grid). 52. L. winteriana (Pfeiffer, 1842), L. naggsi sp. nov., L. nusakambangensis sp. nov., L. petrukensis sp. nov., L. tholiformis sp. nov., L. tonywhitteni sp. nov., L. smimensis (Mousson, 1848). 53. L. conoidea (Leschke, 1914), L. abdidalem sp. nov., L. sewuensis sp. nov., L. sukoliloensis sp. nov., L. pacitanensis sp. nov., L. intumescens (Martens, 1867), L. madurensis sp. nov. 54. L. rotatoria (Pfeiffer, 1842), L. nodifera sp. nov., L. parahyangensis sp. nov., L. schepmani (Möllendorff, 1897), L. zonifera sp. nov., L. ciliocincta (Möllendorff, 1897), L. pakidulan sp. nov. 55. L. epiplatia (Möllendorff, 1897), L. monticola van Benthem Jutting, 1950, L. davini Dharma, 2015, L. menorehensis sp. nov., L. dharmai sp. nov., L. moussoniana (Martens, 1867). 
NURINSIYAH A.S. et al., Revision of Landouria from Java
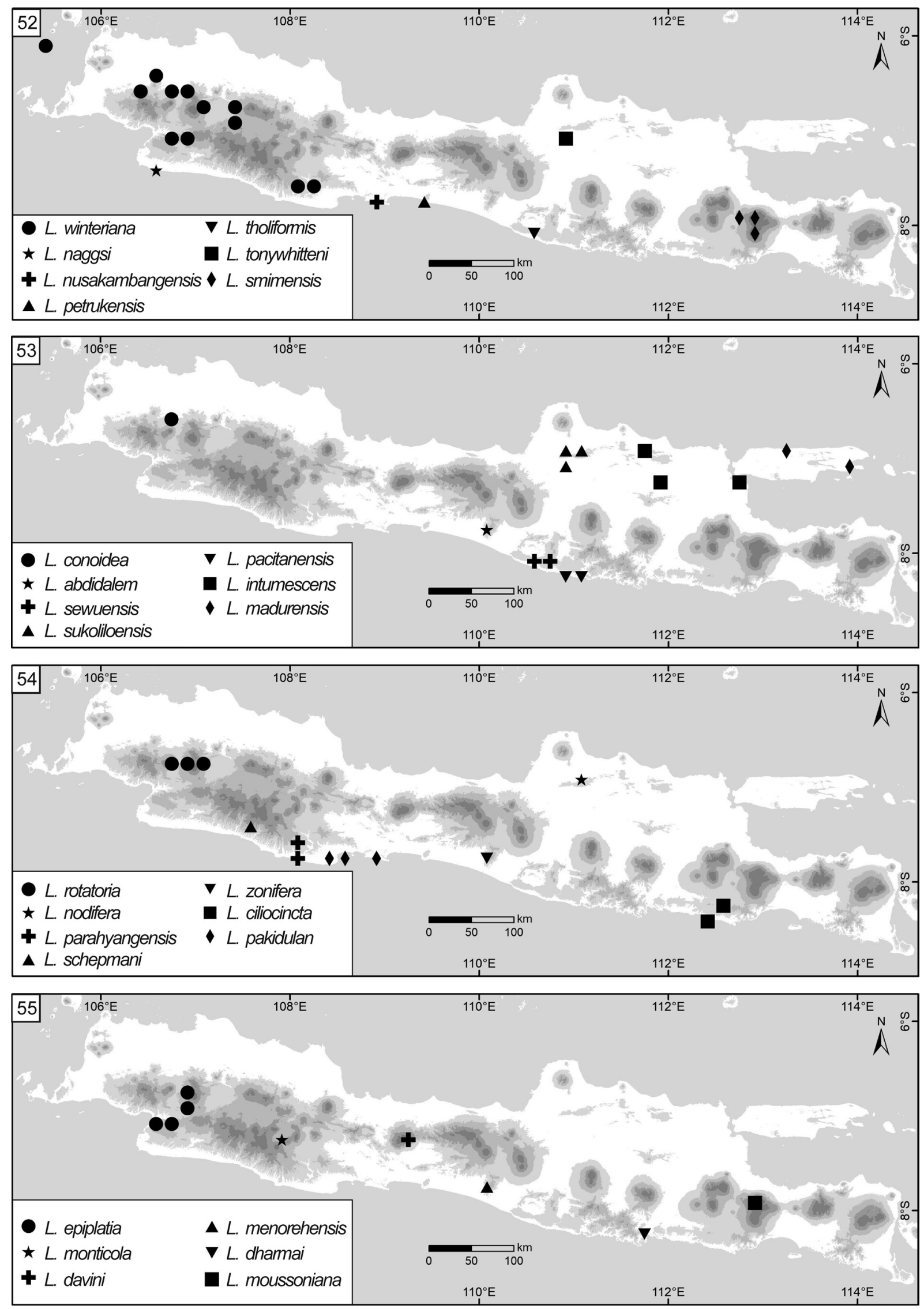
Genitalia (Figs 18, 32; Table 4). Atrium short; penis long, becoming broader proximally; with a small globular bulge at proximal end; without penis coecum; membranaceous sheath around distal part of penis connected with proximal part of epiphallus by tissue; penial retractor runs from diaphragm to proximal part of cylindrical epiphallus; flagellum moderately long, broader distal part usually slightly crenated, proximal part tapering towards its proximal end, without node; vas deferens narrow and long; vagina long; oviduct short, broad, proximally tapering; peduncle of bursa copulatrix subdivided into a broader, cylindrical distal part and a narrower, longer proximal part, ending in an elongate oval bursa beside albumen gland. Right ommatophoral retractor runs between penis and vagina.

\section{Remarks}

Landouria nusakambangensis sp. nov. resembles L. winteriana, from which it differs in the slightly more distinct keel, the smaller globular bulge at the proximal end of the penis and a longer tapering proximal part of the flagellum. In shell shape and the keeled body whorl, L. nusakambangensis sp. nov. also resembles $L$. rotatoria, from which it differs in the smaller shell (D 11.9-15.0 mm vs $15.6-19.1 \mathrm{~mm}$ in L. rotatoria) with a wider umbilicus (U/D $0.32-0.40$ vs $0.24-0.32$ in L. rotatoria).

Landouria nusakambangensis sp. nov. occurs sympatrically with L. pakidulan sp. nov. (Fig. 85), from which it can easily be distinguished by the brownish, keeled shell.

\section{Distribution}

Landouria nusakambangensis sp. nov. is known only from Nusa Kambangan Island (Fig. 52).

Landouria petrukensis sp. nov. urn:1sid:zoobank.org:act:32D7E6E9-561F-4E05-95D2-66295BFD6C5E

Figs 19, 33, 50, 52; Tables 3-4

\section{Diagnosis}

Landouria petrukensis sp. nov. is characterized by a rather high, sharply keeled shell with small scales all over the teleoconch, a penis that is divided into a long, broad, cylindrical distal part and a slightly dilated proximal part by a narrow point and a flagellum, which is broadest near its proximal end.

\section{Etymology}

Landouria petrukensis sp. nov. is named after its occurrence in the surroundings of Petruk Cave in Karangbolong.

\section{Material examined}

\section{Holotype}

INDONESIA • holotype (det. anat.); Karangbolong, way to Petruk Cave; $7^{\circ} 42^{\prime} \mathrm{S}, 109^{\circ} 24^{\prime}$ E; $78 \mathrm{~m}$ a.s.1.; 5 Aug. 2014; A.S. Nurinsiyah, F.L.H. Irsyad, F.J. Sari and E. Nurlaela leg.; D = 8.7 mm, H = $5.2 \mathrm{~mm}$; MZB 19203.

\section{Paratypes}

INDONESIA - Central Java • 6 spec.; Karangbolong, near Petruk Cave, agroforestry; $7^{\circ} 42^{\prime} 17^{\prime \prime} \mathrm{S}$, 109²3'59" E; 78 m a.s.l.; MZB 19273 • 2 spec.; same data as for preceding; ZMH $133409 \bullet 1$ spec.; same data as for preceding; MZB $19204 \cdot 5$ spec.; Karangbolong, near Petruk Cave, agroforestry; $7^{\circ} 42^{\prime} 19^{\prime \prime}$ S, $109^{\circ} 24^{\prime} 02^{\prime \prime}$ E; 78 m a.sl.; MZB 19265 • 4 spec.; same data as for preceding; ZMH 133417 • 4 spec.; Karangbolong, wall and surroundings of Petruk Cave; $7^{\circ} 42^{\prime} 21^{\prime \prime}$ S, $109^{\circ} 24^{\prime} 03^{\prime \prime}$ E; $104 \mathrm{~m}$ a.s.1.; MZB 19266 • 3 spec.; same data as for preceding; MZB 19267 • 5 spec.; Karangbolong, near Petruk Cave, 
agroforestry; $7^{\circ} 42^{\prime} 18^{\prime \prime} \mathrm{S}, 109^{\circ} 24^{\prime} 05^{\prime \prime} \mathrm{E}$; $138 \mathrm{~m}$ a.s.1.; MZB $19274 \bullet 1 \mathrm{spec}$.; same data as for preceding; ZMH 133407 • 23 spec.; Karangbolong, Adiraja Hill, agroforestry; 742'48" S, 109 24'05" E; $168 \mathrm{~m}$ a.s.l.; MZB 19271 • 4 spec.; Karangbolong, Adiraja Hill, teak plantation; $7^{\circ} 42^{\prime} 50^{\prime \prime}$ S, $109^{\circ} 24^{\prime} 05^{\prime \prime}$ E; $166 \mathrm{~m}$ a.s.l.; MZB 19275 • 3 spec.; same data as for preceding; ZMH 133414.

\section{Description}

SHELl (Fig. 50; Tables 3-4). Depressed conical, with 4.75-5.5 hardly convex whorls; protoconch almost smooth; teleoconch with irregular wrinkles, apical side with indistinct incised spiral lines, umbilical side with more distinct spiral lines; scaly processes all over teleoconch; tuberculate around umbilicus; brownish-corneous; body whorl sharply keeled; aperture oval; upper insertion of peristome slightly descending; peristome expanded, reflexed and slightly thickened; umbilicus slightly eccentric, wide, comprising $24-37 \%$ of shell diameter, hardly obscured by columellar edge.

Genitalia (Figs 19, 33; Table 4). Atrium short; penis divided by a narrowing into a long, broad cylindrical distal part and a slightly dilated proximal part, without penis coecum; membranaceous sheath around distal part of penis connected with proximal part of epiphallus by tissue; flagellum moderately long, broadest near its proximal end, where it rapidly tapers, slightly crenated, without node; vas deferens narrow and long; vagina short, distally dilated; oviduct longer, proximally hardly tapering; peduncle of bursa copulatrix subdivided into a broader, cylindrical distal part and a narrower, longer proximal part. Right ommatophoral retractor runs between penis and vagina.

\section{Remarks}

With regard to the presence of scales all over the teleoconch, L. petrukensis sp. nov. resembles L. tholiformis sp. nov. (Fig. 51) and L. madurensis sp. nov. (Fig. 57). It differs from both species in the smaller (D 8.1-9.8 $\mathrm{mm}$ vs 13.0-14.4 $\mathrm{mm}$ in L. tholiformis sp. nov. and 11.4-12.0 $\mathrm{mm}$ in L. madurensis sp. nov.), more depressed shell (D/H 1.49-1.79 vs 1.77-1.88 in L. tholiformis sp. nov. and 1.75-2.11 in L. madurensis sp. nov.) with fewer whorls (4.75-5.5 vs 6-6.25 in L. tholiformis sp. nov. and 5.5-5.75 in L. madurensis sp. nov.), an on average narrower umbilicus (U/D $0.24-0.37$ vs $0.32-0.40$ in $L$. tholiformis sp. nov. and 0.38-0.40 in L. madurensis sp. nov.) and in the penis that is divided by a constriction into a long, broad, cylindrical distal part and a slightly dilated proximal part.

We could only investigate the anatomy of the holotype, from Karangbolong in southern Central Java (MZB 19203). There are several samples from other regions of Central Java, West Java and Banten with similar shells. Thus, it is possible that this species is more widely distributed, but the identity of the samples from other regions must be confirmed anatomically.

\section{Distribution}

Landouria petrukensis sp. nov. is known from Karangbolong in southern Central Java (Fig. 52).

Landouria tholiformis sp. nov. urn:lsid:zoobank.org:act:C083E997-420A-4CAA-8A67-088C02177B83

Figs 7, 20, 34, 51-52; Tables 3-4

\section{Diagnosis}

Landouria tholiformis sp. nov. is characterized by a brownish-corneous, sharply keeled shell with scaly processes all over the shell, with a bulge at the proximal end of the penis forming a short coecum, and a crenated flagellum, which tapers abruptly at its proximal end. 


\section{Etymology}

Landouria tholiformis sp. nov. is named after its dome-shaped shell (Latin: 'tholus' = 'dome', 'forma' $=$ 'shape'; used as an adjective).

\section{Material examined}

\section{Holotype}

INDONESIA - holotype (det. anat.); Gunung Kidul, Tepus, near Poktunggal Beach, agroforestry; $8^{\circ} 08^{\prime} 42^{\prime \prime}$ S, $110^{\circ} 37^{\prime} 52^{\prime \prime}$ E; 137 m a.s.1.; 9 Aug. 2014; A.S. Nurinsiyah, F.L.H. Irsyad, F.J. Sari and E. Nurlaela leg.; $\mathrm{D}=8.7 \mathrm{~mm}, \mathrm{H}=5.2 \mathrm{~mm}$; MZB 17999 .

\section{Paratypes}

INDONESIA - 8 spec.; Yogyakarta, Gunung Kidul, Tepus, Pego Hill, agroforestry; $8^{\circ} 06^{\prime} 10^{\prime \prime}$ S, $110^{\circ} 37^{\prime} 50^{\prime \prime} \mathrm{E} ; 230 \mathrm{~m}$ a.s.1.; MZB 19299.

\section{Description}

SHell (Figs 7, 51; Tables 3-4). Depressed conical, with a dome-shaped spire, with 6-6.25 slightly convex whorls; protoconch almost smooth; teleoconch with fine, irregular wrinkles; apical side with indistinct incised spiral lines, umbilical side with more distinct spiral lines; scaly processes all over shell; tuberculate around umbilicus; brownish-corneous; body whorl sharply keeled; aperture rounded rhombic; upper insertion of peristome descending; peristome expanded, reflexed and thickened; umbilicus slightly eccentric, wide, comprising $32-40 \%$ of shell diameter, hardly obscured by columellar edge.

Genitalia (Figs 20, 34; Table 4). Atrium short; penis long, becoming slightly broader proximally, with a bulge at proximal end forming a short coecum; membranaceous sheath around distal part of penis connected with epiphallus by tissue; penial retractor runs from diaphragm to proximal part of cylindrical epiphallus; flagellum moderately long, broadest in its middle part, crenated, without node, abruptly tapering at its proximal end; vas deferens narrow and long; vagina moderately long; oviduct short, broad, proximally tapering; peduncle of bursa copulatrix subdivided into a broader, almost cylindrical distal part that abruptly passes into a narrower and longer proximal part that ends in an elongate oval bursa beside albumen gland. Right ommatophoral retractor runs between penis and vagina.

\section{Remarks}

Landouria tholiformis sp. nov. clusters in the phylogeny (Fig. 1) with L. abdidalem sp. nov. (Fig. 56). It differs from that species in the more depressed (D/H 1.77-1.88 vs 1.93-2.14 in L. abdidalem sp. nov.), less sharply keeled shell with scaly processes all over the teleoconch (Fig. 7), in the flagellum, which is slightly longer than the short epiphallus, and in having a bulge at the proximal end of the penis (perhaps this is an individual abnormity).

Landouria tholiformis sp. nov. occurs sympatrically with L. sewuensis sp. nov. (Fig. 58), from which it differs in the less sharply keeled shell with a dome-shaped spire, a more distinct granular microsculpture, with scaly processes all over the shell (Fig. 7) and less distinct spiral lines at the umbilical side, the bulge at the proximal end of the penis that forms a short coecum, and a narrow, crenated flagellum. The flagellum of $L$. sewuensis sp. nov. is broader than that of $L$. tholiformis sp. nov. and equipped with two rows of tubercles. There is a globular bulge in the distal part of the vagina in L. sewuensis sp. nov. which is absent in L. tholiformis sp. nov.

\section{Distribution}

Landouria tholiformis sp. nov. is endemic to the Gunung Sewu karst region in Gunung Kidul Regency (Fig. 52). 
Landouria abdidalem sp. nov. urn:lsid:zoobank.org:act:B91C66B0-56B9-40EB-800E-4C321959C0B2

Figs 21, 35, 53, 56; Tables 3-4

\section{Diagnosis}

Landouria abdidalem sp. nov. is characterized by a sharply keeled shell, a long and narrow penis and epiphallus, and a crenated flagellum, which is shorter than the epiphallus and abruptly tapering at its proximal end.

\section{Etymology}

The name of the species is derived from the Javanese expression 'Abdi Dalem' (used as a noun in apposition). Abdi Dalem is a person who devotes himself to serve the Keraton of Yogyakarta (Yogya Palace) and the Sultan.

\section{Material examined}

\section{Holotype}

INDONESIA - holotype (det. anat.); Yogyakarta, Kulonprogo, Jatimulyo, near Kiskendo Cave; 744'53" S, 11007'53" E; 700 m a.s.1.; 25 Aug. 2017; B. Hausdorf leg.; D = 13.4 mm, H = 6.8 mm; MZB 21271.

\section{Paratypes}

INDONESIA - Central Java 1 spec. (det. anat.); Purworejo, Donorejo, near Seplawan Cave, shrubs and limestone rock; $7^{\circ} 45^{\prime} 52^{\prime \prime} \mathrm{S}, 110^{\circ} 05^{\prime} 46^{\prime \prime} \mathrm{E}$; $700 \mathrm{~m}$ a.s.1.; MZB $17733 \cdot 3 \mathrm{spec}$.; same data as for preceding; MZB 17732 • 5 spec.; same data as for preceding; MZB $19314 \bullet 4$ spec.; same data as for preceding; ZMH 133410 • 1 spec.; same data as for preceding; ZMH $133421 \cdot 10$ spec.; Yogyakarta, Kulonprogo, Jatimulyo, near Kiskendo Cave; 744'53" S, 11007'53" E; 700 m a.s.1.; ZMH 140026 • 1 spec. (det. anat.); Yogyakarta, Kulonprogo, Sabrang Kidul, snakefruit plantation; $7^{\circ} 44^{\prime} 38^{\prime \prime}$ S, $110^{\circ} 07^{\prime} 55^{\prime \prime} \mathrm{E} ; 730 \mathrm{~m}$ a.s.1.; MZB 17664 • 7 spec.; same data as for preceding; MZB 19253.

\section{Description}

Shell (Fig. 56; Tables 3-4). Depressed conical, with 5.75-6 hardly convex whorls; protoconch almost smooth; teleoconch with fine, irregular wrinkles; apical side with indistinct incised spiral lines, umbilical side with more distinct spiral lines; scaly processes sparse, mainly at periphery and at umbilical side; tuberculate around umbilicus; brownish-corneous; body whorl sharply keeled; aperture rounded rhombic; upper insertion of peristome descending; peristome expanded, reflexed and thickened; umbilicus hardly eccentric, wide, comprising $33-48 \%$ of shell diameter, hardly obscured by columellar edge.

Genitalia (Figs 21, 35; Table 4). Atrium short; penis long, becoming slightly broader proximally, without penis coecum; membranaceous sheath around distal part of penis connected with the epiphallus by tissue; penial retractor runs from diaphragm to proximal part of cylindrical epiphallus; flagellum shorter than epiphallus, broadest in its middle part, without node, abruptly tapering towards its blunt proximal end, crenated; vas deferens narrow and long; vagina long; oviduct shorter, broad, proximally tapering; peduncle of bursa copulatrix subdivided into a broader, spindle-shaped distal part and a narrower, longer proximal part, ending in an elongate oval bursa beside albumen gland. Right ommatophoral retractor runs between penis and vagina.

\section{Remarks}

With regard to the shell, L. abdidalem sp. nov. resembles L. tholiformis sp. nov. (Fig. 51), with which it clusters in the molecular phylogeny (Fig. 1). For differences, see remarks under that species. The range 


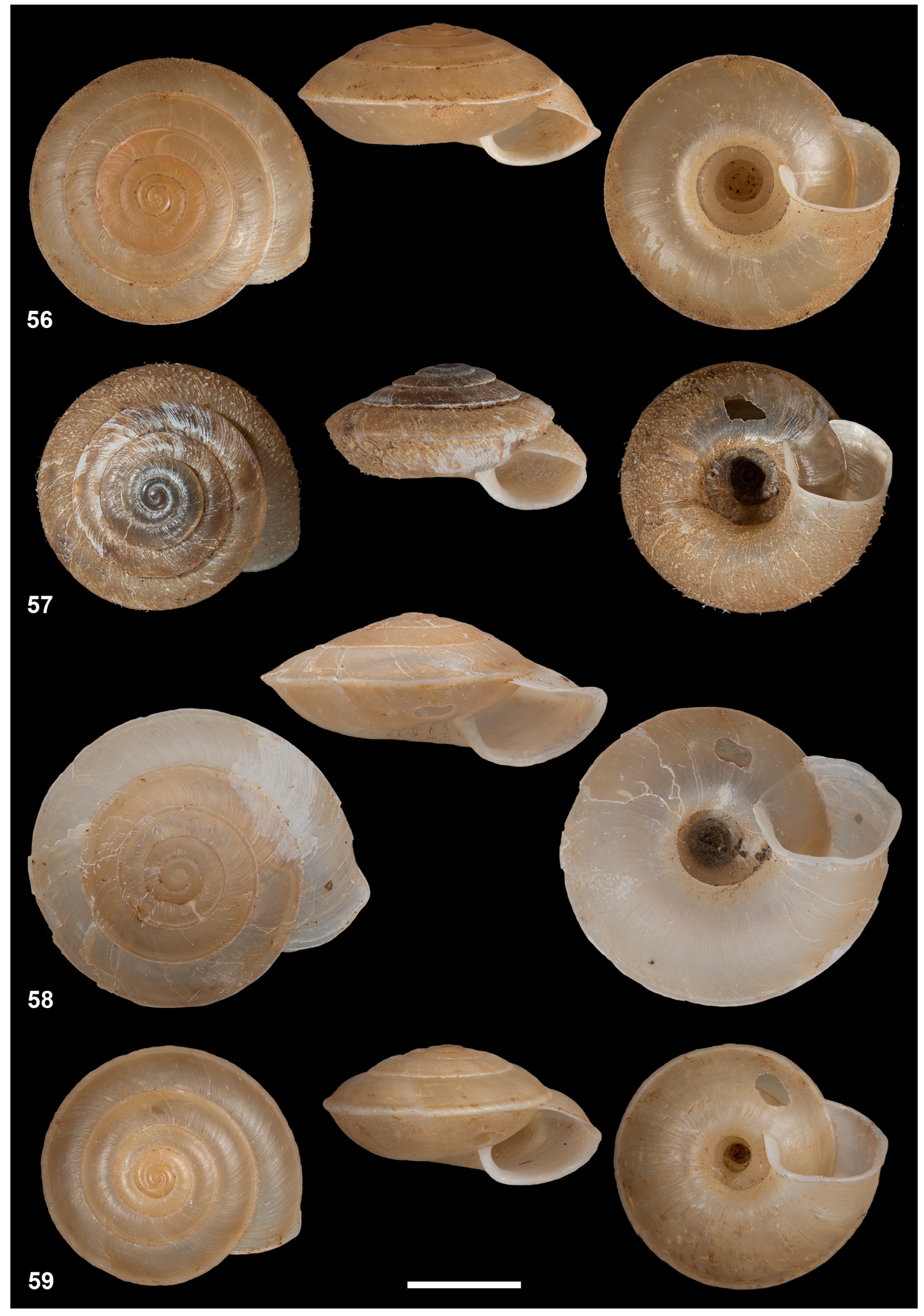


of $L$. abdidalem sp. nov. overlaps with that of $L$. zonifera sp. nov. For differences, see remarks under that species. Furthermore, the range of $L$. abdidalem sp. nov. overlaps with that of $L$. menorehensis sp. nov. (Fig. 88) from which it can easily be distinguished by the sharply keeled, depressed conical, brownishcorneous shell.

\section{Distribution}

Landouria abdidalem sp. nov. is endemic to the Menoreh Hills (Fig. 53).

Landouria madurensis sp. nov. urn:1sid:zoobank.org:act:024A8B78-91C2-49CA-8721-C0B8CCC46982

Figs 8, 22, 36, 53, 57; Tables 3-4

Helix squamulosa Martens, 1867: 266, pl. 13, fig. 10 ("Insel Madura (an der Nordostseite von Java), bei Sumanap") (objective junior synonym of Helix squamulosa Mousson, 1856). Syn. nov.

Helix squamulosa - Pfeiffer 1871 in 1870-1876: pl. 117, figs 13-15; 1872 in 1870-1876: 41.

Landouria squamulosa - van Benthem Jutting 1941: 311.

\section{Diagnosis}

Landouria madurensis sp. nov. is characterized by a sharply keeled shell with longish scales all over the teleoconch, a wide umbilicus, a long penis, which is divided into a longer, broader distal and a shorter, narrower proximal part, and a long flagellum, which gradually tapers towards its proximal end.

\section{Etymology}

Landouria madurensis sp. nov. is named after its occurrence on Madura Island.

\section{Material examined}

\section{Holotype}

INDONESIA - holotype (det. anat.); East Java, Madura Island, Sampang, Nepa Monkey lowland rainforest; $6^{\circ} 54^{\prime} 01^{\prime \prime} \mathrm{S}, 113^{\circ} 12^{\prime} 08^{\prime \prime} \mathrm{E} ; 17 \mathrm{~m}$ a.s.1.; 14 May 2014; A.S. Nurinsiyah and T. Setiadi leg.; $\mathrm{D}=11.4 \mathrm{~mm}, \mathrm{H}=6.5 \mathrm{~mm}$; MZB 21432 .

\section{Paratypes}

INDONESIA • 1 spec.; same data as for holotype; MZB 19209 • 4 spec.; same data as for holotype; MZB $19210 \cdot 1 \mathrm{spec} . ;$ same data as for holotype; ZMH $133408 \cdot 4$ spec.; same data as for holotype; ZMH $133437 \cdot 2$ spec. (also syntypes of H. squamulosa); Sumenep (see Martens 1867: 267); $7^{\circ} 01^{\prime}$ S, $113^{\circ} 52^{\prime} \mathrm{E} ; \mathrm{ZMB} 7155$.

\section{Description}

Shell (Figs 8, 57; Tables 3-4). Depressed conical, with 5.5-5.75 hardly convex whorls; protoconch almost smooth; teleoconch with irregular wrinkles; without distinct incised spiral lines; longish scales

Figs 56-59 (opposite page). Shells of species of Landouria from Java. 56. L. abdidalem sp. nov., Yogyakarta, Kulonprogo, Sabrang Kidul (holotype, MZB 17664). 57. L. madurensis sp. nov., East Java, Madura Island, Sampang, Nepa Monkey lowland rainforest (holotype MZB 21432). 58. L. sewuensis sp. nov., Yogyakarta, Gunung Kidul, Tepus, Mbajo Hill (holotype, MZB 19255). 59. L. sukoliloensis sp. nov., Central Java, Kayen, near Watu Payung (holotype, MZB 19219). Scale bar: 5 mm. 
all over teleoconch; tuberculate around umbilicus; brownish-corneous; body whorl sharply keeled; aperture rounded rhombic; upper insertion of peristome descending; peristome expanded, reflexed and thickened; umbilicus slightly eccentric, very wide, comprising $38-40 \%$ of shell diameter, hardly obscured by columellar edge.

Genitalia (Figs 22, 36; Table 4). Atrium short; penis long, divided into a longer, broader distal and a shorter, narrower proximal part, without penis coecum; membranaceous sheath around distal part of penis connected with proximal part of epiphallus by tissue; penial retractor runs from diaphragm to middle part of cylindrical epiphallus; flagellum long, broadest in its middle part, gradually tapering towards its proximal end, slightly crenated, without node; vas deferens narrow and long; vagina cylindrical, long; oviduct shorter, proximally hardly tapering; peduncle of bursa copulatrix subdivided into a broader, cylindrical distal part and a narrower, longer proximal part. Right ommatophoral retractor runs between penis and vagina.

\section{Remarks}

Landouria madurensis sp. nov. differs from the neighbouring Landouria intumescens in the presence of longish scales all over the teleoconch (Fig. 8), a wider umbilicus (U/D $0.38-0.40$ vs $0.22-0.34$ in L. intumescens) and a relatively long vagina (P:V 2.1 vs 2.4-3.9 in L. intumescens).

A second, more conical species of Landouria without longish scales and with a narrower umbilicus, of which only juveniles were found, lives sympatrically with Landouria madurensis sp. nov. in Nepa Monkey lowland rainforest on Madura Island.

\section{Distribution}

Landouria madurensis sp. nov. is endemic to Madura Island (Fig. 53).

Landouria sewuensis sp. nov. urn:1sid:zoobank.org:act:2E324442-51A9-4897-9635-22666EF0E037

Figs 23, 37, 53, 58; Tables 3-4

Landouria cf. rotatoria - Köhler et al. in press: 6 (in part). (not Pfeiffer, 1842)

\section{Diagnosis}

Landouria sewuensis sp. nov. is characterized by a brownish-corneous, sharply keeled shell with a wide umbilicus, a penis that is divided into a long, broad distal and a short, narrow proximal part with a globular bulge at its proximal end, a broad flagellum with two rows of broad tubercles and a globular bulge in the distal part of the vagina.

\section{Etymology}

Landouria sewuensis sp. nov. is named after its occurrence in the Gunung Sewu karst region.

\section{Material examined}

\section{Holotype}

INDONESIA • holotype (det. anat.); Gunung Kidul, Tepus, Mbajo Hill, natural forest; $8^{\circ} 08^{\prime} 33^{\prime \prime}$ S, $110^{\circ} 40^{\prime} 59^{\prime \prime}$ E; 249 m a.s.1.; 8 Aug. 2014; A.S. Nurinsiyah, F.L.H. Irsyad, F.J. Sari and E. Nurlaela leg.; $\mathrm{D}=15.1 \mathrm{~mm}, \mathrm{H}=7.1 \mathrm{~mm} ; \mathrm{MZB} 19255$. 


\section{Paratypes}

INDONESIA - Central Java • many spec.; Yogyakarta, Gunung Kidul, Kemadang: Pantai Baron; $8^{\circ} 07^{\prime} 43^{\prime \prime}$ S, 110 $32^{\prime} 54^{\prime \prime}$ E; 20 m a.s.1.; ZMH $140024 \bullet 2$ spec. (det. anat.); Gunung Kidul, Tepus, Cemara Udang Hill, secondary forest; 808'55" S, 110³6'37" E; $39 \mathrm{~m}$ a.s.1.; MZB $19261 \bullet 1 \mathrm{spec}$.; same data as for preceding; ZMH 133418 - many spec.; Gunung Kidul, Tepus, rock above Poktunggal Beach; $8^{\circ} 09^{\prime 2} 23^{\prime \prime}$ S, $110^{\circ} 37^{\prime 22}$ " E; 10 m a.s.1.; ZMH 140025 • 1 spec. (det. anat.); Gunung Kidul, Tepus, Pego Hill, agroforestry; $8^{\circ} 06^{\prime} 10^{\prime \prime} \mathrm{S}, 110^{\circ} 37^{\prime} 50^{\prime \prime} \mathrm{E} ; 230 \mathrm{~m}$ a.s.l.; MZB $19259 \bullet 1$ spec. (det. anat.); Gunung Kidul, Tepus, Pego Hill, agroforestry; 806'10" S, 110³7'51" E; 244 m a.s.l.; MZB 19258 • 1 spec.; Gunung Kidul, Tepus, near Poktunggal Beach, agroforestry; 808'42" S, $110^{\circ} 37^{\prime} 52^{\prime \prime}$ E; $137 \mathrm{~m}$ a.s.l.; MZB $21269 \bullet 3$ spec. (det. anat.); same data as for preceding; MZB $19260 \bullet 3$ spec.; same data as for preceding; ZMH 79891 - 1 spec. (det. anat.); Gunung Kidul, Tepus, Mbajo Hill, natural forest; $8^{\circ} 08^{\prime} 32^{\prime \prime}$ S, $110^{\circ} 40^{\prime} 58^{\prime \prime}$ E; $281 \mathrm{~m}$ a.s.1.; MZB 19257 • 1 spec.; same data as for preceding; ZMH $133419 \bullet 1$ spec. (det. anat.); Gunung Kidul, Tepus, Mbajo Hill, natural forest; 808'33" S, 110 40'59" E; 258 m a.s.l.; MZB 19256.

\section{Description}

SHELl (Fig. 58; Tables 3-4). Depressed conical, with 5.25-6 hardly convex whorls; protoconch almost smooth; teleoconch with fine, irregular wrinkles; apical side with indistinct incised spiral lines, umbilical side with more distinct spiral lines; scaly processes, if present, at periphery; tuberculate around umbilicus; brownish-corneous; body whorl sharply keeled; aperture rounded rhombic; upper insertion of peristome slightly descending; peristome expanded, reflexed and slightly thickened; umbilicus slightly eccentric, wide, comprising $30-40 \%$ of shell diameter, hardly obscured by columellar edge.

GenitaLia (Figs 23, 37; Table 4). Atrium short; penis long, distal part broad, shorter proximal part narrow, with a globular swelling at proximal end; without penis coecum; membranaceous sheath around distal part of penis connected with proximal part of epiphallus by tissue; penial retractor runs from diaphragm to middle of cylindrical epiphallus; flagellum moderately long, broad, with two rows of broad tubercles at margin, tapering towards its proximal end; without node; vas deferens narrow and long; vagina long, distally globularly dilated, constriction towards its middle, becoming broader again towards its proximal end; oviduct short, broad, proximally tapering; peduncle of bursa copulatrix subdivided into a broader, cylindrical distal part and a narrower, longer proximal part, ending in an elongate oval bursa beside albumen gland. Right ommatophoral retractor runs between penis and vagina.

\section{Remarks}

The shell of $L$. sewuensis sp. nov. cannot be reliably distinguished from those of $L$. sukoliloensis sp. nov. (Fig. 59) or L. intumescens (Figs 72-74). Landouria sewuensis sp. nov. differs from these species in the division of the penis into a long, broad distal and a short, narrow proximal part with a globular bulge at its proximal end, the broad flagellum with two rows of broad tubercles and the globular bulge in the distal part of the vagina.

Landouria sewuensis sp. nov. occurs sympatrically with L. tholiformis sp. nov. (Fig. 51). For differences, see remarks under that species.

A sequence of $L$. sewuensis sp. nov. was included in the phylogenetic analysis of Köhler et al. (in press: fig. 2; ZMH 79891; erroneously labelled "Sumatra") under the name $L$. cf. rotatoria (not Pfeiffer, 1842).

\section{Distribution}

Landouria sewuensis sp. nov. is endemic to the Gunung Sewu karst region in Gunung Kidul Regency (Fig. 53). 
Landouria sukoliloensis sp. nov. urn:1sid:zoobank.org:act:74853E9E-ECA0-4182-8CB2-FD09A4CD903C

Figs 24, 38, 53, 59; Tables 3-4

Landouria winteriana - Nurinsiyah 2015: 32. (not Pfeiffer, 1842)

\section{Diagnosis}

Landouria sukoliloensis sp. nov. is characterized by a brownish-corneous, sharply keeled shell, a penis that is dilated at its proximal end and a flagellum that is not crenated, but abruptly tapering at its proximal end.

\section{Etymology}

Landouria sukoliloensis sp. nov. is named after its occurrence in the Sukolilo Karst.

\section{Material examined}

Holotype

INDONESIA - holotype (det. anat.); Central Java, Pati, Kayen, near Watu Payung; 6 $55^{\prime} 59^{\prime \prime}$ S, $110^{\circ} 55^{\prime} 34^{\prime \prime}$ E; 110 m a.s.1.; 16 Aug. 2014; A.S. Nurinsiyah, F.L.H. Irsyad, F.J. Sari and E. Nurlaela leg.; $\mathrm{D}=11.7 \mathrm{~mm}, \mathrm{H}=6.1 \mathrm{~mm} ; \mathrm{MZB} 19219$.

\section{Paratypes}

INDONESIA - Central Java 7 spec. (det. anat.); Grobogan, Taruman Village, near Sendang Sedayu, agroforestry; $7^{\circ} 00^{\prime} 52^{\prime \prime} \mathrm{S}, 110^{\circ} 51^{\prime} 29^{\prime \prime} \mathrm{E}$; $20 \mathrm{~m}$ a.s.1.; MZB $19221 \bullet 1 \mathrm{spec}$.; same data as for preceding; ZMH 133432 - 5 spec.; Grobogan, Taruman Village, near Sendang Sedayu, agroforestry; $7^{\circ} 00^{\prime} 52^{\prime \prime}$ S, $110^{\circ} 51^{\prime 2} 29^{\prime \prime}$ E; 23 m a.s.1.; MZB 19216 • 2 spec.; same data as for preceding; ZMH 133435 • 13 spec.; same data as for holotype; MZB 19218 • 9 spec.; same data as for holotype; ZMH $133433 \bullet 10$ spec.; Pati, Kayen, near Watu Payung; 6 ${ }^{\circ} 56^{\prime} 00^{\prime \prime}$ S, $110^{\circ} 55^{\prime} 34^{\prime \prime}$ E; 115 m a.s.1.; MZB $19225 \bullet 4$ spec.; same data as for preceding; ZMH $133429 \cdot 21$ spec. (det. anat.); Pati, Sukolilo, Kedumulyo, Wareh Cave, agroforestry; 6 $6^{\circ} 55^{\prime} 41^{\prime \prime} \mathrm{S}, 110^{\circ} 57^{\prime} 37^{\prime \prime} \mathrm{E} ; 73 \mathrm{~m}$ a.s.1.; MZB $192229 \bullet 15$ spec.; same data as for preceding; ZMH 133431 • 7 spec.; Pati, Sukolilo, Kedumulyo, Wareh Cave, agroforestry; 6 ${ }^{\circ} 55^{\prime} 41^{\prime \prime}$ S, $110^{\circ} 57^{\prime} 38^{\prime \prime}$ E; $53 \mathrm{~m}$ a.s.1.; MZB 19223 • 4 spec.; same data as for preceding; ZMH 133430.

\section{Other material}

INDONESIA - Central Java 3 spec.; Grobogan, Sedayu, surroundings of Macan Cave, agroforestry and shrubs; $6^{\circ} 58^{\prime} 30^{\prime \prime}$ S, $110^{\circ} 57^{\prime} 03^{\prime \prime}$ E; 363 m a.s.1.; MZB 19252 • 3 spec.; Pati, Kayen, Brati, Nggoboyo Spring, agroforestry; $6^{\circ} 54^{\prime} 58^{\prime \prime} \mathrm{S}, 111^{\circ} 01^{\prime} 54^{\prime \prime}$ E; 89 m a.s.l.; MZB $19232 \cdot 3$ spec.; Pati, Tambakromo,

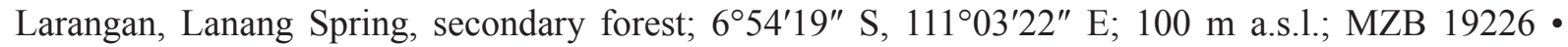
2 spec.; Pati, Tambakromo, Larangan, Lowo Cave, secondary forest; 6 $54^{\prime} 19^{\prime \prime}$ S, $111^{\circ} 03^{\prime} 24^{\prime \prime}$ E; 112 m a.s.l.; MZB 19230 - 1 spec.; Pati, Tambakromo, Kedunglawah, Ngencek Spring, agroforestry; 6 $54^{\prime} 05^{\prime \prime}$ S, $111^{\circ} 03^{\prime} 57^{\prime \prime}$ E; 110 m a.s.1.; MZB 19262.

\section{Description}

SHELl (Fig. 59; Tables 3-4). Depressed conical, with 5-5.75 hardly convex whorls; protoconch almost smooth; teleoconch with fine, irregular wrinkles, indistinct incised spiral lines, umbilical side with more distinct spiral lines; scaly processes sparse, mainly at periphery; tuberculate around umbilicus; brownish-corneous; body whorl sharply keeled; aperture rounded rhombic; upper insertion of peristome slightly descending; peristome expanded, slightly reflexed and thickened; umbilicus slightly eccentric, wide, comprising $22-41 \%$ of shell diameter, hardly obscured by columellar edge. 
Genitalia (Figs 24, 38; Table 4). Atrium short; penis long, cylindrical, dilated at its proximal end, without penis coecum; membranaceous sheath around distal part of penis connected with proximal part of epiphallus by tissue; penial retractor runs from diaphragm to middle part of almost cylindrical epiphallus; flagellum moderately long, broadest in its middle part, abruptly tapering at its proximal end, not crenated, without node; vas deferens narrow and long; vagina long, often constricted near atrium; oviduct short, broad, proximally tapering; peduncle of bursa copulatrix subdivided into a broader distal part and a narrower, longer proximal part, ending in an elongate oval bursa beside albumen gland. Right ommatophoral retractor runs between penis and vagina.

\section{Remarks}

Landouria sukoliloensis sp. nov. cannot be distinguished from L. sewuensis sp. nov. and L. intumescens by shell characters. It differs from these species in the penis that is dilated at its proximal end and in the flagellum, which is not crenulated but abruptly tapers at its proximal end.

Landouria sukoliloensis sp. nov. differs from L. tonywhitteni sp. nov. (Fig. 60), with which it occurs sympatrically, in the smaller (D 10.4-12.3 $\mathrm{mm}$ vs $14.3-17.6 \mathrm{~mm}$ in L. tonywhitteni sp. nov.), higher shell (D/H 1.60-1.93 vs 1.93-2.34 in L. tonywhitteni sp. nov.), the dilatation at the proximal end of the penis and the lack of a globular node at the flagellum.

\section{Distribution}

Landouria sukoliloensis sp. nov. is endemic to Sukolilo Karst (Fig. 53).

Landouria tonywhitteni sp. nov. urn:lsid:zoobank.org:act:59260748-3438-411F-82B4-14A7847AFA58

Figs 39, 52, 60, 63; Tables 3-4

\section{Diagnosis}

Landouria tonywhitteni sp. nov. is characterized by having a strongly depressed conical, brownishcorneous, sharply keeled shell and a penis that does not become distinctly broader towards is proximal end, the lack of a coecum at the proximal end of the penis and the presence of a node at the base of the flagellum.

\section{Etymology}

Landouria tonywhitteni sp. nov. is named in honour of the late British naturalist Tony Whitten (19532017), who dedicated his life to the conservation of karst habitats and especially the snails that inhabit these habitats.

\section{Material examined}

\section{Holotype}

INDONESIA - holotype (det. anat.); Central Java, Grobogan, Taruman, near Sendang Sedayu; $7^{\circ} 00^{\prime} 52^{\prime \prime}$ S, 110 51'29" E; 20 m a.s.l.; 16 Aug. 2014; A.S. Nurinsiyah, F.L.H. Irsyad, F.J. Sari and E. Nurlaela leg.; measurements: D = 17.6 mm, H = 7.6 mm; MZB 19214 .

\section{Paratypes}

INDONESIA - Central Java • 9 spec.; same data as for holotype; MZB $19217 \cdot 5$ spec.; same data as for holotype; ZMH $133434 \cdot 9$ spec. (det. anat.); Grobogan, Taruman, near Sendang Sedayu; $7^{\circ} 00^{\prime} 52^{\prime \prime}$ S, $110^{\circ} 51^{\prime} 29^{\prime \prime}$ E; 23 m a.s.1.; MZB 19215 • 1 spec.; same data as for preceding; MZB $21268 \bullet 5$ spec.; same data as for preceding; ZMH 133436. 


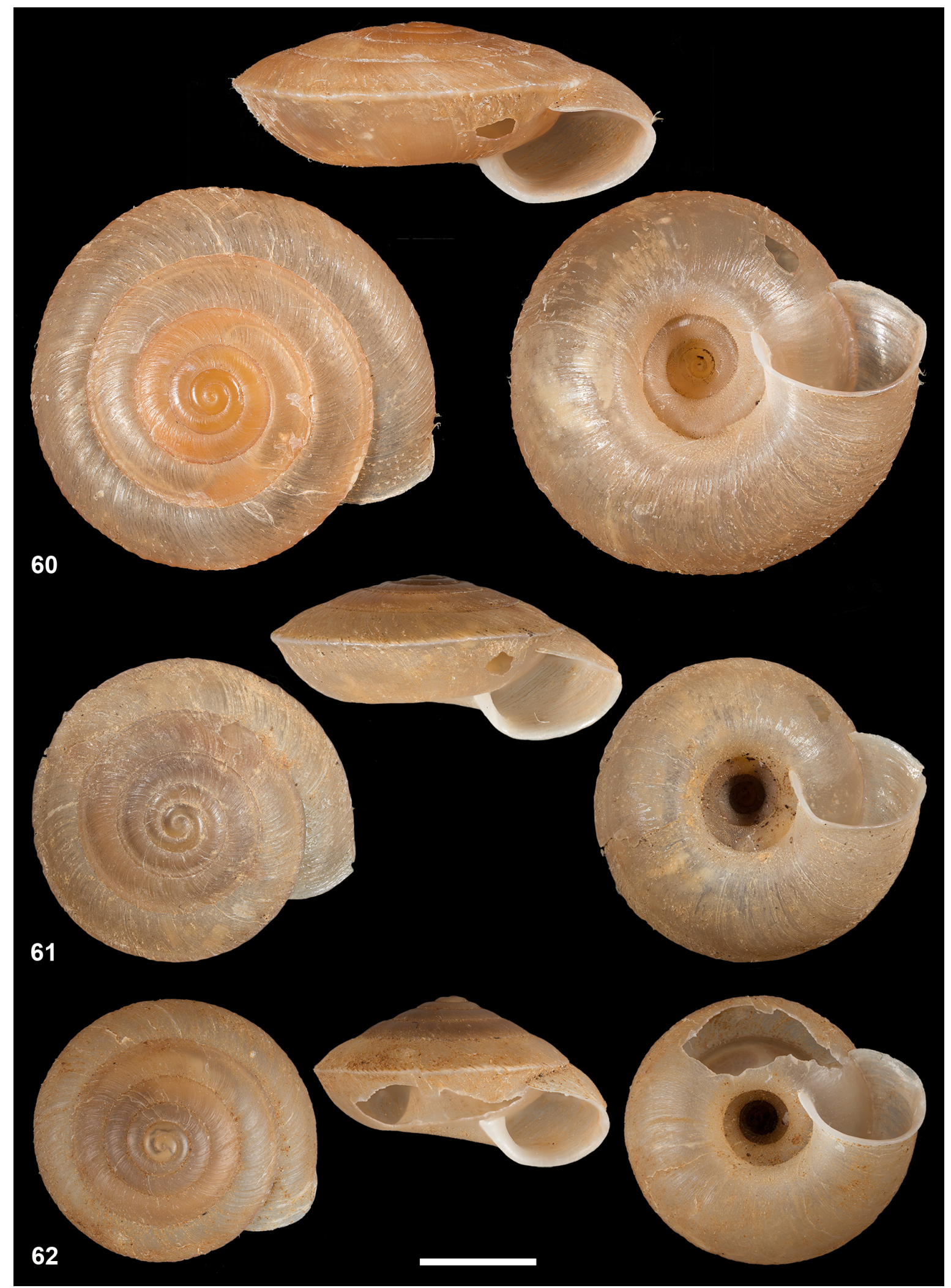

Figs 60-62. Shells of species of Landouria from Java. 60. L. tonywhitteni sp. nov., Central Java, Taruman Village, near Sendang Sedayu (holotype, MZB 19214). 61. L. nodifera sp. nov., Central Java, Larangan Village, Lanang Spring (holotype, MZB 19227). 62. L. pacitanensis sp. nov., East Java, Pacitan, Srau Beach (holotype, MZB 21191). Scale bar: $5 \mathrm{~mm}$. 


\section{Description}

SHELL (Fig. 60; Tables 3-4). Depressed conical, with 5.75-6 hardly convex whorls; protoconch almost smooth; teleoconch with fine, irregular wrinkles; apical side with indistinct spiral lines, umbilical side with indistinct incised spiral lines; with radial scaly processes; tuberculate around umbilicus; brownishcorneous; body whorl sharply keeled; aperture rhombic; upper insertion of peristome slightly descending; peristome expanded, reflexed and slightly thickened; umbilicus slightly eccentric, wide, comprising $32-41 \%$ of shell diameter, hardly obscured by columellar edge.

Genitalia (Figs 39, 63; Table 4). Atrium short; penis long, consisting of a long, cylindrical distal part covered by an additional sheath, which is connected by muscular tissue to proximal part of epiphallus and a dilated proximal part containing a short penial papilla; without penis coecum; penial retractor runs from diaphragm to distal part of long, narrow epiphallus; flagellum short, broadest at its base, where it is equipped with a globular node, tapering towards its proximal end, not distinctly crenated; vas deferens narrow and long; vagina long; peduncle of bursa copulatrix subdivided into a slightly broader, cylindrical distal part and a narrower, longer proximal part, ending in an elongate oval bursa beside albumen gland. Right ommatophoral retractor runs between penis and vagina.

\section{Remarks}

Landouria nodifera sp. nov., L. tonywhitteni sp. nov., L. pacitanensis sp. nov., L. sewuensis sp. nov. and $L$. intumescens share strongly depressed, brownish-corneous, sharply keeled shells. These species cannot be reliably distinguished by shell characters. Landouria tonywhitteni sp. nov. shares a node at the base of the flagellum with $L$. nodifera sp. nov., from which it differs in the lack of a coecum at the proximal end of the penis and in having a penis that does not become broader towards its proximal end.

\section{Distribution}

Landouria tonywhitteni sp. nov. is endemic to Sukolilo Karst (Fig. 52), where it occurs sympatrically with L. sukoliloensis sp. nov.

Landouria nodifera sp. nov.

urn:1sid:zoobank.org:act:36465425-2C50-4721-9B73-3719E2D4A78A

Figs 40, 54, 61, 64; Tables 3-4

Landouria rotatoria - Nurinsiyah 2015: 32 (in part). (not Pfeiffer, 1842)

\section{Diagnosis}

Landouria nodifera sp. nov. is characterized by a strongly depressed conical, brownish-corneous, sharply keeled shell and a penis that becomes broader towards is proximal end, the presence of a coecum at the proximal end of the penis and a node at the base of the flagellum.

\section{Etymology}

Landouria nodifera sp. nov. is named after the node at its flagellum (Latin: 'nodus' = 'node', 'ferre' = 'to carry'; used as an adjective).

\section{Material examined}

Holotype

INDONESIA • holotype (det. anat.); Central Java, Pati, Larangan Village, Lanang Spring; 6 ${ }^{\circ} 54^{\prime} 19^{\prime \prime}$ S, $111^{\circ} 03^{\prime} 22^{\prime \prime}$ E; 100 m a.s.l.; 15 Aug. 2014; A.S. Nurinsiyah, F.L.H. Irsyad, F.J. Sari and E. Nurlaela leg.; measurements: $\mathrm{D}=15.0 \mathrm{~mm}, \mathrm{H}=7.0 \mathrm{~mm}$; MZB 19227 . 
Paratypes

INDONESIA - Central Java 11 spec.; same data as for holotype; MZB $19233 \bullet 6$ spec.; same data as for holotype; MZB $19228 \cdot 5 \mathrm{spec}$.; same data as for holotype; ZMH $133428 \bullet 9 \mathrm{spec}$. (det. anat.); Pati,

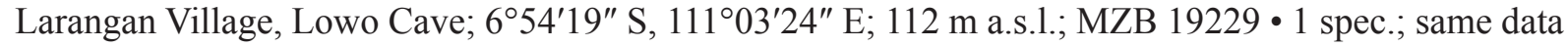
as for preceding; MZB 21266.

\section{Description}

SHELl (Fig. 61; Tables 3-4). Depressed conical, with 5-5.75 hardly convex whorls; protoconch almost smooth; teleoconch with irregular wrinkles; apical side with indistinct incised spiral lines, umbilical side with more distinct spiral lines; scaly processes sparse, mainly at periphery; tuberculate around umbilicus; brownish-corneous; body whorl sharply keeled; aperture rhombic; upper insertion of peristome slightly descending; peristome expanded, reflexed and slightly thickened; umbilicus slightly eccentric, wide, comprising $26-38 \%$ of shell diameter, hardly obscured by columellar edge.

Genitalia (Figs 40, 64; Table 4). Atrium short; penis long, consisting of a cylindrical distal part covered by an additional sheath, connected by muscular tissue to proximal part of epiphallus, and a dilated proximal part containing a short penial papilla; inside proximal part of penis with longitudinal pilasters, which pass into a more complex sculpture in distal part of penis; at proximal end of penis with a blunt penis coecum; penial retractor runs from diaphragm to distal part of epiphallus, which tapers proximally; flagellum moderately long, broadest in its middle part, where it is equipped with a node, tapering towards its proximal end; vas deferens narrow and long; vagina long; peduncle of bursa copulatrix subdivided into a broader, cylindrical distal part and a narrower, longer proximal part, ending in an elongate oval bursa beside albumen gland. Right ommatophoral retractor runs between penis and vagina.

\section{Remarks}

Landouria nodifera sp. nov., L. tonywhitteni sp. nov., L. pacitanensis sp. nov., L. sukoliloensis sp. nov., L. sewuensis sp. nov. and L. intumescens share strongly depressed, brownish-corneous, sharply keeled shells. These species cannot be reliably distinguished by shell characters. Landouria nodifera sp. nov. shares having a coecum at the proximal end of the penis with L. pacitanensis sp. nov., from which it differs in the lack of the narrow constriction subdividing the penis of L. pacitanensis sp. nov. and the presence of a node at the base of the flagellum. Landouria nodifera sp. nov. shares having a node at the base of the flagellum with L. tonywhitteni sp. nov., from which it differs in the presence of the coecum at the proximal end of the penis and the proximally broader penis.

\section{Distribution}

Landouria nodifera sp. nov. is endemic to Sukolilo Karst (Fig. 54).

Landouria pacitanensis sp. nov. urn:lsid:zoobank.org:act:E0076893-CF05-4D23-A1E0-402A2DE7F0EF

Figs 9, 41, 53, 62, 65; Tables 3-4

\section{Diagnosis}

Landouria pacitanensis sp. nov. is characterized by a strongly depressed conical, brownish-corneous, sharply keeled shell and a penis with a distinct constriction between its distal and its proximal part, and the presence of a coecum at the proximal end of the penis.

\section{Etymology}

Landouria pacitanensis sp. nov. is named after its occurrence in the Pacitan Regency. 


\section{Material examined}

\section{Holotype}

INDONESIA - holotype (det. anat.); East Java, Pacitan, Srau Beach; 8 $15^{\prime} 01^{\prime \prime}$ S, 110 59'46" E; $60 \mathrm{~m}$ a.s.1.; 10 Aug. 2014; A.S. Nurinsiyah, F.L.H. Irsyad, F.J. Sari and E. Nurlaela leg.; measurements: $\mathrm{D}=12.7 \mathrm{~mm}, \mathrm{H}=7.1 \mathrm{~mm}$; MZB 21191 .
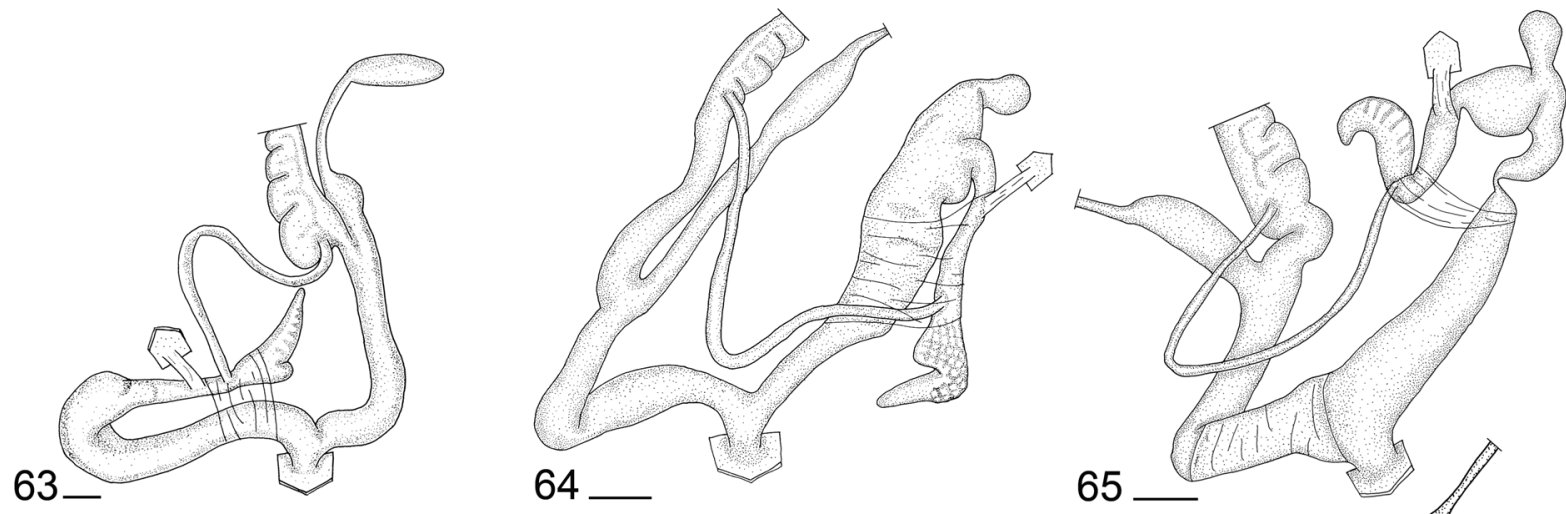

\section{3}
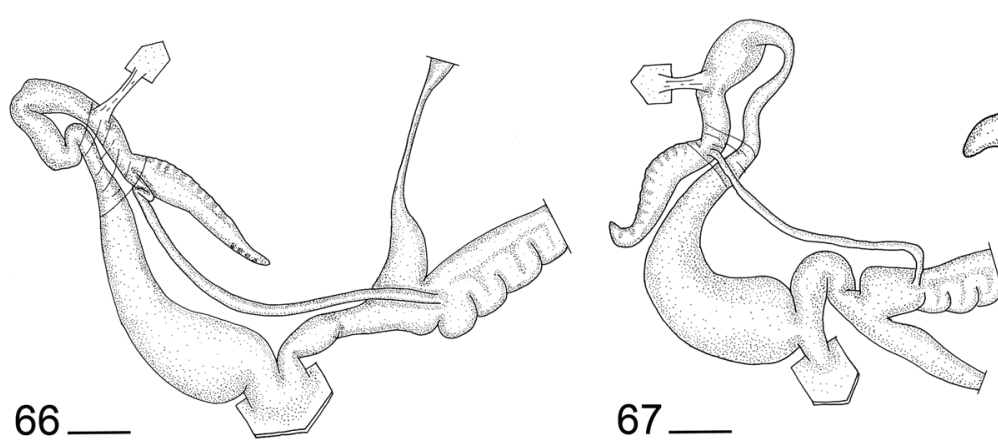

67
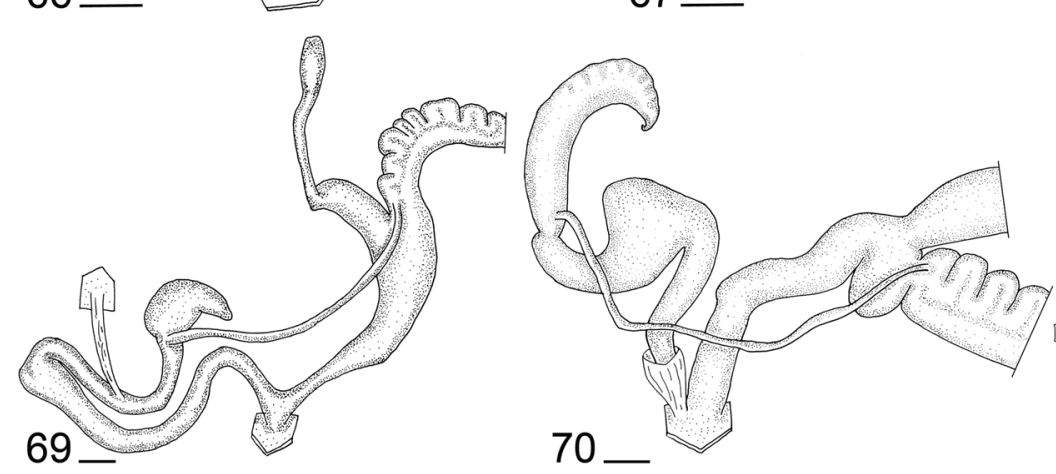

68
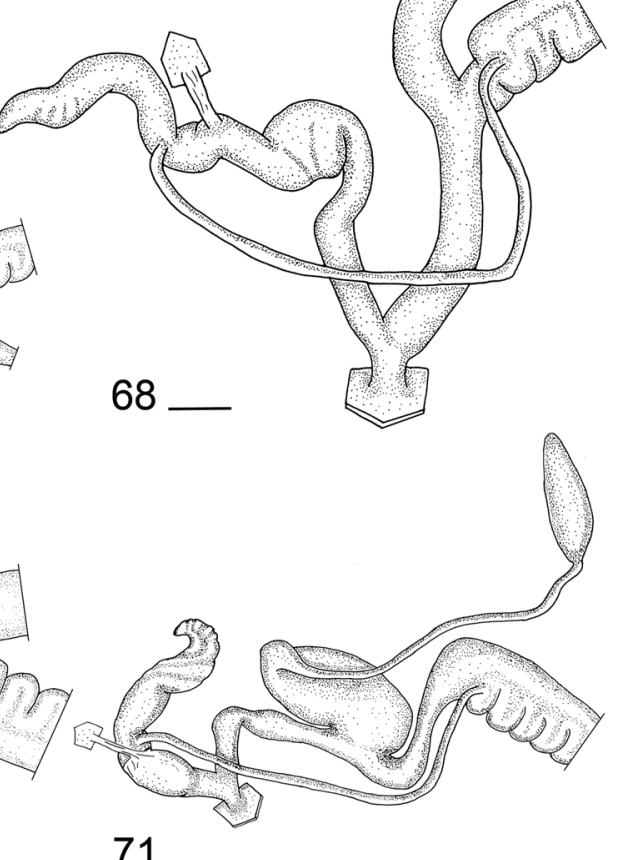

71

Figs 63-71. Genitalia of species of Landouria from Java. 63. L. tonywhitteni sp. nov., Central Java, Taruman Village, near Sendang Sedayu (holotype, MZB 19214). 64. L. nodifera sp. nov., Central Java, Larangan Village, Lanang Spring (holotype, MZB 19227). 65. L. pacitanensis sp. nov., East Java, Pacitan, near Srau Beach (MZB 19231). 66. L. intumescens (Martens, 1867), East Java, Guwoterus (MZB 19207). 67. L. smimensis (Mousson, 1848), East Java, Tosari (ZMA 390881). 68. L. zonifera sp. nov., Yogyakarta, Kulonprogo, Sibolong (holotype, MZB 17727). 69. L. ciliocincta (Möllendorff, 1897), East Java, Malang, Kondangmerak (MZB 19211). 70. L. pakidulan sp. nov., Central Java, Nusa Kambangan Island, nature monument Gligir (holotype, ZMA 417011a). 71. L. menorehensis sp. nov., Yogyakarta, Kulonprogo, Sibolong (holotype, MZB 19201). Scale bars: $1 \mathrm{~mm}$. 


\section{Paratypes}

INDONESIA - East Java • 7 spec.; Pacitan, Srau Beach; 8¹4'58" S, 110 59'42" E; 78 m a.s.1.; MZB $19304 \cdot 4$ spec.; same data as for preceding; ZMH $148511 \bullet 6$ spec.; same data as for holotype; MZB 19234 • 11 spec.; same data as for holotype; MZB 21270 • 4 spec.; same data as for holotype; ZMH $148510 \bullet 6$ spec.; same data as for holotype; ZMH 148513.

\section{Other material}

INDONESIA - East Java • 1 spec. (det.anat.); Pacitan, near Srau Beach; 8 ${ }^{\circ} 13^{\prime} 55^{\prime \prime}$ S, $111^{\circ} 00^{\prime} 54^{\prime \prime}$ E; $217 \mathrm{~m}$ a.s.1.; MZB $21267 \cdot 11 \mathrm{spec}$.; same data as for preceding; MZB $19231 \cdot 5 \mathrm{spec}$.; same data as for preceding; ZMH 148509 • 4 spec.; same data as for preceding; ZMH 148512 • 7 spec.; Pacitan, near Srau Beach; $8^{\circ} 13^{\prime} 54^{\prime \prime}$ S, $111^{\circ} 00^{\prime} 55^{\prime \prime}$ E; $223 \mathrm{~m}$ a.s.1.; MZB 21192 • 5 spec.; same data as for preceding; ZMH 148507.

\section{Description}

SHell (Figs 9, 62; Tables 3-4). Depressed conical, with 5.25-6 slightly convex whorls; protoconch almost smooth; teleoconch with fine, irregular wrinkles; apical side with indistinct incised spiral lines, umbilical side with more distinct spiral lines; scaly processes sparse, mainly at periphery; tuberculate around umbilicus; brownish-corneous; body whorl sharply keeled; aperture almost circular; upper insertion of peristome descending; peristome expanded, reflexed and slightly thickened; umbilicus hardly eccentric, wide, comprising $28-34 \%$ of shell diameter, hardly obscured by columellar edge.

Genitalia (Figs 41, 65; Table 4). Atrium short; penis long, consisting of a long, cylindrical and very muscular distal part covered by an additional sheath, which is connected by muscular tissue to proximal part of epiphallus, and a dilated proximal part; distal and proximal parts of penis separated by a narrow constriction; penial papilla indistinct; inside proximal part of penis with longitudinal pilasters, which pass into a more complex sculpture in distal part of penis; at proximal end of penis with a blunt penis coecum; penial retractor runs from diaphragm to proximal part of epiphallus, which tapers proximally; flagellum moderately long, broadest in its middle part, without node, gradually tapering towards its proximal end; vas deferens narrow and long; vagina long; peduncle of bursa copulatrix subdivided into a broader, cylindrical distal part and a narrower, longer proximal part.

\section{Remarks}

Landouria nodifera sp. nov., L. tonywhitteni sp. nov., L. pacitanensis sp. nov., L. sukoliloensis sp. nov., L. sewuensis sp. nov. and L. intumescens share strongly depressed, brownish-corneous, sharply keeled shells. These species cannot be reliably distinguished by shell characters. Landouria pacitanensis sp. nov. shares a coecum at the proximal end of the penis with $L$. nodifera sp. nov., from which it differs in the long and very muscular distal part of the penis, which is separated from the proximal part of the penis by a very narrow constriction, the insertion of the penial retractor close to the distal end of the epiphallus and the lack of a node at the base of the flagellum.

The populations from the surroundings of Srau Beach differ in shell size and shape. The populations closer to the beach are smaller (D 10.6-12.7 $\mathrm{mm}$ vs $13.7-14.7 \mathrm{~mm}$ ) and more elevated (D/H 1.57-1.83 vs 1.70-2.18) than those from further inland.

\section{Distribution}

Landouria pacitanensis sp. nov. is endemic to the Pacitan Regency in East Java (Fig. 53). 
Landouria intumescens (Martens, 1867) comb. nov.

Figs 42, 53, 66, 72-74; Tables 3-4

Helix intumescens Martens, 1867: 263, pl. 13, fig. 10 ("bei Surabaya und dem benachbarten Grisse ... im Tengergebirge bei Wonosari”) (in part?).

Landouria winteriana - van Benthem Jutting 1950: 463 (in part). (not Pfeiffer, 1842)

\section{Diagnosis}

Landouria intumescens is characterized by a medium-sized, brownish-corneous, depressed conical, sharply keeled shell and a penis that is divided by a constriction into a long distal and a short proximal section and a flagellum with a broad, crenated distal part and a short and narrow proximal part.

\section{Material examined}

\section{Lectotype}

INDONESIA • lectotype (here designated); East Java, Surabaya; $7^{\circ} 20^{\prime} \mathrm{S}, 112^{\circ} 45^{\prime} \mathrm{E}$; E. von Martens leg.; measurements: $\mathrm{D}=13.4 \mathrm{~mm}, \mathrm{H}=8.2 \mathrm{~mm}$; ZMB 5160a.

\section{Paralectotypes}

INDONESIA • 7 spec.; same data as for lectotype; ZMB 5160.

\section{Other material}

INDONESIA - East Java 2 spec. (det. anat.); Tuban, Montong, Guwoterus, teak and agroforestry; $6^{\circ} 57^{\prime} 56^{\prime \prime}$ S, $111^{\circ} 47^{\prime} 56^{\prime \prime}$ E; 122 m a.s.1.; MZB 19208 • 11 spec. (det. anat.); Tuban, Montong, Guwoterus,

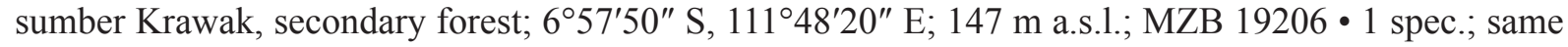
data as for preceding; MZB 19207 • 8 spec.; same data as for preceding; ZMH 133438 - 20 spec.; Bojonegoro, Dander, Sumberarum, mouth of Lawa Cave; $7^{\circ} 16^{\prime} 30^{\prime \prime} \mathrm{S}, 111^{\circ} 51^{\prime} 09^{\prime \prime}$ E; $117 \mathrm{~m}$ a.s.l.; MZB $19284 \bullet 10$ spec.; same data as for preceding; ZMH $1334110 \bullet 1$ spec.; Bojonegoro, Dander, Sumberarum, mouth of Sumur Cave; $7^{\circ} 16^{\prime} 08^{\prime \prime}$ S, 111 ${ }^{\circ} 51^{\prime} 41^{\prime \prime}$ E; $72 \mathrm{~m}$ a.s.1.; MZB $19263 \bullet 4$ spec.; same data as for preceding; MZB 19316 • 8 spec.; same data as for preceding; ZMH 133415.

\section{Description}

SHELl (Figs 72-74; Tables 3-4). Depressed conical, with 5-5.75 slightly convex whorls; protoconch almost smooth; teleoconch with fine, irregular wrinkles; apical side with indistinct incised spiral lines, umbilical side with more distinct spiral lines; without scaly processes; tuberculate around umbilicus; brownish-corneous, some with a light brownish band at periphery; body whorl sharply keeled; aperture rounded rhombic; upper insertion of peristome slightly descending; peristome expanded, reflexed and slightly thickened; umbilicus slightly eccentric, wide, comprising $22-34 \%$ of shell diameter, hardly obscured by columellar edge.

Genitalia (Figs 42, 66; Table 4). Atrium short; penis long, distal part cylindrical, broad, tapering proximally, separated from proximal part by a constriction before bend in penis, proximal part slightly dilated at its proximal end, without penis coecum; membranaceous sheath around distal part of penis connected with proximal part of epiphallus by tissue; penial retractor runs from diaphragm to middle part of short, cylindrical epiphallus; flagellum moderately long, broadest near its distal end, slightly crenated, without node, proximal part narrow, almost cylindrical; vas deferens narrow and long; vagina long, distally broad, proximally narrow; oviduct shorter, proximally tapering; peduncle of bursa copulatrix subdivided into a broader, cylindrical distal part and a narrower, longer proximal part, ending in an elongate oval bursa beside albumen gland. Right ommatophoral retractor runs between penis and vagina. 


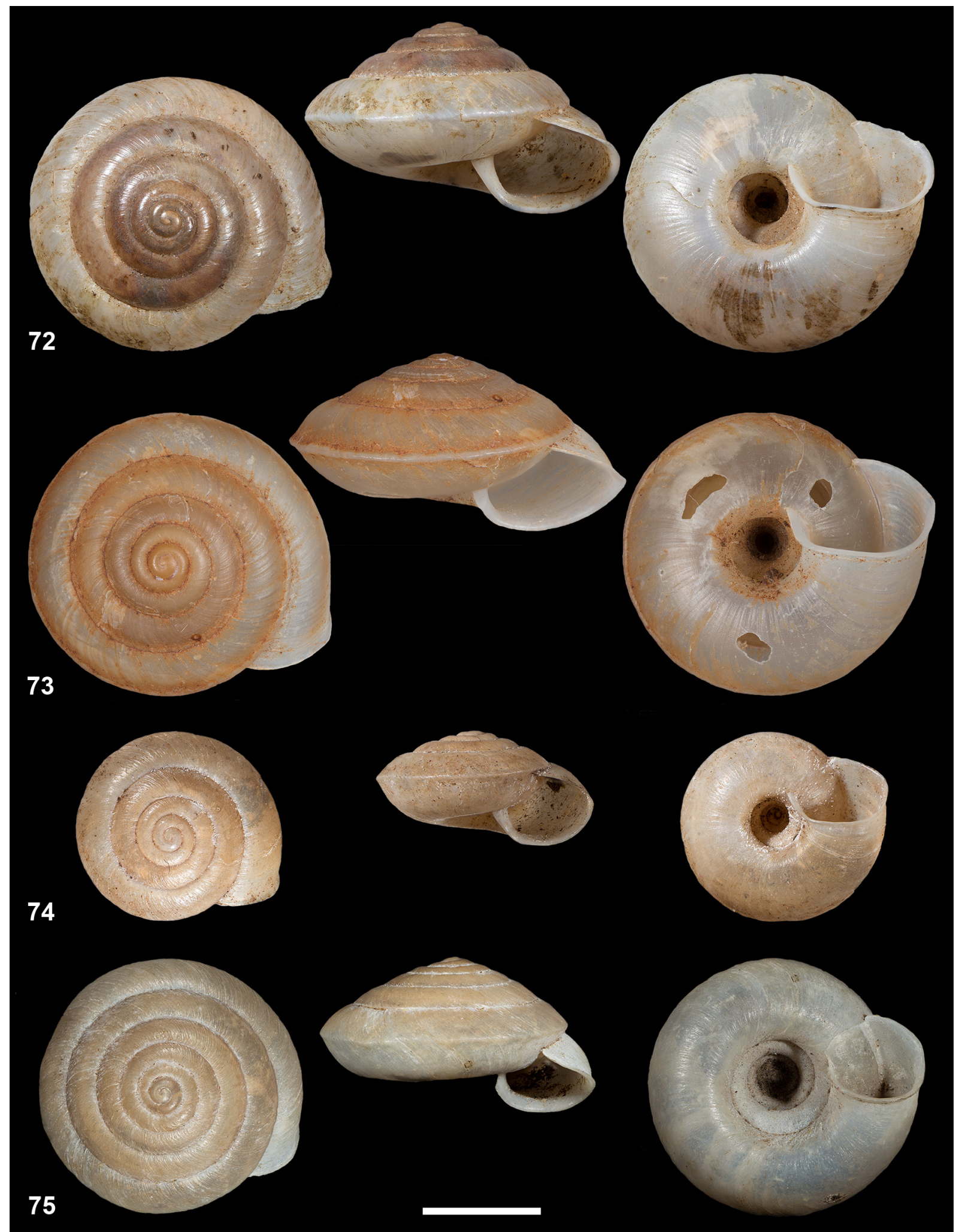

Figs 72-75. Shells of species of Landouria from Java. 72. L. intumescens (Martens, 1867), East Java, Surabaya (lectotype, ZMB 5160a). 73. L. intumescens, East Java, Guwoterus (MZB 19207). 74. L. intumescens?, East Java, Wonosari (paralectotype, ZMB 230704). 75. L. moussoniana (Martens, 1867), East Java, Wonosari (syntype, ZMB 109902). Scale bar: $5 \mathrm{~mm}$. 


\section{Remarks}

Martens (1867: 263, pl. 13, fig. 10) proposed the name H. intumescens for a medium-sized morph from Surabaya, which he figured, but also included a smaller morph from Wonosari in the Tengger Mountains in the nominal taxon. To fix the name for the morph from Surabaya, we designate the specimen figured by Martens (1867: pl. 13, fig. 10; see Fig. 72) as the lectotype.

We examined specimens from Tuban Regency anatomically; they agree in shell characters with the morph from Surabaya. The specific identity of these specimens with L. intumescens must be proven by an anatomical examination of specimens from Surabaya.

Whether the smaller paralectotypes of $H$. intumescens from Wonosari in the Tengger Mountains (ZMB 230704; Fig. 74) are conspecific with $L$. intumescens must also be proven by an anatomical examination.

Landouria intumescens cannot be reliably distinguished from $L$. winteriana by shell characters. However, the shells of $L$. intumescens usually differ from those of $L$. winteriana in the larger diameter (11.6$14.1 \mathrm{~mm}$ vs $9.7-12.6 \mathrm{~mm}$ in $L$. winteriana) and in the relatively narrower umbilicus, which comprises $22-34 \%$ of the shell diameter in L. intumescens vs $29-51 \%$ in L. winteriana. Landouria intumescens also differs from $L$. winteriana in the penis, which becomes narrow towards its proximal end, whereas it ends proximally in a globular dilatation in L. winteriana (Fig. 15).

\section{Distribution}

Landouria intumescens is known only from northern East Java (Fig. 53).

Landouria moussoniana (Martens, 1867) comb. nov.

Figs 55, 75; Tables 3-4

Helix sumatrana var. moussoniana Martens, 1867: 266 ("Oestliches Java, im Tengergebirge bei Wonosari").

Landouria rotatoria - van Benthem Jutting 1950: 461 (in part). (not Pfeiffer, 1842)

\section{Diagnosis}

Landouria moussoniana is characterized by a tightly coiled, keeled shell with a hardly eccentric, very wide umbilicus.

\section{Material examined}

Syntypes

INDONESIA • 6 spec.; East Java, Wonosari; 754’ S, 112²49’ E; ZMB 109902.

\section{Description}

SHELL (Fig. 75; Tables 3-4). Depressed conical, with 5-6.25 slightly convex whorls; protoconch almost smooth; teleoconch with irregular wrinkles; apical side with indistinct incised spiral lines, umbilical side with more distinct spiral lines; scaly processes not observed; tuberculate around umbilicus; brownishcorneous; body whorl keeled; aperture oval; upper insertion of peristome hardly or distinctly descending; peristome expanded, reflexed and slightly thickened; umbilicus very wide, comprising $37-38 \%$ of shell diameter, hardly obscured by columellar edge.

Genitalia. Unknown. 


\section{Remarks}

Martens (1867) recorded L. moussoniana from Wonosari in the Tengger Mountains together with L. smimensis and another form of Landouria that he included in his L. intumescens. Landouria moussoniana differs from $L$. intumescens in having a more tightly coiled, less sharply keeled shell with a wider umbilicus (U/D $0.37-0.38$ vs $0.22-0.34$ in L. intumescens).

\section{Distribution}

Landouria moussoniana is endemic to the Tengger Mountains in East Java (Fig. 55).

Landouria schepmani (Möllendorff, 1897) comb. nov.

Figs 54, 76; Tables 3-4

Plectotropis schepmani Möllendorff, 1897: 68 (“Java”, more exactly (van Benthem Jutting 1950; Zilch 1966): "Bungbulan, 1500 feet").

Landouria winteriana - van Benthem Jutting 1950: 463 (in part). — Zilch 1966: 294, pl. 7, fig. 7 (in part). (not Pfeiffer, 1842)

\section{Diagnosis}

Landouria schepmani is characterized by a depressed conical, sharply keeled shell with distinct incised spiral lines but no discernible scales, and a narrow umbilicus.

\section{Material examined}

\section{Lectotype}

INDONESIA - lectotype (designated by Zilch 1966); West Java, Sukapura, Gunung Bungbulang; $7^{\circ} 27^{\prime}$ S, $107^{\circ} 36^{\prime}$ E?; ca 450 m a.s.l.; SMF 8877.

\section{Paralectotype}

INDONESIA • 1 spec.; same data as for lectotype; SMF 8878.

\section{Description}

SHeLL (Fig. 76; Tables 3-4). Depressed conical, with 5-5.25 slightly convex whorls; protoconch almost smooth; teleoconch with fine, irregular wrinkles and distinct incised spiral lines; no scales discernible; tuberculate around umbilicus; brownish-corneous; body whorl sharply keeled; aperture rounded rhombic; upper insertion of peristome slightly descending; peristome expanded, reflexed and slightly thickened; umbilicus slightly eccentric, wide, comprising $21 \%$ of shell diameter, hardly obscured by columellar edge.

Genitalia. Unknown.

\section{Remarks}

Van Benthem Jutting (1950) and Zilch (1966) synonymized L. schepmani with L. winteriana. However, L. schepmani differs from $L$. winteriana in having a sharply keeled, more conical shell with distinct incised spiral lines and a narrower umbilicus (U/D 0.21 vs $0.29-0.51$ in L. winteriana).

According to the original label, the type locality is Sukapura, Gunung Bungbulan. Van Benthem Jutting (1950: 465) listed a Bungbulan south of Gunung Papandayan ( $\left.7^{\circ} 27^{\prime} \mathrm{S}, 107^{\circ} 36^{\prime} \mathrm{E}\right)$. It is not clear whether this is the Bungbulan of Fruhstorfer, because there are also several other localities called Bungbulan in the former Sukapura Regency. For example, there is a Gunung Bungbulang, now called Gunung 


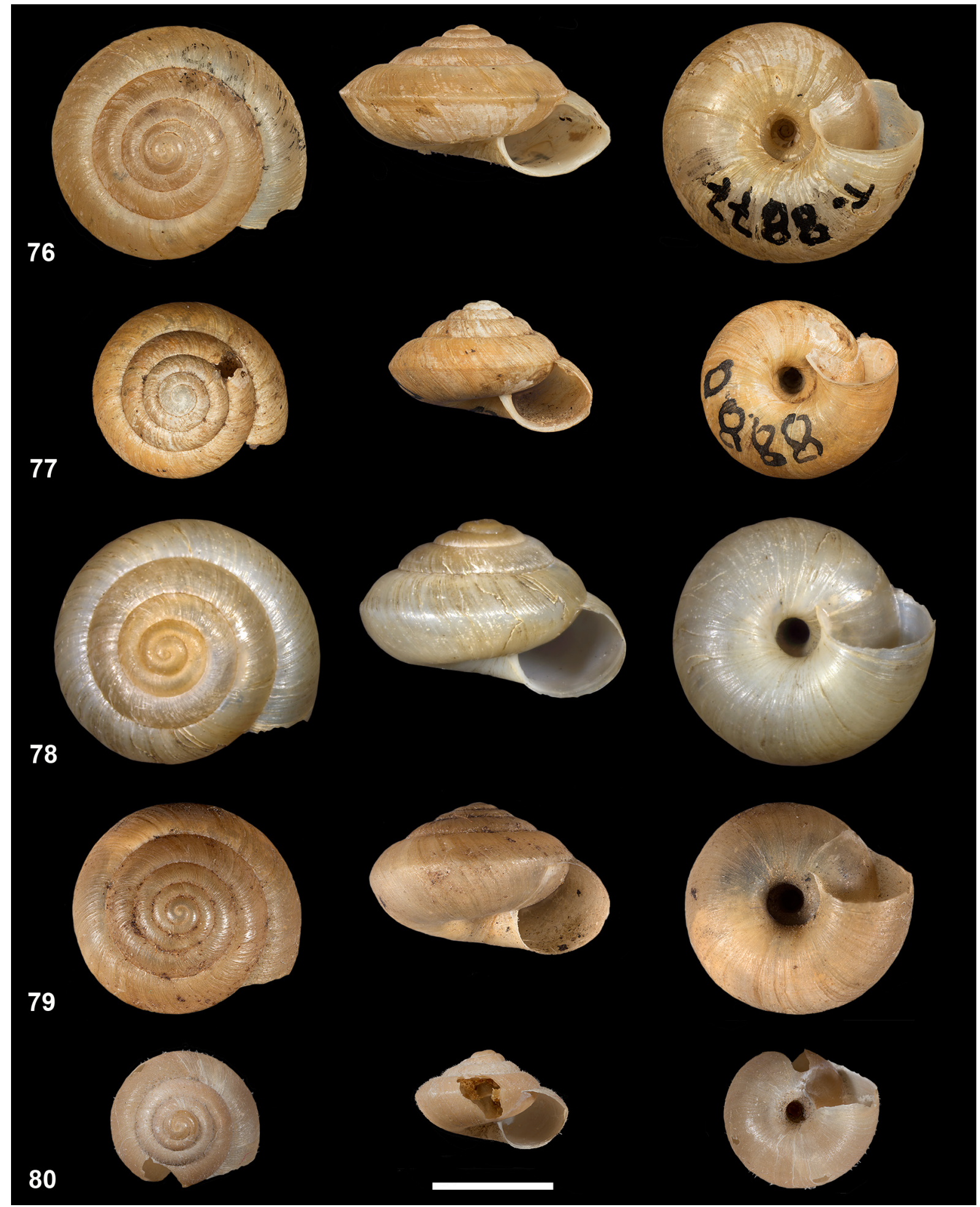

Figs 76-80. Shells of species of Landouria from Java. 76. L. schepmani (Möllendorff, 1897), West Java, Sukapura, Gunung Bungbulang (lectotype, SMF 8877). 77. L. monticola van Benthem Jutting, 1950, West Java, Gunung Cikuray (paratype, SMF 8880). 78. L. smimensis (Mousson, 1848), East Java, Gunung Semeru, ca 1200 m a.s.1., $8^{\circ} 06^{\prime}$ S, 112 55' E (syntype of Helix smimensis, ZMZ 508598). 79. L. smimensis, East Java, Nongkojajar (holotype of Plectotropis tenggerica Schepman, 1912, ZMA 174453). 80. L. smimensis, East Java, Tosari (ZMA 390881). Scale bar: 5 mm. 
Bangkuang, in West Java at $7^{\circ} 12^{\prime} \mathrm{S}, 108^{\circ} 05^{\prime} \mathrm{E}$. Thus, the distribution area of $L$. schepmani remains dubious.

\section{Distribution}

Landouria schepmani is known only from "Gunung Bungbulan" in West Java (Fig. 54; see Remarks).

Landouria monticola van Benthem Jutting, 1950

Figs 10, 55, 77; Tables 3-4

Landouria monticola van Benthem Jutting, 1950: 469, fig. 85 ("Mt. Tjikorai, 2500-2800 m").

Landouria monticola - Zilch 1966: 294.

\section{Diagnosis}

Landouria monticola is characterized by a conical, keeled shell with distinct incised spiral lines, but no discernible scales, and a narrow umbilicus.

\section{Material examined}

Type material

INDONESIA $\bullet 2$ paratypes; Java; SMF 8879. - West Java 3 paratypes; Gunung Cikuray; $7^{\circ} 19^{\prime}$ S, $107^{\circ} 52^{\prime} \mathrm{E}$; $2500 \mathrm{~m}$ a.s.l.; SMF $8880 \bullet 1$ paratype (or holotype?); Gunung Cikuray, summit; $7^{\circ} 19^{\prime} \mathrm{S}$, $107^{\circ} 52^{\prime} \mathrm{E} ; 2800 \mathrm{~m}$ a.s.1.; ZMA 407615.

\section{Other material}

INDONESIA • 1 spec.; West Java, Gunung Cikuray; 7¹9' S, 10752' E; 2400 m a.s.1.; ZMB 46038.

\section{Description}

SHELl (Figs 10, 77; Tables 3-4). Conical, with 4.5-5.25 convex whorls; protoconch almost smooth; teleoconch with irregular wrinkles and distinct incised spiral lines; no scales discernible; not tuberculate around umbilicus; yellowish or brownish-corneous; body whorl keeled; aperture oval; upper insertion of peristome hardly descending; peristome slightly expanded, not reflexed and slightly thickened; umbilicus hardly eccentric, narrow, comprising $20-25 \%$ of shell diameter, hardly obscured by columellar edge.

Genitalia. Unknown.

\section{Remarks}

Landouria monticola resembles L. schepmani (Fig. 76), in which it was apparently included by Möllendorff (1897). It differs from that species in the smaller (D 8.5-9.5 mm vs $10.5-10.9 \mathrm{~mm}$ in L. schepmani), higher conical (D/H 1.49-1.60 vs 1.72-1.76 in L. schepmani), less sharply keeled shell.

The holotype of L. monticola could not be traced in the RMNH/ZMA collection. Van Benthem Jutting (1950) reported L. monticola from several regions in West, Central and East Java. We examined a juvenile paratype of L. monticola from Gunung Kawi in East Java (ZMA 137804), which differs from the paratypes from Gunung Cikuray in the smoother shell with a narrower umbilicus. We doubt that this specimen is conspecific with the paratypes of L. monticola from Gunung Cikuray. The identity of the populations from high mountain regions in Central Java with L. monticola must also be checked. 


\section{Distribution}

Currently only the populations from Gunung Cikuray in West Java are included in L. monticola (Fig. 55). Records from other regions of Java (van Benthem Jutting 1950) and from Sumatra (Heryanto 2013) probably refer to other species.

Landouria smimensis (Mousson, 1848)

Figs 11, 43, 52, 67, 78-80; Tables 3-4

Helix smimensis Mousson, 1848: 266 (“Java”; more exactly (Mousson 1849): “Smim, 4000"”).

Helix smironensis Mousson, 1850: 177 (unjustified emendation).

Helix smiruensis Martens, 1867: 268 (unjustified emendation).

Plectotropis tenggerica Schepman, 1912: 233, pl. 10, figs 9-11 (“Nongkodjadjar”).

Helix smimensis - Mousson 1849: 21, pl. 2, fig. 10.

Plectotropis sp. - Schepman 1915: 19.

Landouria smironensis - van Benthem Jutting 1950: 467 (in part).

\section{Diagnosis}

Landouria smimensis is characterized by a small, depressed conical shell with hairs and a usually slightly angular body whorl, a long penis, which gradually tapers towards its proximal end, and a slightly crenated, gradually tapering flagellum.

\section{Material examined}

Type material

INDONESIA - East Java • holotype of P. tenggerica; Nongkojajar; $7^{\circ} 54^{\prime}$ S, $112^{\circ} 49^{\prime}$ E; ZMA 174453 • 2 syntypes of $H$. smimensis; Gunung Semeru; 806' S, 112 ${ }^{\circ} 55^{\prime}$ E; ca 1200 m a.s.1., ZMZ 508598.

\section{Other material}

INDONESIA • 23 spec. (vouchers for Martens 1867); Java; ZMB $4062 \cdot 1$ spec. (voucher for van Benthem Jutting 1950); Java; ZMB 67043. - East Java • 3 spec. (vouchers for van Benthem Jutting 1950); Nongkojajar, near waterfall; $7^{\circ} 54^{\prime} \mathrm{S}, 112^{\circ} 49^{\prime} \mathrm{E}$; ZMA $407685 \cdot$ many spec. (vouchers for van Benthem Jutting 1950); same data as for preceding; ZMA $407681 \cdot 7$ spec.; Nongkojajar, near Baron; $7^{\circ} 54^{\prime} \mathrm{S}, 112^{\circ} 49^{\prime} \mathrm{E}$; ZMA $407686 \bullet 2$ spec. (vouchers for van Benthem Jutting 1950) (det. anat.); Tosari; $7^{\circ} 53^{\prime} \mathrm{S}, 112^{\circ} 54^{\prime} \mathrm{E}$; ca 1800 m a.s.1.; ZMA 390881.

\section{Description}

SHELl (Figs 11, 78-80; Tables 3-4). Depressed conical, with 4.5-5.5 convex whorls; protoconch almost smooth; teleoconch irregularly striated, with indistinct incised spiral lines; with hair-like processes; not tuberculate around umbilicus; brownish-corneous; body whorl slightly angular or rounded; aperture oval; upper insertion of peristome hardly descending; peristome sharp; umbilicus slightly eccentric, narrow, comprising $18-32 \%$ of shell diameter, hardly obscured by columellar edge.

Genitalia (Figs 43, 67; Table 4). Atrium short; penis long, gradually tapering towards its proximal end; without penis coecum; surrounded by a membranaceous sheath connected to proximal epiphallus by tissue; penial retractor runs from diaphragm to middle part of cylindrical epiphallus; flagellum moderately long, narrow, broadest in its middle part, without node, gradually tapering towards its proximal end, slightly crenated; vas deferens narrow and long; vagina broad and short; oviduct short, proximally slightly tapering; peduncle of bursa copulatrix subdivided into a broad abruptly tapering distal part and a narrower and longer proximal part, ending in an elongate oval bursa beside albumen gland. Right ommatophoral retractor runs between penis and vagina. 


\title{
Remarks
}

The original spelling of the species name was Helix smimensis Mousson, 1848. This was not an inadvertent error, such as a lapsus calami or printer's error, because this spelling was also used later by Mousson (1849). Thus, H. smimensis is the correct original spelling according to ICZN, Article 32 . The name was intentionally changed to $H$. smironensis by Mousson (1850) and to H. smiruensis by Martens (1867). Both names are unjustified emendations, which are available with these authors and dates, and which are junior objective synonyms of $H$. smimensis (ICZN, Art. 33). According to ICZN, Article 33.2.3.1, an unjustified emendation may be deemed to be a justified emendation, if it is in prevailing usage. Helix smiruensis was used by Tryon (1887), Leschke (1914) and Rensch (1932), L. smironensis by van Benthem Jutting (1950) and Vermeulen \& Whitten (1998), the incorrect subsequent spelling Landouria cf. smiroensis by Boonngam et al. (2008) and the correct original spelling L. smimensis by Köhler et al. (in press). Thus, there is no prevailing usage of any of the emendations and the correct original spelling should be used.

The voucher specimen for L. tenggerica of Rensch (1934) from Gunung Lawu, $2400 \mathrm{~m}$ a.s.l. (ZMB 76086) is tuberculate around the umbilicus and is a juvenile of a different species.

\section{Distribution}

Landouria smimensis is known from the Tengger Mountains in East Java (Fig. 52). It has also been recorded from other mountains in Central and East Java (Rensch 1934), Lombok (Rensch 1932), Bali (Vermeulen \& Whitten 1998) and Thailand (Boonngam et al. 2008), but these populations probably represent separate species.

Landouria leucochila (Gude, 1905) comb. nov.

Fig. 81; Tables 3-4

Plectotropis leucochila Gude, 1905: 14, pl. 3, figs 1a-c ("Java").

Landouria rotatoria - van Benthem Jutting 1950: 461 (in part). (not Pfeiffer, 1842)

\section{Diagnosis}

Landouria leucochila is characterized by a large, depressed conical, yellowish shell with an angular body whorl, a wide umbilicus and scales all over the teleoconch.

\author{
Material examined \\ Holotype \\ INDONESIA • holotype; Java; NHM 1922.8.29.87.
}

\section{Description}

SHELL (Fig. 81; Tables 3-4). Depressed conical, with 5.25 hardly convex whorls; protoconch almost smooth; teleoconch with irregular wrinkles; apical side with indistinct incised spiral lines, umbilical side with more distinct spiral lines; scaly processes all over teleoconch; tuberculate around umbilicus;

Figs 81-84 (opposite page). Shells of species of Landouria from Java. 81. L. leucochila (Gude, 1905), Java (holotype, NHM 1922.8.29.87). 82. L. zonifera sp. nov., Yogyakarta, Kulonprogo, Sibolong (holotype, MZB 17727). 83. L. ciliocincta (Möllendorff, 1897), East Java, Zuider Mountains (lectotype, SMF 9176a). 84. L. ciliocincta, East Java, Malang, Kondangmerak, protected forest (MZB 19211). Scale bar: $5 \mathrm{~mm}$. 


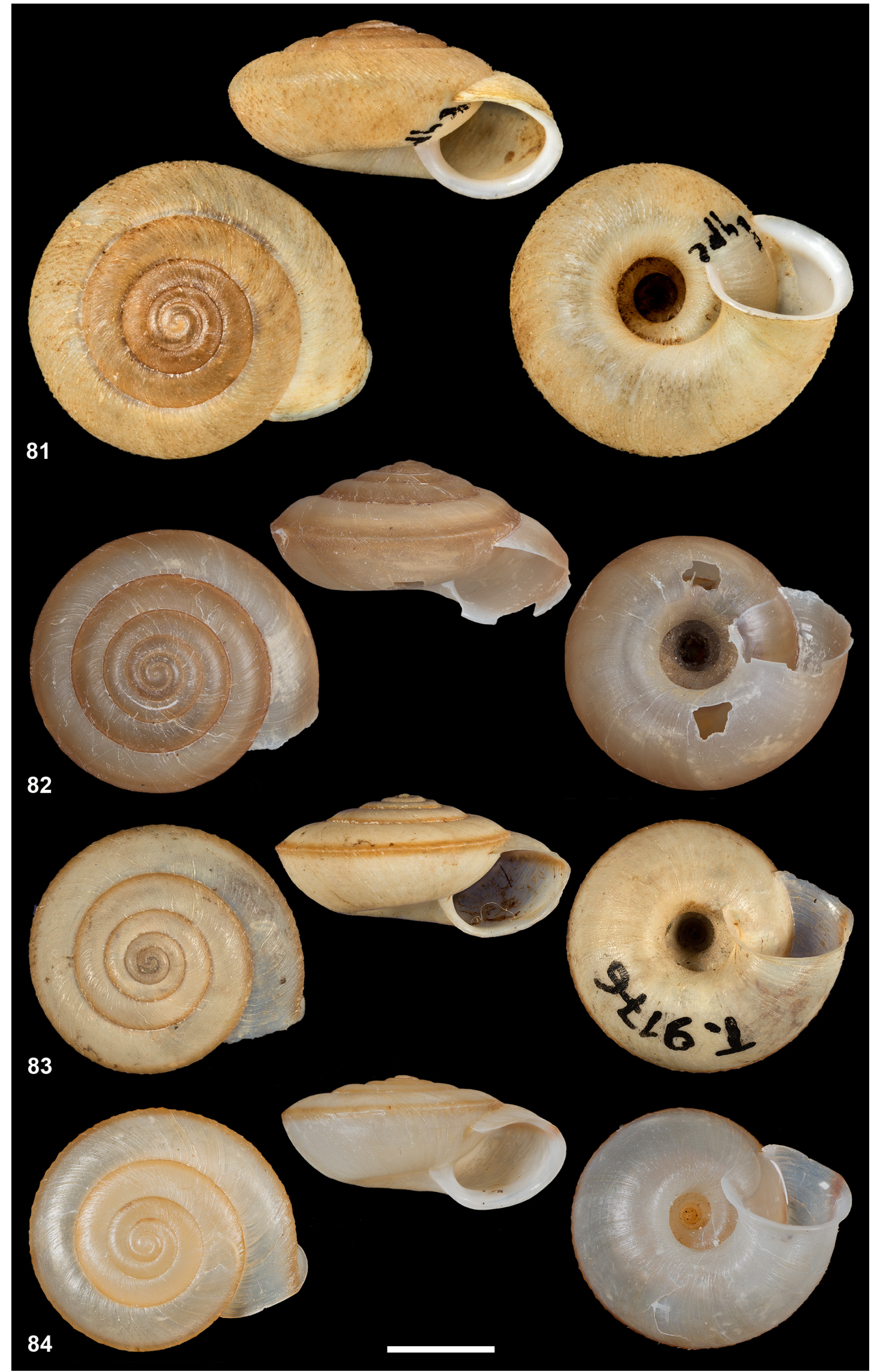


yellowish; body whorl angular; aperture oval; upper insertion of peristome descending; peristome expanded, reflexed and distinctly thickened; umbilicus eccentric, wide, comprising $30 \%$ of shell diameter, hardly obscured by columellar edge.

Genitalia. Unknown.

\title{
Distribution
}

The type locality and the distribution of L. leucochila in Java remain unknown.

\author{
Landouria zonifera sp. nov. \\ urn:1sid:zoobank.org:act:81A2A657-B12B-43BE-B19D-22AE36E2525C
}

Figs 44, 54, 68, 82; Tables 3-4

\section{Diagnosis}

Landouria zonifera sp. nov. is characterized by a keeled, corneous shell with a brownish zone at the periphery, a wide umbilicus and a flagellum that is more than twice as long as the short epiphallus.

\section{Etymology}

Landouria zonifera sp. nov. is named after the brownish zone at its shell periphery (Latin: 'zona' = 'zone', 'ferre' = 'to carry'; used as an adjective).

\section{Material examined}

\section{Holotype}

INDONESIA - holotype (det. anat.); Yogyakarta, Kulonprogo, Sibolong, snakefruit plantation; $7^{\circ} 44^{\prime} 44^{\prime \prime}$ S, $110^{\circ} 08^{\prime} 02^{\prime \prime}$ E; 716 m a.s.1.; 9 Jan. 2013; A.S. Nurinsiyah and T. Setiadi leg.; D = $13.2 \mathrm{~mm}$, $\mathrm{H}=7.6 \mathrm{~mm}$; MZB 17727 .

\section{Paratypes}

INDONESIA - 1 spec.; Yogyakarta, Kulonprogo, Sibolong, Sibolong Hill, agroforestry; $7^{\circ} 45^{\prime} 00^{\prime \prime}$ S, $110^{\circ} 07^{\prime} 00^{\prime \prime} \mathrm{E} ; 721 \mathrm{~m}$ a.s.1.; MZB $17762 \bullet 13$ spec.; same data as for holotype; MZB $17720 \bullet 1 \mathrm{spec}$; same data as for holotype; ZMH $133420 \bullet 4$ spec.; Kulonprogo, Jonggrangan; $7^{\circ} 44^{\prime} 20^{\prime \prime}$ S, $110^{\circ} 08^{\prime} 32^{\prime \prime}$ E; 730 m a.s.l.; ZMH 140031.

\section{Description}

SHELL (Fig. 82; Tables 3-4). Depressed conical, with 5.5 -5.75 hardly convex whorls; protoconch almost smooth; teleoconch with fine, irregular wrinkles and indistinct incised spiral lines; scaly processes sparse, mainly at periphery; tuberculate around umbilicus; corneous with a brownish zone at periphery; body whorl keeled; aperture rounded rhombic; upper insertion of peristome slightly descending; peristome expanded, reflexed and slightly thickened; umbilicus slightly eccentric, wide, comprising $40 \%$ of shell diameter, hardly obscured by columellar edge.

Genitalia (Figs 44, 68; Table 4). Atrium short; penis long, cylindrical, slightly dilated at proximal end, without penis coecum; membranaceous sheath around distal part of penis connected with proximal part of epiphallus by tissue; penial retractor runs from diaphragm to proximal part of short, cylindrical epiphallus; flagellum more than twice as long as epiphallus, broadest near its proximal end, then abruptly tapering, slightly crenated, without node; vas deferens narrow and long; vagina very long, cylindrical; oviduct shorter, broad, proximally tapering; peduncle of bursa copulatrix subdivided into a broader, 
cylindrical distal part and a narrower, longer proximal part, ending in an elongate oval bursa beside albumen gland. Right ommatophoral retractor runs between penis and vagina.

\section{Remarks}

Landouria zonifera sp. nov. clusters in the molecular phylogeny (Fig. 1) with L. abdidalem sp. nov. (Fig. 56) and L. tholiformis sp. nov. (Fig. 51). Landouria zonifera sp. nov. and L. abdidalem sp. nov. have overlapping ranges. Landouria zonifera sp. nov. differs from L. abdidalem sp. nov. in the smaller (D $12.5-13.2 \mathrm{~mm}$ vs $13.1-16.4 \mathrm{~mm}$ in L. abdidalem sp. nov.), more depressed (D/H 1.73-1.81 vs 1.93-2.14 in L. abdidalem sp. nov.), corneous shell with a brownish zone at the periphery, the shorter penis and a flagellum that is more than twice as long as the short epiphallus. Furthermore, Landouria zonifera sp. nov. occurs sympatrically with $L$. menorehensis sp. nov. (Fig. 88) from which it can easily be distinguished by the keeled, depressed conical, corneous shell with a brownish zone at the periphery.

\section{Distribution}

Landouria zonifera sp. nov. is endemic to the Menoreh karst area (Fig. 54).

Landouria ciliocincta (Möllendorff, 1897)

Figs 45, 54, 69, 83-84; Tables 3-4

Plectotropis ciliocincta Möllendorff, 1897: 67 (“Java”; more exactly (Zilch 1966): “O-Java: ZuiderGebirge").

Landouria ciliocincta - van Benthem Jutting 1950: 466 (in part). — Zilch 1966: 293, pl. 7, fig. 1 (in part). — Dharma 2015: 8, figs 2, 4 (in part). — Nurinsiyah et al. 2016: 559.

\section{Diagnosis}

Landouria ciliocincta is characterized by a whitish, almost disc-like, keeled shell with an only slightly elevated spire, a reddish-brown band at the angular periphery and a wide umbilicus, a long, cylindrical penis with a small bulge at its proximal end, and a short, very broad flagellum, which tapers towards its proximal end.

\section{Material examined}

Lectotype

INDONESIA - lectotype (designated by Zilch 1966); East Java, Zuider (Southern) Mountains; SMF 9176a.

\section{Paralectotype}

INDONESIA 1 spec.; same data as for lectotype; SMF $9716 \mathrm{~b}$.

\section{Other material}

INDONESIA - East Java 1 spec. (voucher for Dharma 2015); Malang, Ngliyep; $8^{\circ} 23^{\prime} \mathrm{S}, 112^{\circ} 25^{\prime} \mathrm{E}$; near sea level; BDJ 1 spec. (det. anat.) (voucher for Nurinsiyah et al. 2016); Malang, Kondangmerak,

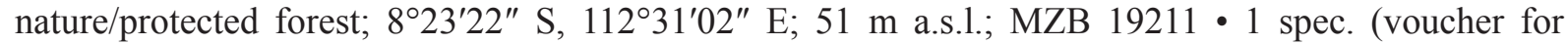
Nurinsiyah et al. 2016); Malang, Kondangmerak, nature/protected forest; $8^{\circ} 23^{\prime} 21^{\prime \prime} \mathrm{S}, 112^{\circ} 31^{\prime} 05^{\prime \prime} \mathrm{E}$; 100 m a.s.l.; MZB 17645 • 2 spec.; Tengger Mountains; ZMB 45408.

\section{Description}

SHELl (Figs 83-84; Tables 3-4). Almost disc-like with slightly elevated spire, with 5-6 moderately convex whorls; protoconch almost smooth; teleoconch with fine, irregular wrinkles, indistinct incised 
spiral lines and sparse scaly processes, mainly at periphery; tuberculate around umbilicus; whitishcorneous with a brownish band at periphery; body whorl keeled; aperture rounded rhombic; upper insertion of peristome descending; peristome expanded, reflexed and thickened; umbilicus slightly eccentric, wide, comprising $22-30 \%$ of shell diameter, hardly obscured by columellar edge.

Genitalia (Figs 45, 69; Table 4). Atrium short; penis long, cylindrical, with a small bulge at its proximal end, without penis coecum; membranaceous sheath around distal part of penis connected with flagellum by tissue; penial retractor runs from diaphragm to middle part of cylindrical epiphallus; flagellum short, very broad, without node, tapering towards its proximal end; vas deferens narrow and long; vagina long; oviduct short, broad, proximally tapering; peduncle of bursa copulatrix subdivided into a broad, proximally tapering distal part and a narrower, longer proximal part, ending in an elongate oval bursa beside albumen gland. Right ommatophoral retractor runs between penis and vagina.

\section{Remarks}

Landouria ciliocincta shares with L. pakidulan sp. nov. and L. menorehensis sp. nov. the whitish shell with a brown band at the periphery. For differences, see remarks under L. pakidulan sp. nov.

\section{Distribution}

Landouria ciliocincta is known from Malang Regency in East Java (Fig. 54). Records from other areas of Java (Oostingh 1923; van Benthem Jutting 1941, 1950; Zilch 1966; Dharma 2015) and from Sumatra (van Benthem Jutting 1959; Heryanto 2013) probably refer to other species.

Landouria pakidulan sp. nov. urn:1sid:zoobank.org:act:D059D3B1-85D9-4760-B9F2-ABC24A7735ED

Figs 46, 54, 70, 85; Tables 3-4

Landouria ciliocincta - van Benthem Jutting 1941: 310 (in part); 1950: 466 (in part). — Dharma 2015: 8 (in part). (not Möllendorff, 1897)

\section{Diagnosis}

Landouria pakidulan sp. nov. is characterized by a whitish, depressed conical shell with a reddish-brown band at the angular periphery and a wide umbilicus, a short penis with a large bulge at the proximal end and a moderately long, almost smooth flagellum, which tapers abruptly near its proximal end.

\section{Etymology}

Landouria pakidulan sp. nov. (from the Sundanese and Javanese word 'pakidulan' meaning 'southern') is named after its occurrence in southern Java.

\section{Material examined}

Holotype

INDONESIA - holotype (det. anat.) (voucher for L. ciliocincta in van Benthem Jutting 1941, 1950); Central Java, Nusa Kambangan Island, Nature Monument Gligir; 744' S, 108 $53^{\prime}$ E; 15.-16 Feb. 1927; P.F. Franck leg.; $\mathrm{D}=12.5 \mathrm{~mm}, \mathrm{H}=6.9 \mathrm{~mm}$; ZMA 417011a.

\section{Paratypes}

INDONESIA - West Java • 2 spec.; Pangandaran, Green Canyon; 7²4' S, $108^{\circ} 27^{\prime}$ E; MZB $14067 \bullet$ 1 spec.; Pangandaran Nature Reserve; $7^{\circ} 42^{\prime}$ S, 108 $39^{\prime}$ E; MZB 6162. - Central Java • 2 spec. (vouchers for L. ciliocincta in van Benthem Jutting 1941, 1950); same data as for holotype; ZMA 417011. 


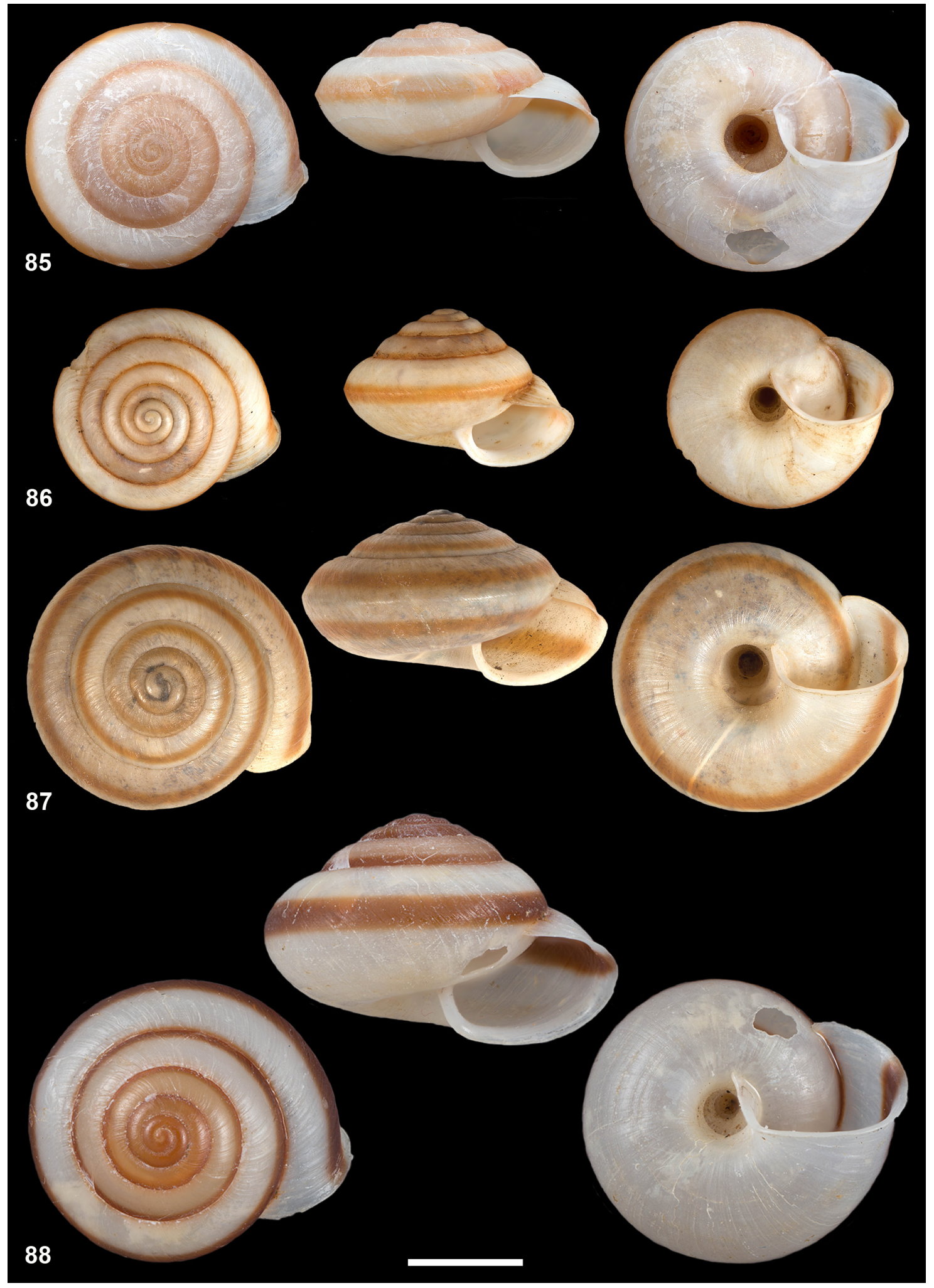

Figs 85-88. Shells of species of Landouria from Java. 85. L. pakidulan sp. nov., Central Java, Nusa Kambangan Island, nature monument Gligir (holotype, ZMA 417011a). 86. L. dharmai sp. nov., East Java, Tulungagung, Popoh (holotype, MZB 21272). 87. L. davini Dharma, 2015, Central Java, Baturraden, Pancuran Tujuh, Gunung Slamet (holotype, MZB 17063). 88. L. menorehensis sp. nov., Yogyakarta, Kulonprogo, Sibolong (holotype, MZB 19201). Scale bar: $5 \mathrm{~mm}$. 


\title{
Description
}

SHELL (Fig. 85; Tables 3-4). Depressed conical, with 5.25-5.5 slightly convex whorls; protoconch almost smooth; teleoconch with fine, irregular wrinkles; apical side with indistinct incised spiral lines, umbilical side with more distinct spiral lines; scaly processes not observed; tuberculate around umbilicus; whitish with a reddish-brown band at periphery; body whorl angular; aperture oval; upper insertion of peristome slightly descending; peristome expanded, reflexed and thickened; umbilicus eccentric, wide, comprising $24-30 \%$ of shell diameter, hardly obscured by columellar edge.

Genitalia (Figs 46, 70; Table 4). Atrium short; penis short, almost cylindrical, with a large bulge at proximal end, without penis coecum; membranaceous sheath around distal part of penis; penial retractor not observed; epiphallus cylindrical, short; flagellum moderately long, broadest in its middle part, abruptly tapering near its proximal end, almost smooth, without node; vas deferens narrow and long; vagina long; oviduct short, broad, proximally tapering; peduncle of bursa copulatrix subdivided into a broad, abruptly tapering distal part and a narrower, longer proximal part. Right ommatophoral retractor runs between penis and vagina.

\section{Remarks}

Landouria pakidulan sp. nov. shares with L. menorehensis sp. nov. and L. ciliocincta the whitish shell with a brown band at the periphery. Landouria pakidulan sp. nov. differs from L. menorehensis sp. nov. in the more depressed shell (D/H 1.70-1.86 vs 1.31-1.51 in L. menorehensis sp. nov.) with an angular body whorl and a wider umbilicus that comprises $24-30 \%$ of the shell diameter (vs $16-24 \%$ in L. menorehensis sp. nov.). The holotype and only anatomically examined specimen of Landouria pakidulan sp. nov. (Figs 70, 85) differs from L. menorehensis sp. nov. (Figs 71, 88) in the triangular dilation at the proximal end of the penis. More specimens must be examined to corroborate that this is a species specific character and not an individual aberration. Landouria pakidulan sp. nov. differs from L. ciliocincta in the less depressed shell (D/H 1.70-1.86 vs 1.86-2.12 in L. ciliocincta), the shorter penis and epiphallus, and the longer flagellum.

\section{Distribution}

Landouria pakidulan sp. nov. is known from southern Java from Pangandaran in West Java and Nusa Kambangan Island in Central Java (Fig. 54).

\author{
Landouria dharmai sp. nov. \\ urn:lsid:zoobank.org:act:B4620BC6-38C3-4E3D-AA72-46B82D2D1CB8
}

Figs 55, 86; Tables 3-4

Landouria ciliocincta - Dharma 2015: 8, pl. 1, fig. 3 (in part). (not Möllendorff, 1897)

\section{Diagnosis}

Landouria dharmai sp. nov. is characterized by a conical, whitish shell with a reddish-brown band at the angular periphery and a moderately wide umbilicus.

\section{Etymology}

Landouria dharmai sp. nov. is named in honour of the Indonesian malacologist Bunjamin Dharma, who discovered this species.

\section{Material examined}

Holotype

INDONESIA • holotype (voucher for L. ciliocincta in Dharma 2015: pl. 1, fig. 3); East Java, Tulungagung, Popoh; $8^{\circ} 16^{\prime}$ S, $111^{\circ} 48^{\prime}$ E; B. Dharma leg.; MZB 21272. 


\section{Description}

SHELl (Fig. 86; Tables 3-4). Conical, with 5.5 slightly convex whorls; protoconch almost smooth; teleoconch with fine, irregular wrinkles; apical side with indistinct incised spiral lines, umbilical side with more distinct spiral lines; scaly processes not observed; tuberculate around umbilicus; whitish with a reddish-brown band at periphery; body whorl angular; aperture oval; upper insertion of peristome descending; peristome expanded, reflexed and slightly thickened; umbilicus slightly eccentric, moderately wide, comprising $18 \%$ of shell diameter, hardly obscured by columellar edge.

Genitalia. Unknown.

\section{Remarks}

Landouria dharmai sp. nov. differs from L. ciliocincta (Figs 83-84), in which it was included by Dharma (2015), in the smaller (D $9.9 \mathrm{~mm}$ vs 12.4-15.6 $\mathrm{mm}$ in L. ciliocincta), higher conical (D/H 1.39 vs 1.862.12 in $L$. ciliocincta), less sharply keeled shell with a narrower umbilicus (U/D 0.18 vs $0.24-0.30$ in L. ciliocincta).

\section{Distribution}

Landouria dharmai sp. nov. is endemic to Tulungagung Regency in East Java (Fig. 55).

Landouria davini Dharma, 2015

Figs 55, 87; Tables 3-4

Landouria davini Dharma, 2015: 9, figs 5-7 ("Pancuran Tujuh, Baturraden, Mt. Slamet, Central Jawa, $720 \mathrm{~m}$ alt.”).

\section{Diagnosis}

Landouria davini is characterized by a depressed conical, whitish shell with one brown band above and one below the angular periphery.

\section{Material examined}

Holotype

INDONESIA - holotype; Central Java, Baturraden, Pancuran Tujuh, Gunung Slamet; $7^{\circ} 18^{\prime} 36^{\prime \prime}$ S, $109^{\circ} 13^{\prime} 05^{\prime \prime} \mathrm{E} ; 720 \mathrm{~m}$ a.s.1.; MZB 17063.

\section{Paratypes}

INDONESIA - Central Java • 1 spec.; Pondok Pakis, Bojong Tegal, Gunung Slamet; 7¹1'56" S, $109^{\circ} 11^{\prime} 56^{\prime \prime}$ E; 2200 m a.s.1.; BDJ • 1 spec.; Pondok Buncis, Bojong Tegal, Gunung Slamet; $7^{\circ} 13^{\prime} 04^{\prime \prime}$ S, $109^{\circ} 12^{\prime} 00^{\prime \prime}$ E; $1600 \mathrm{~m}$ a.s.l.; MZB 17066 • 3 spec.; same data as for holotype; MZB $17064 \cdot 3$ spec.; same data as for holotype; SMF $341605 \bullet 3$ spec.; same data as for holotype; RMNH $329337 \cdot 3$ spec.; same data as for holotype; BDJ • 1 spec.; Baturraden, Perhutani, Gunung Slamet; $7^{\circ} 18^{\prime} \mathrm{S}, 109^{\circ} 14^{\prime} \mathrm{E}$; 800 m a.s.1.; MZB 17065.

\section{Other material}

INDONESIA • 1 spec.; Central Java, Gunung Slamet; MZB 15425.

\section{Description}

SHELl (Fig. 87; Tables 3-4). Depressed conical, with 5-5.5 slightly convex whorls; protoconch almost smooth; teleoconch with fine, irregular wrinkles; apical side with indistinct incised spiral lines, umbilical side with more distinct spiral lines; scaly processes not observed; tuberculate around umbilicus; whitish- 
corneous with one reddish-brown band or a broader brownish zone above and one below periphery; body whorl angular; aperture oval; upper insertion of peristome slightly descending; peristome expanded, reflexed and slightly thickened; umbilicus slightly eccentric, moderately wide, comprising $29 \%$ of shell diameter, hardly obscured by columellar edge.

Genitalia. Unknown.

\section{Distribution}

Landouria davini is endemic to Gunung Slamet (800-2200 m a.s.1.) in Central Java (Fig. 55).

Landouria menorehensis sp. nov. urn:1sid:zoobank.org:act:E09F6255-8F7A-49E7-A3BC-E5B795EE2015

Figs 47, 55, 71, 88; Tables 3-4

\section{Diagnosis}

Landouria menorehensis sp. nov. is characterized by a whitish, globose shell with a distinct brown band above the rounded periphery and a moderately narrow umbilicus.

\section{Etymology}

Landouria menorehensis sp. nov. is named after its occurrence in the Menoreh karst area.

\section{Material examined}

\section{Holotype}

INDONESIA - holotype (det. anat.); Yogyakarta, Kulonprogo, Sibolong, agroforestry; $7^{\circ} 44^{\prime} 52^{\prime \prime} \mathrm{S}$, $110^{\circ} 08^{\prime} 12^{\prime \prime}$ E; $687 \mathrm{~m}$ a.s.1.; 8 Jan. 2013; A. Nurinsiyah leg.; measurements: $\mathrm{D}=13.4 \mathrm{~mm}, \mathrm{H}=8.6 \mathrm{~mm}$; MZB 19201.

\section{Paratypes}

INDONESIA - Central Java, Yogyakarta 1 spec.; Kulonprogo, Sibolong, agroforestry along the way to Sibolong Hill; $7^{\circ} 45^{\prime} 00^{\prime \prime}$ S, $110^{\circ} 07^{\prime} 00^{\prime \prime}$ E; $721 \mathrm{~m}$ a.s.1.; ZMH $133441 \cdot 2$ spec.; same data as for preceding; ZMH 133442 - 1 spec.; same data as for preceding; ZMH $133443 \cdot 12$ spec. (det. anat.); Kulonprogo, Sibolong, agroforestry; $7^{\circ} 44^{\prime} 44^{\prime \prime}$ S, $110^{\circ} 08^{\prime} 02^{\prime \prime}$ E; 716 m a.s.1.; MZB 17686 • 9 spec.; same data as for preceding; MZB 17702 - 5 spec.; same data as for preceding; ZMH $133311 \cdot 1$ spec. (det. anat.); Kulonprogo, Sibolong, snakefruit plantation; $7^{\circ} 44^{\prime} 44^{\prime \prime}$ S, $110^{\circ} 08^{\prime} 02^{\prime \prime}$ E; $716 \mathrm{~m}$ a.s.1.; MZB $17724 \cdot 1 \mathrm{spec}$.; same data as for preceding; MZB $17725 \bullet 2$ spec. (det. anat.); Kulonprogo, Sibolong, near Kiskendo Cave, shrubs; $7^{\circ} 44^{\prime} 50^{\prime \prime}$ S, $110^{\circ} 08^{\prime} 09^{\prime \prime}$ E; $721 \mathrm{~m}$ a.s.1.; MZB $17644 \bullet 1 \mathrm{spec}$.; same data as for preceding; ZMH $133444 \bullet 6$ spec.; same data as for holotype; MZB $19202 \cdot 3 \mathrm{spec}$.; same data as for holotype; ZMH $133439 \bullet 1 \mathrm{spec}$.; same data as for holotype; ZMH $133440 \bullet 2 \mathrm{spec}$.; Kulonprogo, Sibolong, agroforestry; $7^{\circ} 44^{\prime} 51^{\prime \prime} \mathrm{S}, 110^{\circ} 08^{\prime} 12^{\prime \prime} \mathrm{E} ; 673 \mathrm{~m}$ a.s.1.; MZB $18039 \bullet 1 \mathrm{spec}$; same data as for preceding; ZMH 133445 • 2 spec.; Kulonprogo, Jonggrangan; 7 44'20" S, $110^{\circ} 08^{\prime} 32^{\prime \prime}$ E; 730 m a.s.1.; ZMH 140034.

\section{Description}

SHELl (Fig. 88; Tables 3-4). Slightly depressed conical-globose, with 5.25-5.75 convex whorls; protoconch smooth; teleoconch with fine, irregular wrinkles; apical side with indistinct incised spiral lines, umbilical side with more distinct spiral lines; without scaly processes; whitish with a distinct brown band above periphery; body whorl rounded; aperture almost circular; upper insertion of peristome distinctly descending; peristome expanded, reflexed and thickened; umbilicus slightly eccentric, moderately narrow, comprising $16-24 \%$ of shell diameter, hardly obscured by columellar edge. 
Genitalia (Figs 47, 71; Table 4). Atrium short; penis short, distal part cylindrical, proximal part slightly dilated, without penis coecum; membranaceous sheath around penis connected with proximal part of epiphallus by tissue; penial retractor runs from diaphragm to distal part of short epiphallus; flagellum long, broadest in its middle part, almost smooth, without node, tapering towards its proximal end; vas deferens narrow and long; vagina long; oviduct short; peduncle of bursa copulatrix subdivided into a short, broad distal part and a narrow and long proximal part, ending in an elongate oval bursa beside albumen gland. Right ommatophoral retractor runs between penis and vagina.

\section{Remarks}

Landouria menorehensis sp. nov. shares with L. pakidulan sp. nov. and L. ciliocincta the whitish shell with a brown band at the periphery. For differences, see remarks under L. pakidulan sp. nov.

\section{Distribution}

Landouria menorehensis sp. nov. is endemic to the Menoreh karst area (Fig. 55).

\section{Discussion}

This revision of the species of Landouria from Java and adjacent islands based on newly collected specimens as well as museum material, revealed that this group represents the most diverse land snail radiation on Java. Only six species of Landouria were recognized from Java in the last revision of the genus based on shell characters by van Benthem Jutting (1950). Since then, only one additional species from Java has been described (Dharma 2015). Our investigation considering also the genitalia as well as DNA sequences recognized twenty-seven species of Landouria from Java and adjacent islands based on the available material. The p-distances between the 16S rDNA sequences of different species of Landouria from Java and Madura Island varied between 2.6\% and 31.0\% (Table 2). Especially the upper limit of variation is high in comparison with the variation of interspecific distances between 16S rDNA sequences in some other helicoid radiations, e.g., 0.9-18.7\% in Mandarina Pilsbry, 1894 (Camaenidae; note that these are corrected distances; uncorrected p-distances would even be lower; Chiba 1999), 4.0-12.9\% in Kimberleytrachia Köhler, 2011 (Camaenidae; p-distances; Criscione \& Köhler 2014), 0.8-19.5\% in Xerocrassa Monterosato, 1892 (Geomitridae; p-distances; Sauer \& Hausdorf 2012), 1.4-33.5\% in Leptaxis Lowe, 1852 (Hygromiidae; corrected distances; van Riel et al. 2005), and 10.7\%-15.9\% in Fruticocampylaea Kobelt, 1871 (Hygromiidae; p-distances; Walther et al. 2016). The high distances between the morphologically delimited species from Java (Table 2) support the conclusion that these populations really represent distinct species, even though some differ only in a few characters of the genitalia.

Most of these species are endemic to small areas within Java. Some of the species that have been described from Java have also been recorded from other islands of the Indo-Australian Archipelago, the Philippines and from Thailand (Martens 1867; Rensch 1932; van Benthem Jutting 1941, 1950, 1959; Vermeulen \& Whitten 1998; Heryanto 2013; Boonngam et al. 2008). It is possible that Landouria winteriana actually also occurs in Sumatra, but most of the other records are probably misidentifications as a result of the similarity of the shells. Thus, a comprehensive revision of the genus in the IndoAustralian Archipelago is necessary.

In Java, the highest diversity of species of Landouria (19 species) can be found in the lowlands (below $500 \mathrm{~m}$ a.s.1.). The diversity decreases with increasing altitude. Only five species occur at altitudes above $1000 \mathrm{~m}$ a.s.1., and only L. monticola and L. davini are known from altitudes above $2000 \mathrm{~m}$ a.s.1. Most species of Landouria occur either in karst areas or on volcanoes. Seventeen species are restricted to karst areas and seven are restricted to volcanoes. Only three species, L. winteriana, L. epiplatia and 
L. ciliocincta, were found in karst areas as well as on volcanoes. Nine species have been recorded from western Java (Banten and West Java), 14 from the central part of Java (Yogyakarta and Central Java) and only six species from East Java. Two species were found both in western and central Java.

Most species are allopatric, but some have overlapping ranges. There are several syntopic occurrences of two species. Most species live in the litter layer in rain forests and do not show special microhabitat preferences. One exception is L. menorehensis sp. nov., which has an arboreal life style. This suggests that the radiation is predominantly non-adaptive, like that of several other land snail groups (Gittenberger 1991; Cameron et al. 1996; Hausdorf 2000; Parmakelis et al. 2005; Sauer \& Hausdorf 2009; Jordaens et al. 2009).

From large areas of Java, we had only shells which could not be accurately identified, or no samples at all. Thus, we suppose that the actual number of species is much higher than the one recorded here. We consider it not unlikely that there are more than 50 species of Landouria in Java.

\section{Acknowledgements}

We are grateful to J. Abbas (Singapore), J. Ablett (NHM), Alfiah (MZB), B. Dharma (Jakarta), J. Goud (RMNH), N.R. Isnaningsih (MZB), R. Janssen (SMF), M. Krogmann (Geosciences Collection of the University of Bremen), F. Köhler (Sydney), J. Lehmann (Geosciences Collection of the University of Bremen), R.M. Marwoto (MZB), E. Neubert (Bern), B. Páll-Gergely (Budapest), R. Prihandini (MZB), B. van der Bijl (RMNH), T. von Rintelen (ZMB) and C. Zorn (ZMB) for providing material, photos or sequences, to T. Setiadi (Jakarta) for preparing the distribution maps, and to A.F. Bakti (Bandung), H. Cahyono (Malang), I. Faizah (Lebak), H. Fauzia (Malang), F. Irsyad (Bandung), Kaspo (Yogyakarta), H. Mada (Bandung), R. Nasrullah (Tasikmalaya), I.E. Nurlela (Bandung), Y. Prasetio (Kabandungan), M. Rizky (Tasikmalaya) and F. Sari (Bandung) for their help during field and laboratory work. We thank E. Neubert (Bern) and B. Páll-Gergely (Budapest) for comments that helped to improve the manuscript. The study is supported by the Indonesian-German Scholarship Program (KEMENDIKTIRISTEK-RI and DAAD), the University of Hamburg (Merit Scholarship Award), the Conchologist of America (Walter Sage Memorial Award 2014) and the Indobiosys Project funded by the German Federal Ministry of Education and Research (project no. 16GW0111K; Museum für Naturkunde, Berlin). Furthermore, this work benefitted from the sharing of expertise within the DFG priority program SPP 1991 TaxonOmics and support from DFG HA 2763/6-1.

\section{References}

Boettger O. 1890. Ad. Strubell's Konchylien aus Java I. Bericht über die senckenbergische naturforschende Gesellschaft 1890: 137-173, pls 5-6.

Boonngam P., Dumrongrojwattana P. \& Matchacheep S. 2008. The diversity of land snail fauna in Chonburi Province, Eastern Thailand. Kasetsart Journal (Natural Science) 42: 256-263.

Bouchet P., Rocroi J.-P., Hausdorf B., Kaim A., Kano Y., Nützel A., Parkhaev P., Schrödl M. \& Strong E.E. 2017. Revised classification, nomenclator and typification of gastropod and monoplacophoran families. Malacologia 61: 1-526. https://doi.org/10.4002/040.061.0201

Cameron R.A.D., Cook L.M. \& Hallows J.D. 1996. Land snails on Porto Santo: adaptive and nonadaptive radiation. Philosophical Transactions of the Royal Society B 351: 309-327.

https://doi.org/10.1098/rstb.1996.0025

Chiba S. 1999. Accelerated evolution of land snails Mandarina in the oceanic Bonin Islands: evidence from mitochondrial DNA sequences. Evolution 53: 460-471.

https://doi.org/10.1111/j.1558-5646.1999.tb03781.x 
Coan E.V. \& Kabat A.R. 2017. 2,400 Years of Malacology (14 ${ }^{\text {th }}$ ed.).

Available from http://www.malacological.org/2004_malacology.html [accessed 28 Feb. 2018].

Criscione F. \& Köhler F. 2014. Molecular phylogenetics and comparative anatomy of Kimberleytrachia Köhler, 2011 - a genus of land snail endemic to the coastal Kimberley, Western Australia with description of new taxa (Gastropoda, Camaenidae). Contributions to Zoology 83: 245-267.

Available from http://www.ctoz.nl/cgi/t/text/get-pdf?c=ctz;idno=8304a03 [accessed 24 Feb. 2019].

Dance S.P. 1986. A History of Shell Collecting. Brill, Leiden.

Davison A. \& Chiba S. 2006. Labile ecotypes accompany rapid cladogenesis in an adaptive radiation of Mandarina (Bradybaenidae) land snails. Biological Journal of the Linnean Society 88: 269-282. https://doi.org/10.1111/j.1095-8312.2006.00624.x

Dharma B. 2015. A new species of Landouria Godwin-Austen 1918 from Jawa, Indonesia (Gastropoda, Bradybaenidae). Schriften zur Malakozoologie 28: 7-12.

Gittenberger E. 1991. What about non-adaptive radiation? Biological Journal of the Linnean Society 43: 263-272. https://doi.org/10.1111/j.1095-8312.1991.tb00598.x

Godwin-Austen H.H. 1918. Zoological results of the Abor Expedition, 1911-12, Mollusca, IX. Records of the Indian Museum 8: 601-621.

Gude G.K. 1905. Description of nine new species of helicoid land shells. Journal of Malacology 12: 11-14, pls 3-4.

Hausdorf B. 2000. The genus Monacha in Turkey (Gastropoda: Pulmonata: Hygromiidae). Archiv für Molluskenkunde 128: 61-151.

Hausdorf B. 2019. Beyond Wallace's line - dispersal of Oriental and Australo-Papuan land-snails across the Indo-Australian Archipelago. Zoological Journal of the Linnean Society 185: 66-76.

https://doi.org/10.1093/zoolinnean/zly031

Hayashi M. \& Chiba S. 2000. Intraspecific diversity of mitochondrial DNA in the land snail Euhadra peliomphala (Bradybaenidae). Biological Journal of the Linnean Society 70: 391-401.

https://doi.org/10.1111/j.1095-8312.2000.tb01230.x

Heryanto. 2013. Keanekaragaman dan kepadatan Gastropoda terestrial di perkebunan Bogorejo kecamatan Gedongtataan kabupaten Pesawaran provinsi Lampung. Zoo Indonesia 22: 23-29.

Hirano T., Kameda Y., Kimura K. \& Chiba S. 2014. Substantial incongruence among the morphology, taxonomy, and molecular phylogeny of the land snails Aegista, Landouria, Trishoplita, and Pseudobuliminus (Pulmonata: Bradybaenidae) occurring in East Asia. Molecular Phylogenetics and Evolution 70: 171-181. https://doi.org/10.1016/j.ympev.2013.09.020

International Commission on Zoological Nomenclature. 1999. International Code of Zoological Nomenclature ( $4^{\text {th }}$ ed.). International Trust for Zoological Nomenclature, London. https://doi.org/10.5962/bhl.title.50608

Jordaens K., Van Riel P., Frias Martins A.M. \& Backeljau T. 2009. Speciation on the Azores islands: congruent patterns in shell morphology, genital anatomy, and molecular markers in endemic land snails (Gastropoda, Leptaxinae). Biological Journal of the Linnean Society 97: 166-176. https://doi.org/10.1111/j.1095-8312.2008.01197.x

Katoh K., Rozewicki J. \& Yamada K.D. 2017. MAFFT online service: multiple sequence alignment, interactive sequence choice and visualization. Briefings in Bioinformatics 2017: 1-7.

https://doi.org/10.1093/bib/bbx108 
Kerney M.P. \& Cameron R.A.D. 1979. A Field Guide to the Land Snails of Britain and North-West Europe. Collins, London.

Köhler F., Shea M. \& Kessner V. In press. Two new species of Landouria Godwin-Austen, 1918 from Timor-Leste (Stylommatophora, Camaenidae). Molluscan Research.

https://doi.org/10.1080/13235818.2018.1557780

Lanfear R., Frandsen P.B., Wright A.M., Senfeld T. \& Calcott B. 2016. PartitionFinder 2: new methods for selecting partitioned models of evolution for molecular and morphological phylogenetic analyses. Molecular Biology and Evolution 34: 772-773. https://doi.org/10.1093/molbev/msw260

Leschke M. 1914. Zur Molluskenfauna von Java und Celebes. Mitteilungen aus dem naturhistorischen Museum in Hamburg 31: 205-284, 1 pl.

Martens E. von. 1867. Die Landschnecken. In: Die Preussische Expedition nach Ost-Asien. Nach amtlichen Quellen. Zoologischer Theil. Vol. 2. Decker, Berlin. https://doi.org/10.5962/bhl.title.12726

Martens E. von. 1892. Landschnecken des Indischen Archipels. In: Weber M. (ed.) Zoologische Ergebnisse einer Reise in Niederländisch Ost-Indien. Vol. 2: 209-264, pls 12-14. Brill, Leiden. https://doi.org/10.5962/bhl.title.52289

Möllendorff O.F. von. 1897. Neue Landschnecken von Java. Nachrichtsblatt der deutschen malakozoologischen Gesellschaft 29: 57-72, 89-97.

Mousson A. 1848. Ueber die Land- und Süsswassermollusken von Java. Mittheilungen der naturforschenden Gesellschaft in Zürich 1: 264-273.

Mousson A. 1849. Die Land- und Süsswasser-Mollusken von Java. Nach den Sendungen des Herrn Seminardirektors Zollinger zusammengestellt und beschrieben. F. Schulthess, Zürich.

https://doi.org/10.5962/bhl.title.14346

Mousson A. 1850. Nachträgliche Bemerkungen zu meinen "Land- und Süsswassermollusken von Java”. Zeitschrift für Malakozoologie 6: 177-186.

Mousson A. 1856. Verzeichniss der von Herrn Dr. Schläfli eingekommenen zweiten malacologischen Sendung. Vierteljahrsschrift der Naturforschenden Gesellschaft in Zürich 1: 395-399.

Neiber M.T. \& Hausdorf B. 2015. Phylogeography of the land snail genus Circassina (Gastropoda: Hygromiidae) implies multiple Pleistocene refugia in the western Caucasus region. Molecular Phylogenetics and Evolution 93: 129-142. https://doi.org/10.1016/j.ympev.2015.07.012

Neiber M.T., Razkin O. \& Hausdorf B. 2017. Molecular phylogeny and biogeography of the land snail family Hygromiidae (Gastropoda: Helicoidea). Molecular Phylogenetics and Evolution 111: 169-184. https://doi.org/10.1016/j.ympev.2017.04.002

Neubert E. 1998. Annotated checklist of the terrestrial and freshwater molluscs of the Arabian Peninsula with descriptions of new species. Fauna of Arabia 17: 333-461.

Neubert E. 2014. Revision of Helix Linnaeus, 1758 in its eastern Mediterranean distribution area, and reassignment of Helix godetiana Kobelt, 1878 to Maltzanella Hesse, 1917 (Gastropoda, Pulmonata, Helicidae). Contributions to Natural History 26: 1-200.

Nurinsiyah A.S. 2015. Land snail fauna of the Sukolilo karst in Java (Indonesia). American Conchologist 43: $30-32$.

Nurinsiyah A.S., Fauzia H., Hennig C. \& Hausdorf B. 2016. Native and introduced land snail species as ecological indicators in different land use types in Java. Ecological Indicators 70: 557-565.

https://doi.org/10.1016/j.ecolind.2016.05.013 
Oostingh C.H. 1923. Recent shells from Java. Part I. - Gastropoda. Mededelingen van de Landbouwhogeschool te Wageningen 26: 1-174, 1 pl.

Páll-Gergely B., Hunyadi A. \& Asami T. 2013. A peculiar new species in the genus Landouria GodwinAusten, 1918 from China (Gastropoda: Heterobranchia: Stylommatophora: Camaenidae). Molluscan Research 33: 130-134. https://doi.org/10.1080/13235818.2013.782794

Parmakelis A., Pfenninger M., Spanos L., Papagiannakis G., Louis C. \& Mylonas M. 2005. Inference of a radiation in Mastus (Gastropoda, Pulmonata, Enidae) on the island of Crete. Evolution 59: 991-1005. https://doi.org/10.1554/04-489

Pfeiffer L. 1842. Symbolae ad Historiam Heliceorum. Sectio alterna. T. Fischer, Cassel [Kassel, Germany]. https://doi.org/10.5962/bhl.title.11903

Pfeiffer L. 1849-1853. Die Schnirkelschnecken nebst den zunächst verwandten Gattungen. Zweiter Theil. In: Küster H.C. (ed.) Systematisches Conchylien-Cabinet Vol. 1 (12-2). Bauer \& Raspe, Nürnberg [Nuremberg]. [See collation in Coan \& Kabat 2017.] https://doi.org/10.5962/bhl.title.50504

Pfeiffer L. 1870-1876. Novitates Conchologicae. Series prima. Mollusca extramarina. Descriptions et Figures de Coquilles extramarines nouvelles. Vol. 4. T. Fischer, Cassel [Kassel, Germany]. [See collation in Coan \& Kabat 2017.] https://doi.org/10.5962/bhl.title.10371

Philippi R.A. 1842-1845. Abbildungen und Beschreibungen neuer oder wenig gekannter Conchylien, unter Mithülfe mehrer deutscher Conchyliologen. Vol. 1. T. Fischer, Cassel [Kassel, Germany]. [See collation in Coan \& Kabat 2017.] https://doi.org/10.5962/bhl.title.10589

Pilsbry H.A. 1893-1895. Helicidae, Vol. 7. Guide to the study of Helices. In: Pilsbry H.A. (ed.) Manual of Conchology. Second Series: Pulmonata. Vol. 9. Academy of Natural Sciences of Philadelphia, Philadelphia. [See collation in Coan \& Kabat 2017.] https://doi.org/10.5962/bhl.title.6534

Rensch B. 1932. Die Molluskenfauna der kleinen Sunda-Inseln Bali, Lombok, Sumbawa, Flores und Sumba. II. (Aus den Ergebnissen der Sunda-Expedition Rensch). Zoologische Jahrbücher, Abteilung für Systematik, Ökologie und Geographie der Tiere 63: 1-130, pls 1-3.

Rensch B. 1934. Landmollusken der Deutschen Limnologischen Sunda-Expedition. Archiv für Hydrobiologie, Supplement 12: 739-758.

Ronquist F., Teslenko M., van der Mark P., Ayres D.L., Darling A., Höhna S., Larget B., Liu L., Suchard M.A. \& Huelsenbeck J.P. 2012. MrBayes 3.2: efficient Bayesian phylogenetic inference and model choice across a large model space. Systematic Biology 61: 539-542.

https://doi.org/10.1093/sysbio/sys029

Sauer J. \& Hausdorf B. 2009. Sexual selection is involved in speciation in a land snail radiation on Crete. Evolution 63: 2535-2546. https://doi.org/10.1111/j.1558-5646.2009.00751.x

Sauer J. \& Hausdorf B. 2012. A comparison of DNA based methods for delimiting species in a Cretan land snail radiation reveals shortcomings of exclusively molecular taxonomy. Cladistics 28: 300-316. https://doi.org/10.1111/j.1096-0031.2011.00382.x

Scheel B.M. \& Hausdorf B. 2012. Survival and differentiation of subspecies of the land snail Charpentieria itala in mountain refuges in the Southern Alps. Molecular Ecology 21: 3794-3808. https://doi.org/10.1111/j.1365-294X.2012.05649.x

Schepman M.M. 1912. On a collection of land and freshwater Mollusca from Java. Proceedings of the Malacological Society of London 10: 229-239, pl. 10.

https://doi.org/10.1093/oxfordjournals.mollus.a063492 
Schepman M.M. 1915. On a collection of land and freshwater and marine Mollusca from Waigeu, Ceram, Pulu Weh and Java. Bijdragen tot de Dierkunde 20: 15-33.

Schileyko A.A. 2004. Treatise on recent terrestrial pulmonate molluscs. Part 12. Bradybaenidae, Monadeniidae, Xanthonychidae, Epiphragmophoridae, Helminthoglyptidae, Elonidae, Humboldtianidae, Sphincterochilidae, Cochlicellidae. Ruthenica Supplement 2: 1627-1764.

Schileyko A.A. 2011. Check-list of land pulmonate molluscs of Vietnam (Gastropoda: Stylommatophora). Ruthenica 21: 1-68.

Schileyko A.A. \& Kuznetsov A.G. 1998. Land snails of the genus Landouria Godwin-Austen, 1918 and some other Bradybaenidae of Nepal (Gastropoda, Pulmonata). Ruthenica 8: 43-54.

Smith B.J. \& Djajasasmita M. 1988. The land molluscs of the Krakatau Islands, Indonesia. Philosophical Transactions of the Royal Society B 322: 379-400. https://doi.org/10.1098/rstb.1988.0133

Sokolov E.P. 2000. An improved method for DNA isolation from mucopolysaccharide-rich molluscan tissues. Journal of Molluscan Studies 66: 573-575. https://doi.org/10.1093/mollus/66.4.573

Sukumaran J. \& Holder M.T. 2010. DendroPy: a Python library for phylogenetic computing. Bioinformatics 26: 1569-1571. https://doi.org/10.1093/bioinformatics/btq228

Tryon G.W. 1887. Helicidae, Vol. I. In: Tryon G.W. (ed.) Manual of Conchology. Second Series: Pulmonata. Vol. 3. Academy of Natural Sciences of Philadelphia, Philadelphia. [See Coan \& Kabat 2017.] https://doi.org/10.5962/bhl.title.6534

van Benthem Jutting W.S.S. 1941. Non-marine Mollusca from the satellite islands surrounding Java. Archives néerlandaises de Zoologie 5: 251-348.

van Benthem Jutting W.S.S. 1950. Systematic studies on the non-marine Mollusca of the Indo-Australian archipelago. II. Critical revision of the Javanese pulmonate land-shells of the families Helicarionidae, Pleurodontidae, Fruticicolidae and Streptaxidae. Treubia 20: 381-505.

van Benthem Jutting W.S.S. 1959. Catalogue of the non-marine Mollusca of Sumatra and of its satellite islands. Beaufortia 7: 41-191, pls 1-2, map.

van Riel P., Jordaens K., van Houtte N., Frias Martins A.M., Verhagen R. \& Backeljau T. 2005. Molecular systematics of the endemic Leptaxini (Gastropoda: Pulmonata) on the Azores islands. Molecular Phylogenetics and Evolution 37: 132-143. https://doi.org/10.1016/j.ympev.2005.03.019

Vermeulen J.J. \& Whitten A.J. 1998. Fauna Malesiana. Guide to the Land Snails of Bali. Backhuys Publishers, Leiden.

Wade C.M., Hudelot C., Davison A., Naggs F. \& Mordan P.B. 2007. Molecular phylogeny of the helicoid land snails (Pulmonata: Stylommatophora: Helicoidea), with special emphasis on the Camaenidae. Journal of Molluscan Studies 73: 411-415. https://doi.org/10.1093/mollus/eym030

Walther F., Neiber M.T. \& Hausdorf B. 2016. Systematic revision and molecular phylogeny of the land snail genus Fruticocampylaea (Gastropoda: Hygromiidae) from the Caucasus region. Systematics and Biodiversity 14: 32-54. https://doi.org/10.1080/14772000.2015.1100691

Wiegmann F. 1893. Beiträge zur Anatomie der Landschnecken des Indischen Archipels. In: Weber M. (ed.) Zoologische Ergebnisse einer Reise in Niederländisch Ost-Indien Vol. 3: 112-259, pls 9-16. Brill, Leiden. https://doi.org/10.5962/bhl.title.52289

Zilch A. 1966. Die Typen und Typoide des Natur-Museums Senckenberg, 35: Mollusca, Camaenidae (5). Archiv für Molluskenkunde 95: 293-319. 
Zilch A. 1967. Aus der Geschichte des Senckenberg-Museum, Nr. 13. Geschichte der malakologischen Sektion. Archiv für Molluskenkunde 97: 7-43.

Zwickl D.J. 2006. Genetic Algorithm Approaches for the Phylogenetic Analysis of Large Biological Sequence Datasets under the Maximum Likelihood Criterion. PhD Thesis, University of Texas at Austin, Austin.

Manuscript received: 27 September 2018

Manuscript accepted: 14 March 2019

Published on: 21 May 2019

Topic editor: Rudy Jocqué

Desk editor: Danny Eibye-Jacobsen

Printed versions of all papers are also deposited in the libraries of the institutes that are members of the EJT consortium: Muséum national d'Histoire naturelle, Paris, France; Meise Botanic Garden, Belgium; Royal Museum for Central Africa, Tervuren, Belgium; Natural History Museum, London, United Kingdom; Royal Belgian Institute of Natural Sciences, Brussels, Belgium; Natural History Museum of Denmark, Copenhagen, Denmark; Naturalis Biodiversity Center, Leiden, the Netherlands; Museo Nacional de Ciencias Naturales-CSIC, Madrid, Spain; Real Jardín Botánico de Madrid CSIC, Spain; Zoological Research Museum Alexander Koenig, Bonn, Germany. 